\title{
ADAPTIVE CRITIC FLIGHT CONTROL FOR A GENERAL AVIATION AIRCRAFT: SIMULATIONS FOR THE BEECH BONANZA FLY-BY-WIRE TESTBED
}

\author{
A Thesis by \\ Rajeev Chandramohan \\ Bachelor of Electronics \& Communication Engineering, Bangalore University, 2001
}

Submitted to the Department of Aerospace Engineering and the faculty of the Graduate School of

Wichita State University

in partial fulfillment of

the requirements for the degree of

Master of Science

July 2007 
(C) Copyright 2007 by Rajeev Chandramohan

All Rights Reserved 


\section{ADAPTIVE CRITIC FLIGHT CONTROL FOR A GENERAL AVIATION AIRCRAFT: SIMULATIONS FOR THE BEECH BONANZA FLY-BY-WIRE TESTBED}

I have examined the final copy of this Thesis for form and content and recommend that it be accepted in partial fulfillment of the requirement for the degree of Master of Science with a major in Aerospace Engineering

James E Steck, Committee Chair

We have read this Thesis

and recommend its acceptance:

Kamran Rokhsaz, Committee Member

John Watkins, Committee Member

Silvia Ferrari, Committee Member 


\section{ACKNOWLEDGMENTS}

I would like to thank my advisor, Dr. James E Steck, for his guidance and support. I would like to extend my gratitude to the members of my committee, Dr. Kamran Rokhsaz, Dr. John Watkins and Dr. Silivia Ferrari, for their helpful comments and suggestions. I also want to thank my family and friends. 


\begin{abstract}
An adaptive and reconfigurable flight control system is developed for a general aviation aircraft. The flight control system consisting of two neural networks is developed using a two phase procedure called the pre-training phase and the online training phase. The adaptive critic method used in this thesis was developed by Ferrari and Stengel. In the pre-training phase the architecture and initial weights of the neural network are determined based on linear control. A set of local gains for the linearized model of the plant is obtained at different design points on the velocity v/s altitude envelope using an LQR method. The pre-training phase guarantees that the neural network controller meets the performance specifications of the linear controllers at the design points. Online training uses a dual heuristic adaptive critic architecture that trains the two networks to meet performance specifications in the presence of nonlinearities and control failures. The control system developed is implemented for a three-degree-of-freedom longitudinal aircraft simulation. As observed from the results the adaptive control system meets performance requirements, specified in terms of the damping ratio of the phugoid and short period modes, in the presence of nonlinearities. The neural network controller also compensates for partial elevator and thrust failures. It is also observed that the neural network controller meets the performance specification for large variations in the parameters of the assumed and actual models.
\end{abstract}




\section{TABLE OF CONTENTS}

Chapter

Page

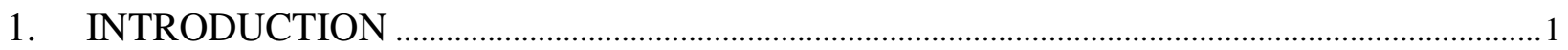

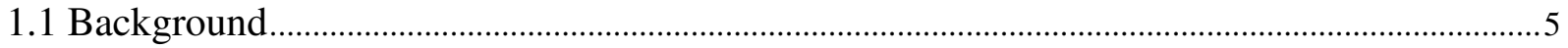

1.2 Artificial neural networks..........................................................................................................

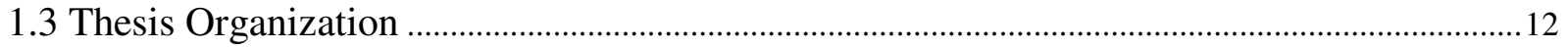

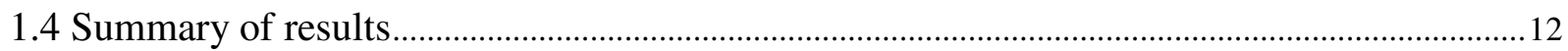

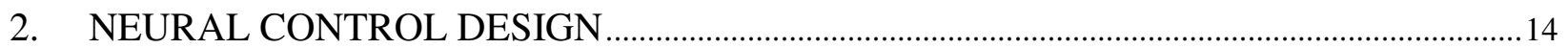

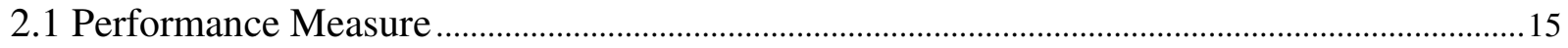

2.2 Aircraft optimal control problem ……………………………………………………………......

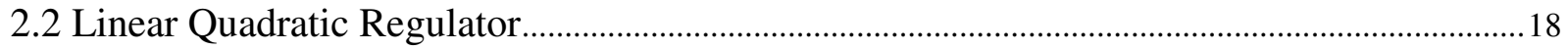

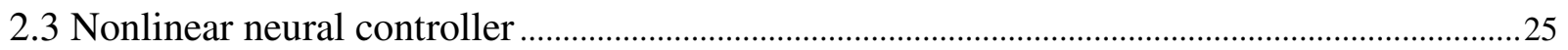

2.4 Adaptive critic fundamentals..........................................................................................................

3. ALGEBRAIC TRAINING OF NEURAL NETWORKS ……………………………………….....

3.1 Exact gradient based solution .......................................................................................................

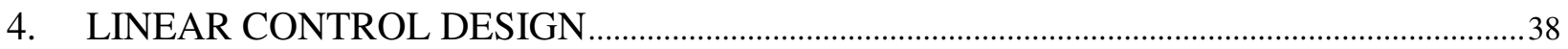

4.1 Flight envelope ………………………………………………………………………………….....

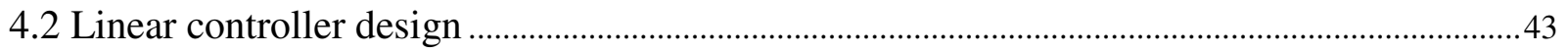

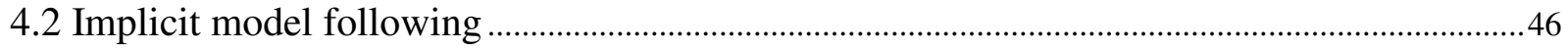

4.3 Neural network based controller .......................................................................................................

4.4 Response of initialized neural network ……………………………………………………....5

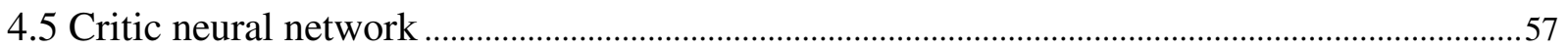




\section{TABLE OF CONTENTS (Cont.)}

Chapter

Page

5. ADAPTIVE CRITIC FLIGHT CONTROL ………………………………………………….......60

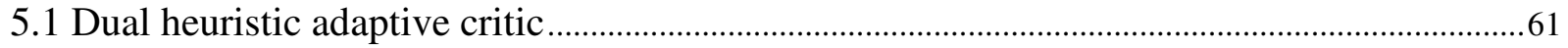

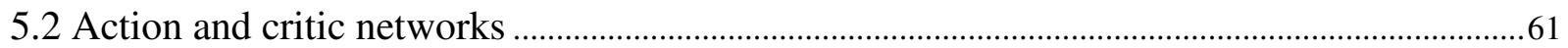

5.3 Online adaptation .........................................................................................................................

6. SIMULATION AND RESULTS ……………………………………………………………ㄱ……

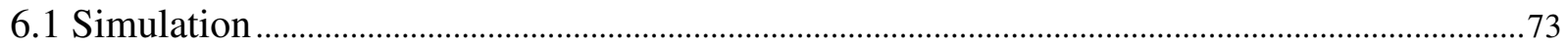

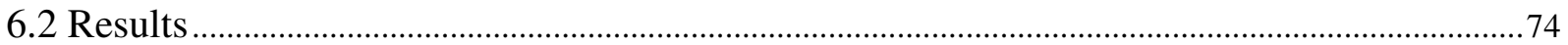

7. CONCLUSION

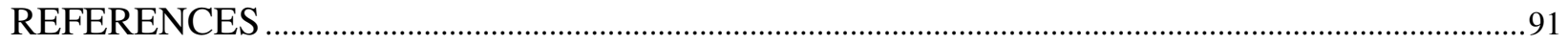




\section{LIST OF FIGURES}

Figure $\quad$ Page

Figure 1: Backward dynamic programming for a two stage process...................................... 7

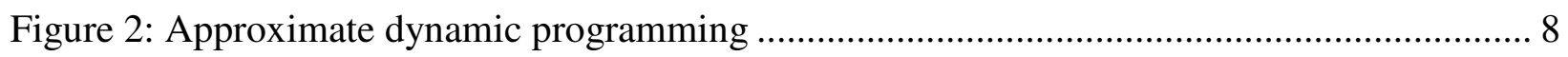

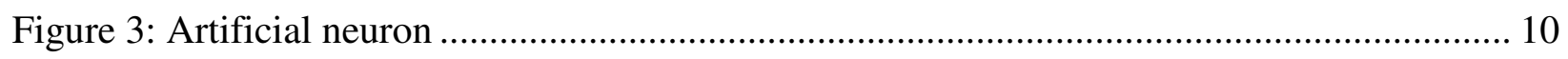

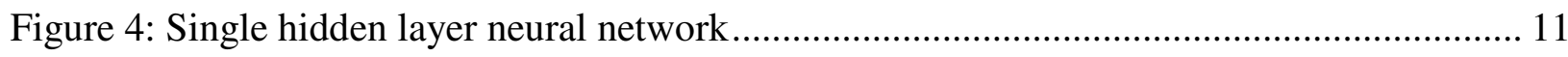

Figure 5: Relationship between Cost function and Value function .................................... 21

Figure 6: Adaptive critic based neural network controller. ................................................ 25

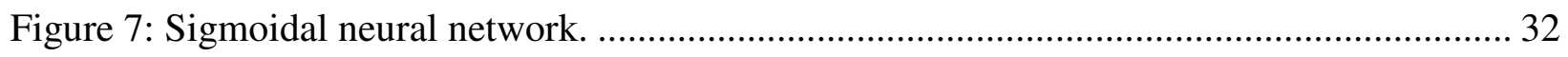

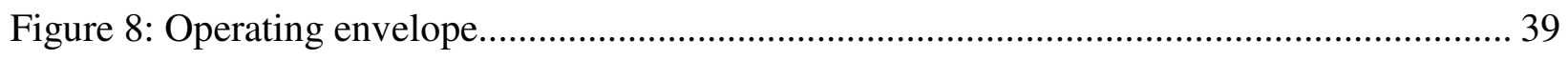

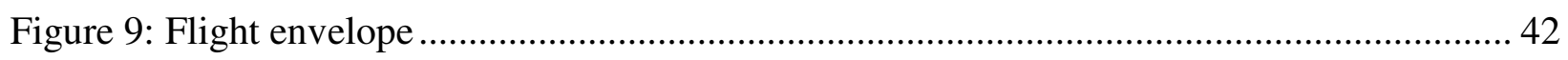

Figure 11: Neural network based PI controller ......................................................... 51

Figure 12: Initialized feedback neural network architecture ............................................. 54

Figure 13: Initialized integral neural network architecture............................................ 55

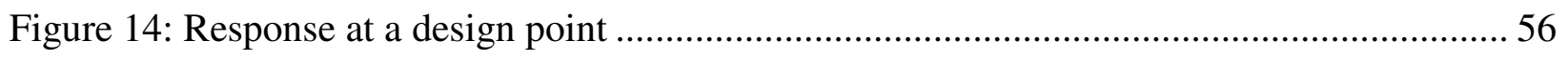

Figure 15: Response at an interpolation point ......................................................... 57

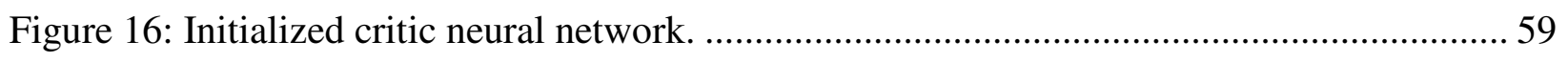

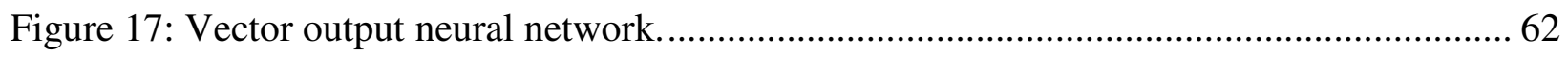

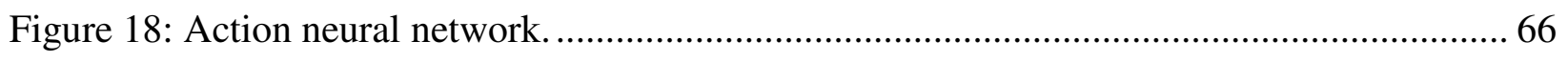

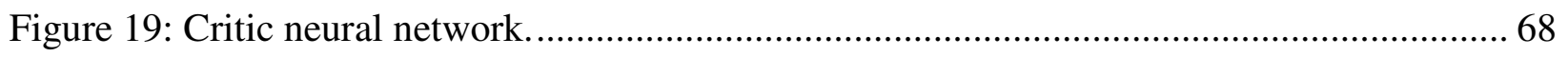

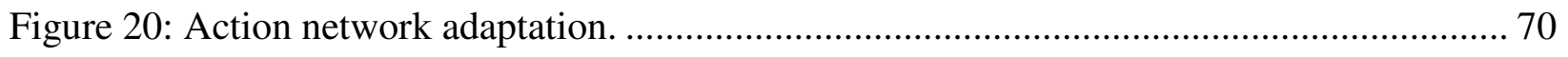

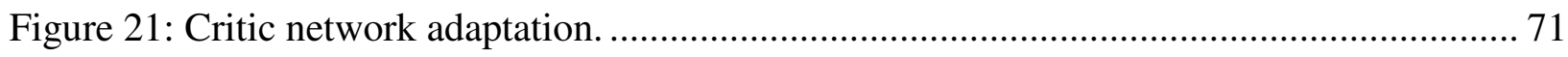

Figure 22: Velocity response and throttle setting for partial elevator failure .......................... 76 


\section{LIST OF FIGURES (Cont.)}

$\begin{array}{lll}\text { Figure } & \text { Page }\end{array}$

Figure 23: Flight path angle response and elevator deflection for partial elevator failure.......... 76

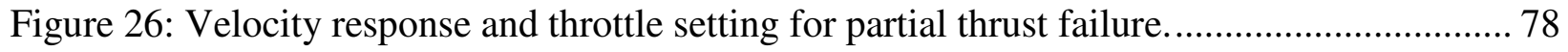

Figure 27: Flight path angle response and elevator deflection for partial thrust failure............. 78

Figure 28: Angle of attack and pitch angle for partial thrust failure. .................................... 79

Figure 29: Incremental cost for partial thrust failure ..................................................... 79

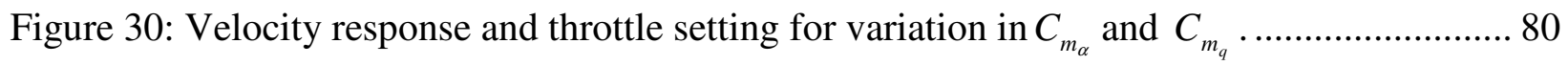

Figure 31: Flight path angle response and elevator deflection for variation in $C_{m_{\alpha}}$ and $C_{m_{q}} \ldots \ldots .81$

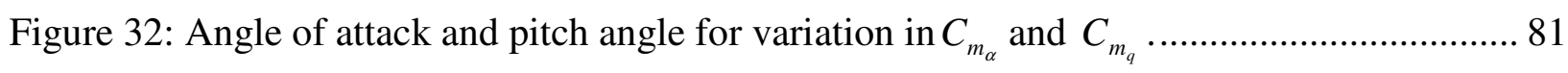

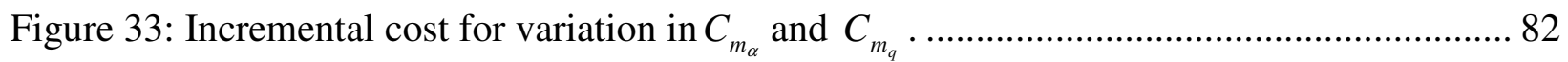

Figure 34: Flight path angle response and elevator deflection for variation in $C_{D_{k}} \ldots \ldots \ldots \ldots \ldots \ldots . . . . . . . .13$

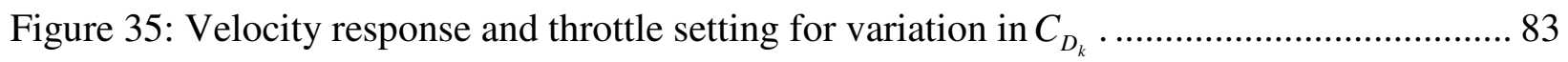

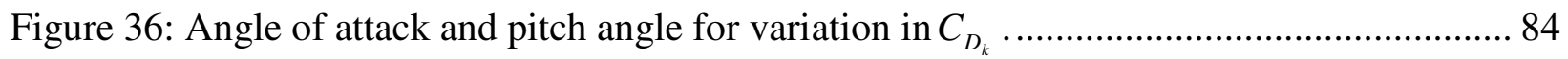

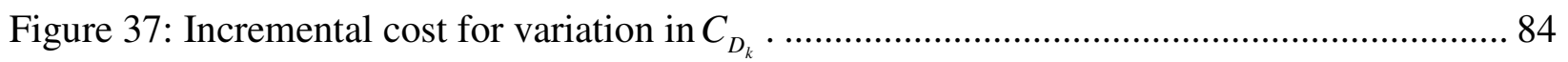

Figure 38: Velocity response and throttle setting for a command of $-40 \mathrm{ft} / \mathrm{s}$ in velocity. ........... 85

Figure 39: Flight path angle response and elevator deflection for a command of $-40 \mathrm{ft} / \mathrm{s}$ in

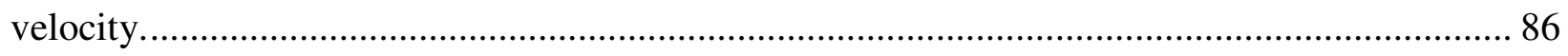

Figure 40: Angle of attack and theta time history for a command of $-40 \mathrm{ft} / \mathrm{s}$ in velocity........... 86

Figure 41: Altitude time history for a command of $-40 \mathrm{ft} / \mathrm{s}$ in velocity .................................. 87

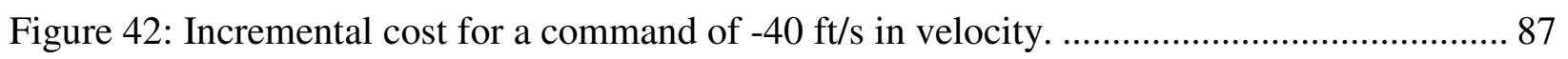




\section{LIST OF TABLES}

Table $\quad$ Page

Table 1: Flying qualities for class I aircraft in terminal phase flight ....................................46 


\section{LIST OF SYMBOLS}

$a$

$A R$

$b$

$C$

$C_{D_{0}}$

$C_{B}$

$C_{F}$

$C_{I} \quad$ Integral gain matrix

$C_{L} \quad$ Lift coefficient

$D$

$e$

$F$

$G$

$h$

$H_{x} \quad$ Jacobian matrix of a linear systems output with respect to the state

$H_{u} \quad$ Jacobian matrix of a linear systems output with respect to control

$H[\bullet] \quad$ Hamiltonian

$J$

$J^{*}$

$L[\bullet]$

Neural network input bias

Total aircraft drag

Oswalds efficiency factor

Linear state Jacobian matrix

Linear control Jacobian matrix

Aircraft altitude

Cost function

Optimal cost

Lagrangian 


\section{LIST OF SYMBOLS (Cont.)}

$M \quad$ Matrix of cross-coupling weighting between the state and control

$N N_{A} \quad$ Action neural network

$N N_{C} \quad$ Critic neural network

$p \quad$ Neural network input vector

$P \quad$ Steady state Riccati matrix

$P_{A} \quad$ Power available

$P_{R} \quad$ Power required

$q \quad$ Aircraft pitch rate

$Q \quad$ State weighting matrix

$R \quad$ Control weighting matrix

$S \quad$ Matrix of sigmoidal functions evaluated at input to node values

$S \quad$ Aircraft wing reference area

$t \quad$ Time variable in continuous-time representation

$t_{k} \quad$ Time variable in sampled-time representation

$T_{A} \quad$ Thrust available

$T_{R} \quad$ Thrust required

$u \quad$ Control signal

$u_{0} \quad$ Trim control signal

$\tilde{u} \quad$ Control deviation from commanded control

$v \quad$ Neural network output weight vector

V Aircraft velocity 


\section{LIST OF SYMBOLS (Cont.)}

\begin{tabular}{|c|c|}
\hline$V[\bullet]$ & Cost-to-go or value function \\
\hline$V_{\infty}$ & Free stream velocity \\
\hline$W$ & Neural network input weight matrix \\
\hline$W$ & Aircraft weight \\
\hline$x$ & State variable \\
\hline$x_{a}$ & Augmented state \\
\hline$y_{c}$ & Command input \\
\hline$y$ & Output state \\
\hline$z$ & Output of neural network \\
\hline$\alpha$ & Angle of attack \\
\hline$\delta_{T}$ & Throttle position \\
\hline$\delta_{e}$ & Elevator deflection \\
\hline$\Delta()$ & Perturbation from the equilibrium value \\
\hline$\gamma$ & Vertical flight path angle \\
\hline$\lambda$ & Adjoint vector \\
\hline$\theta$ & Aircraft pitch angle \\
\hline$\rho_{\infty}$ & Free stream air density \\
\hline$\sigma$ & Sigmoid activation function \\
\hline$\xi$ & Time integral of output error \\
\hline
\end{tabular}




\section{INTRODUCTION}

Controllers can be designed for systems so as to change the response of the system to meet performance specifications. Linear control theory provides a basis for designing control systems to meet performance specifications. One of the major disadvantages of linear control theory is that the system to be controlled must be modeled by linear ordinary differential equations. Most systems in practice are highly nonlinear in nature. This renders linear control system design unsuitable for many practical systems. To overcome this drawback, the systems are linearized about an operating point and control systems are designed at the operating point using linear control theory. The control law developed about an operating point deteriorates in performance for conditions further away from that point due to nonlinearities. This necessitates the design of a controller that meets performance specifications over its entire operating range.

A fixed controller that meets performance specifications in the presence of differences between the actual plant and the assumed model is termed to be robust. A controller that varies its parameters during its operation is termed to be adaptive or gain scheduled. An adaptive controller can be expected to perform better in the presence of uncertainty than a fixed controller. A controller that can meet performance specifications in the presence of system failure is said to be reconfigurable.

The objective of this thesis was to develop an adaptive control system that is both robust and reconfigurable for a general aviation aircraft that is modeled by nonlinear ordinary differential equations. The adaptive controller is developed using neural networks in conjunction with linear

control theory. The proposed control system would render the aircraft easier to fly, even for pilots with relatively low piloting skills. The controller adapts and reduces pilot workload in the presence of partial control failures making it safer to fly the aircraft. 
The application of neural networks for the control of nonlinear systems has been studied extensively in the past by various authors. Several different schemes using neural networks for control have been investigated, these include the inverse controller architecture using neural networks, dynamic inversion neural network control and adaptive critic based neural network control. In addition the areas of application of neural networks for control studied include robotics, chemical plant control, and vehicle control among others. Neural network control of aircraft have also been studied extensively in the past using different control architectures.

Suresh et al. [1] have developed a neural network (NN) based control system to improve damping and follow piloted input commands for the F-8 fighter aircraft. The controller is used to approximate the control law derived using linear system theory. The NN controller is trained offline using a reference model of the aircraft and online the NN adapts for variations in aerodynamic stability derivatives and control derivatives.

Calise et al. [2] have developed a direct adaptive tracking controller based on neural networks. The neural networks were used to represent the nonlinear inverse transformation for feedback linearization. In this method the neural networks were trained offline using a nominal mathematical model of the F-18 high performance aircraft. The neural networks are then trained online to compensate for the modeling errors between the actual and nominal mathematical model.

Rysdyk et al. [3] have used a neural network augmented dynamic model inversion control for a civilian rotorcraft. They use neural networks to account for nonlinearities and modeling error between the actual plant and an inverse controller. One of the main advantages of the scheme proposed by the authors is that there is no need for off-line training as all the information available is incorporated in the design of the inverse controller. The above scheme is 
implemented for attitude hold and attitude command in the longitudinal channel. The proposed system provides consistent response over the entire flight envelope of the tiltrotor including in transition flight.

Perhinschi et al. [4] have used a neural network augmented dynamic inversion model control for the F-15 high performance aircraft. In addition they use a neural network that is trained offline to provide the aerodynamic and control stability derivatives. They have shown that when the offline neural network is adapted online the compensating signal of the neural network used to remove dynamic inversion reduces.

Burlet et al. [5] have developed a neural network based adaptive controller based on linear programming and adaptive critic techniques for a simulated C-17 aircraft. Linear programming techniques have been used to allocate the control deflections generated in the presence of failures in an optimal manner. The adaptive critic is used to update the parameters of the reference model to provide consistent handling qualities. Piloted simulations of the controller were carried out to asses the performance of the controller in the presence of simulated failure conditions.

Steck et al. [6] have implemented a neural network augmented dynamic model inversion control for general aviation aircraft control. They have also investigated the performance of the control scheme in the presence of turbulence [7] as well as microburst [8]. The authors have also presented pilot evaluation of the controller using flight simulation. The control scheme developed was shown to be able to follow piloted commands even in the presence of unanticipated failures in the system.

Kulkarni et al. [9] have developed a neural network based methodology for the design of optimal controllers for nonlinear system. The system consists of two neural networks: one predicts the cost to go from the present state; the second neural network is trained to minimize 
the output of the first neural network. Since the second network minimizes the output of the first neural network it converges to the optimal solution and can be used as an optimal controller.

Balakrishnan et al. [10] have studied extensively the application of adaptive critic for aircraft optimal control. The adaptive critic controller is evaluated for the longitudinal control of an aircraft and is seen to provide near optimal control policies for the control of the aircraft. In another work Balakrishnan et al. [11] have designed an adaptive critic based autolanding controller for aircraft. The performance of the adaptive critic based controller is compared with that of a classical PID controller.

Shin et al. [12] have demonstrated the use of a neural network based adaptive output feedback control for an F-15 aircraft at high angles of attack. The method utilizes a fixed gain linear controller in combination with a neural network to compensate for model inversion error. The linear controller is designed using output feedback linearization. The total control input to the aircraft is the sum of the linear controller output and the output of the neural network which compensates for the modeling error between the actual and assumed dynamics at high angles of attack.

Williams-Hayes [13] with the intelligent flight control system project team has developed a neural network based adaptive controller for a highly modified F-15B aircraft. The neural network based adaptive controller is used to optimize aircraft performance and also to compensate for unknown failures during flight. Simulations of the experiments to be performed in the flight test are used to evaluate the performance of the adaptive controller.

Ferrari et al. [14] have proposed a novel scheme for classical neural synthesis for control of non linear systems. In this scheme the initialization of the neural networks is based on linear control theory. The parameters for the neural networks are obtained by designing linear control 
gains for a set of linear models covering the entire operating range of the aircraft using LQR methods. The initialized neural networks are then adapted online using adaptive critic method to improve performance based on actual system dynamics. They have applied this scheme to the design of six degree of freedom business jet simulation. The simulations show that the proposed scheme follows pilot inputs in the presence of parameter variations and unanticipated control failures. This method is used in this thesis to design an adaptive controller for three degree of freedom general aviation aircraft control.

The methodology presented in this thesis combines linear control theory and neural networks to control the nonlinear aircraft dynamics. Gain scheduling is used in the design of the linear controller as it provides a means of applying linear control laws over the entire envelope of the aircraft which is widely used in the industry. The initialized controller designed using LQR method is robust to nonlinearities and model uncertainties. In the online phase the controller adapts to the actual dynamics of the aircraft and accommodates for a larger degree of uncertainty than the initialized controller.

\subsection{Background}

The control of nonlinear systems so as to minimize a cost is one of the fundamental problems in optimal control theory. The cost $J$ is a measure of the performance of the control system. A control policy is said to be optimum if the controller minimizes the cost. The cost is a combination of the physically important states and control signals. There are various kinds of performance measures for different kinds of problems. Some of the different types of costs are minimum time problems, terminal control problems, minimum control effort problems, tracking problems etc. Once the performance measure or cost to be minimized is determined the next task is to determine a control function that minimizes the cost. It is often useful to find the optimal 
control policy as a function of the state at any given time $t$ as this can be used to generate the control based on the state without the need for a lookup table implementation. Two methods exist for finding the optimal policy functional so as to minimize the cost. These are the principle of dynamic programming and the minimum principle of Pontryagin. Adaptive critic designs are based on the principle of dynamic programming.

Dynamic programming uses the principle of optimality to determine the optimal control policy. The principle of optimality can be explained as follows. The optimal solution to a global problem is a combination of optimal solutions to all of its constituent sub problems.

Let us now apply the principle of optimality to a two stage process in order to determine the optimal policy from the initial to the final state. Now if we determine the optimal path from all the intermediate states to the last state, The principle of optimality states that this path lies on the globally optimal path from the initial state passing through the intermediate state. Let the optimal path to the final state $f$ from the intermediate states $c, d, e$ be associated with the optimal $\operatorname{cost} J_{c f}^{*}, J_{d f}^{*}, J_{e f}^{*}$ as shown in the Figure 1 . Now by the principle of optimality, the optimal cost from $b$ to $f$ via each of the intermediate states is given by

$$
J_{b c f}^{*}=J_{b c}+J_{c f}^{*}, J_{b d f}^{*}=J_{b d}+J_{d f}^{*}, J_{b e f}^{*}=J_{b e}+J_{e f}^{*}
$$

The overall optimal path from $b$ to $f$ is found by taking the minimum of the above three values, i.e.

$$
J_{b f}^{*}=\min \left(J_{b c f}^{*}, J_{b d f}^{*}, J_{b e f}^{*}\right)
$$




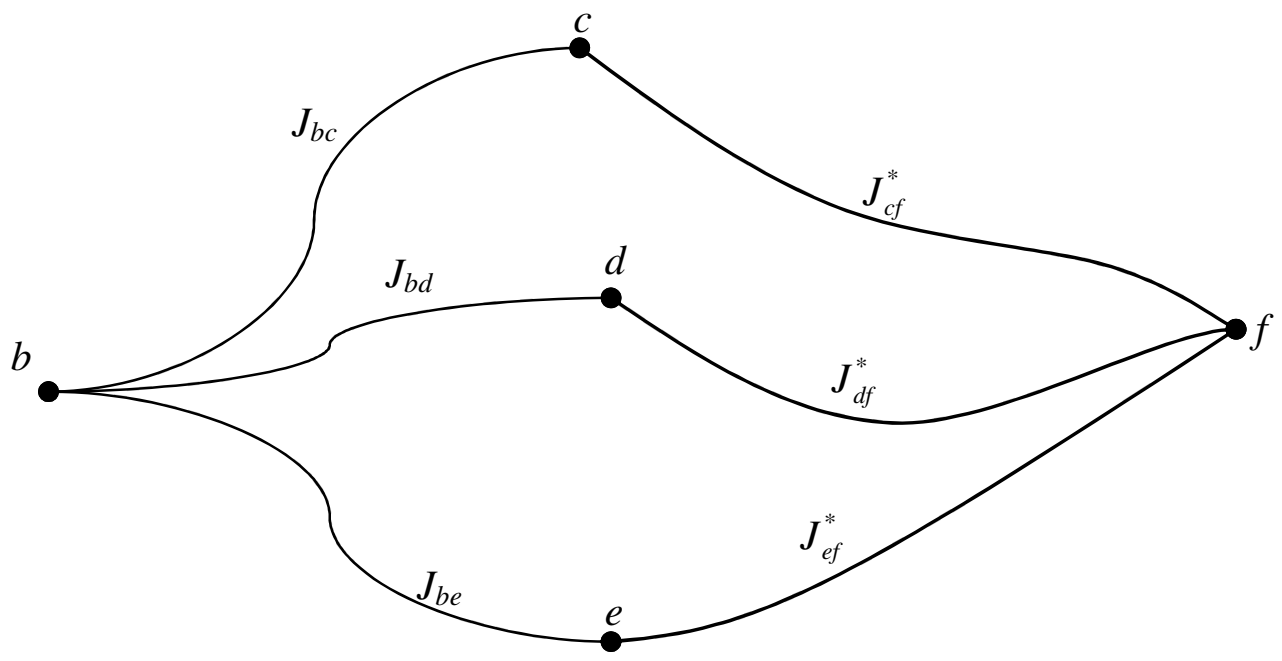

Figure 1: Backward dynamic programming for a two stage process.

Thus we can find the optimal global path by trying all the local paths, then following the optimal paths from then on for each of these and choosing the one with minimum cost. For systems with a large number of states and large number of stages, the above process has to be repeated backward for each stage and the optimal values stored until we reach the initial stage from which we can determine the optimal policy to reach the final value from the initial state. This becomes infeasible for systems with even a relatively few number of states and the number of computations grows exponentially with the number of states; this is commonly referred to as the "curse of dimensionality". The Approximate dynamic programming method uses incremental optimization combined with a neural network to reduce the computations associated with evaluating cost at each stage.

In dynamic programming we progress backward in time starting from the final state where as in approximate dynamic programming [16] we progress forward in time. In approximate dynamic programming the optimal control policy and the cost associated with it is calculated 
based on the current state. This can be explained better by considering a two stage process as shown in Figure 2. At the first stage $a$, the cost associated with going from $a$ to $b, J_{a b}$ can be calculated based on the current state and the current control needed to go from $a$ to $b$. The optimal cost to go from the state $b$ to the final state is estimated as $\hat{J}_{b f}^{*}$ by a parametric structure that approximates the cost to go based on the current state and control. The path $a-b$ is chosen to minimize the cost $J_{a b}+\hat{J}_{b f}^{*}$. At the next stage, $b-c$ the above procedure is repeated but with the information available from the previous stage the policy and cost approximations $\left(\hat{J}^{*}\right)$ have had a chance to improve and the next path from $c-f$ is closer to the optimal trajectory.

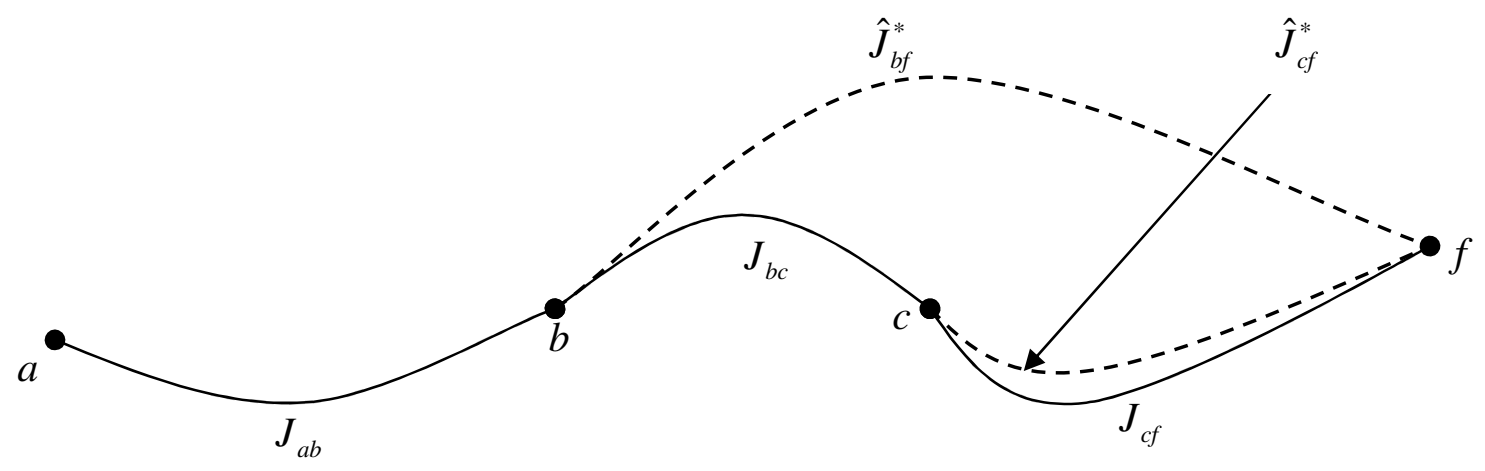

Figure 2: Approximate dynamic programming

Adaptive critic design is based on approximate dynamic programming and produces the most general solution of approximate dynamic programming. The importance of adaptive critic design is that it attempts to overcome the curse of dimensionality by ensuring convergence to the optimal solution over time. There are three different forms of adaptive critic designs [17]. In the 
first form, called heuristic dynamic programming, the neural network is used to approximate the optimal cost to go explicitly and another neural network to approximate the optimal control policy. It has been observed that heuristic dynamic programming converges slowly to the optimal control policy. Dual heuristic dynamic programming [17] also uses two networks one neural network to approximate the optimal control policy and another to approximate the gradient of the cost to go with respect to the state of the system. It has been shown that dual heuristic dynamic programming converges faster to the optimal policy as compared to heuristic dynamic programming. The third form is called globalized dual heuristic programming [17] which also uses two networks. One neural network is used to approximate the optimal control policy and another network is used to approximate both the cost to go value and the gradient of the cost to go value with respect to the state. In this thesis the adaptive critic design used is based on the second method, dual heuristic programming method.

\subsection{Artificial neural networks}

Approximate dynamic programming is based on approximation of the optimal policy function and the value function or its derivatives with respect to the state. This necessitates the use of function approximators in the adaptive critic based control system design. Several parametric structures are presently in use to approximate the nonlinear relationship between the inputs and the outputs. Some examples of parametric structures used for function approximation are splines, wavelets, artificial neural networks etc.

In the adaptive control system design proposed here the parametric structure used for function approximation should possess the ability to learn a nonlinear mapping when a large number of input output training pairs are available as well as when only one input output training pair is available. One other feature of the parametric structure should be that it should be able to 
approximate the nonlinear mapping of multidimensional input output space. Of all the parametric structures which can be used for function approximators only the artificial neural networks have the above properties.

Artificial neural networks are based on the way biological neurons present in the brain process information. Artificial neural networks are composed of a large number of processing elements arranged in the form of layers and connected with each other by a means of communication links. Each of these communication links has a weight associated with it; the weights of these communication links are changed in order to approximate the nonlinear functional relationship between the input and the output. Each neuron also has an internal process, called the activation function that acts on the net input to the neuron. Some of the commonly used activation functions are the sigmoid, tanh, linear, radial basis etc. The basic structure of an artificial neuron is as shown in Figure 3.

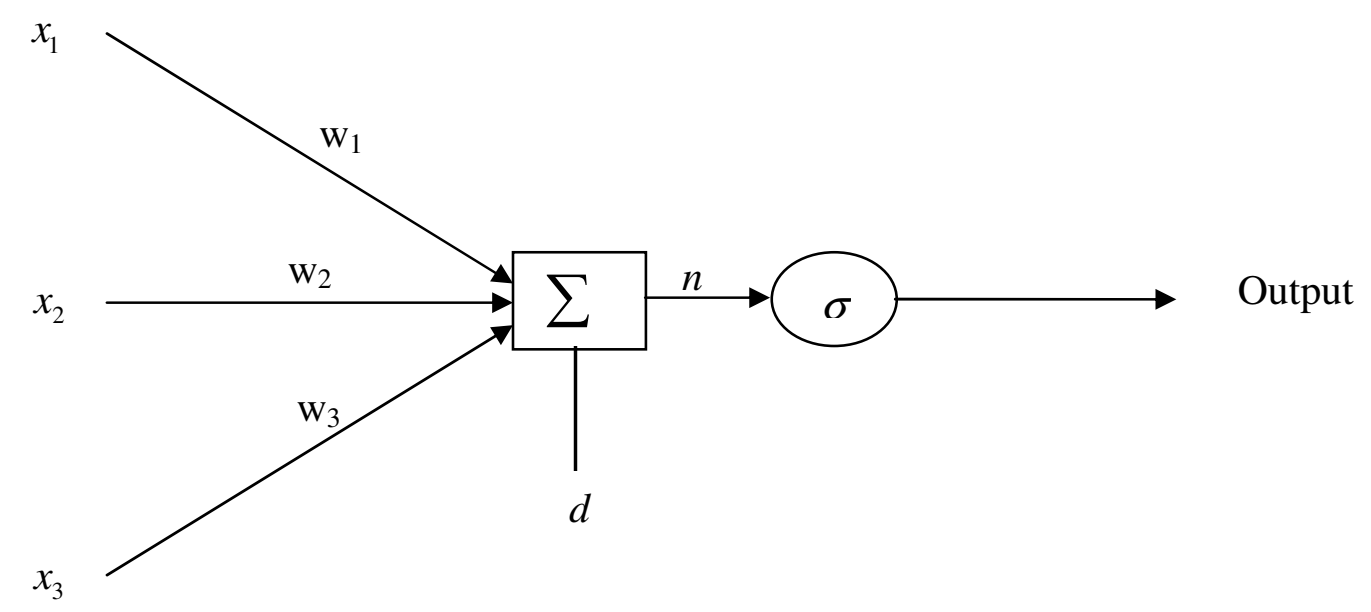

Figure 3: Artificial neuron 
The neural networks used for the adaptive control system in this thesis, use single hidden layer neural networks with sigmoidal activation functions as shown in Figure 4. In most neural network applications the neural networks are trained using supervisory training algorithms. In supervisory training algorithms the goal is to reduce the error between the actual output of the neural network and a target output. Backpropogation algorithms have been devised to modify the weights of the neural network architecture in order to reduce the error. Once the neural networks output is close to the desired output, the training is stopped and the neural network is said to be trained. One of the problems associated with the training of neural networks is the problem of overfitting. Overfitting is characterized by the network having a desired network error for the training set, but having large errors for input output pairs not present in the training set.

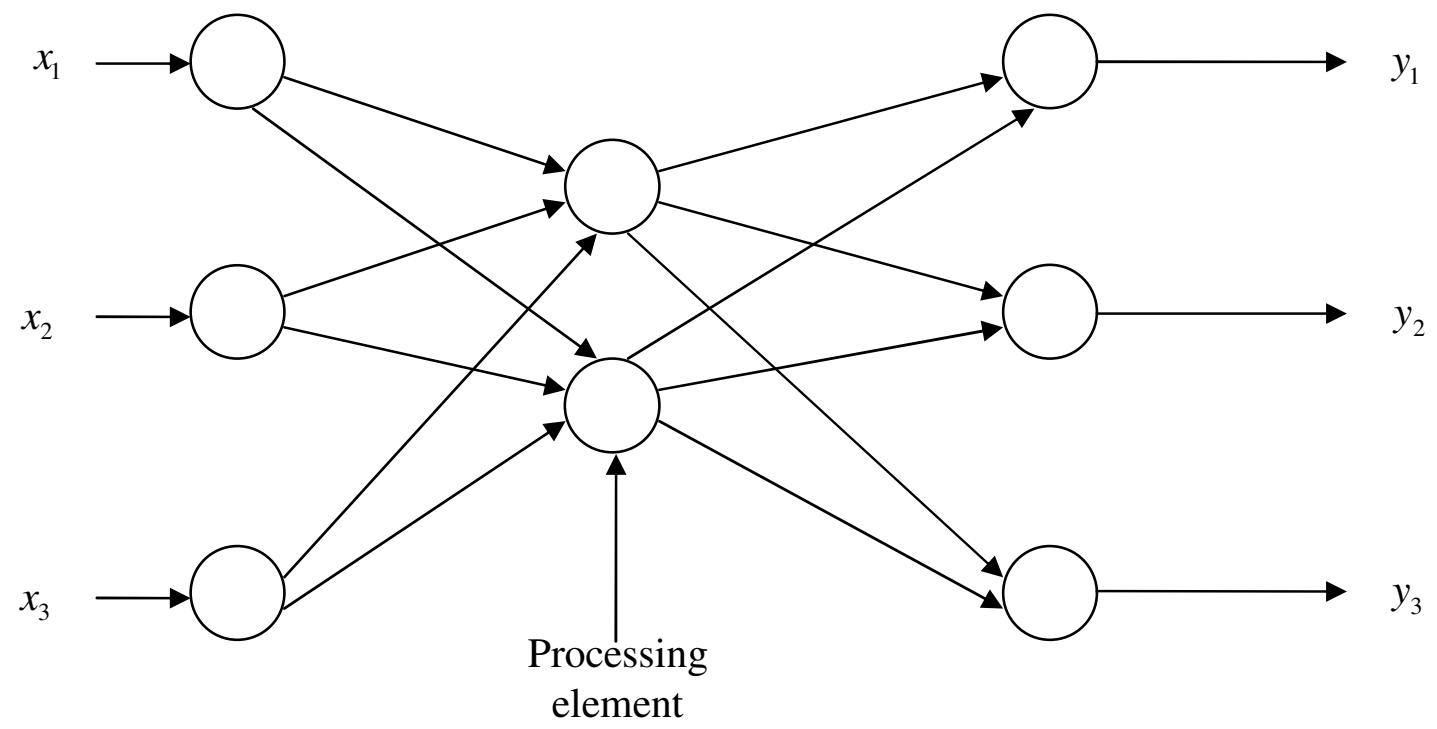

Figure 4: Single hidden layer neural network

In this thesis a backpropogation technique termed modified resilient back propagation [16] is utilized for training both the action network and the critic network to approximate the 
optimal policy and the gradient of the cost to go function with respect to the states of the system in the online training phase. The modified resilient back propagation used for training preserves the information learned by the neural networks during the initialization phase.

\subsection{Thesis Organization}

The thesis is organized into six chapters. In this chapter the two phase design of the nonlinear neural controller is introduced. Chapter two introduces the concept of optimal control of nonlinear systems using neural networks. Chapter three introduces the concept of algebraic initialization of neural networks. Chapter four goes into the details of linear control design on which the nonlinear controller is based. Chapter four also compares the performance of the initialized neural controller with that of the linear controller operations with the nonlinear aircraft model. Chapter five gives an explanation of the algorithm used to implement adaptive critic designs. In chapter six, the neural networks are allowed to adapt online to account for nonlinearities which were ignored during the linear control designs. The response of the controller is evaluated using MATLAB simulations of a three degree of freedom general aviation aircraft in chapter six. The controller performance is evaluated with control failures and variations between the actual and assumed parameters. Chapter seven derives the conclusion of the thesis as well as suggests improvements and future work.

\subsection{Summary of results}

The nonlinear neural network controller is developed using the two stage process outlined

above. The nonlinear neural network controller is tested with the longitudinal nonlinear model of a general aviation aircraft. The results show that the controller improves performance in the presence of nonlinearities as compared to the linear controller design based on linearized model 
of the aircraft. The controller follows pilot inputs even in the presence of unanticipated control failures preserving stability for the closed loop system. Simulation results show that the controller preserves stability of the system and follows pilot input commands for large variations in $C_{m_{\alpha}}$ and $C_{D_{0}}$. 


\section{NEURAL CONTROL DESIGN}

The determination of an optimal control policy so as to minimize the cost of a metric is one of the fundamental problems of control theory. There exist closed form solutions for the determination of an optimal control policy for systems that are linear. Almost all systems of physical significance exhibit nonlinear behavior. Therefore there is a need for the design of a control system for a nonlinear plant so as to minimize a cost.

Since there exists much literature for the design of control systems for plants which are linear, nonlinear plants are linearized about different operating points to obtain a set of linear models covering the entire operating range. Linear controllers which then satisfy optimality criteria as well as robustness criteria can be designed for the each of the operating or design points. This method is followed widely in the industry and is termed gain scheduling. The controller gains obtained at different operating points for the linearized model are interpolated based on the operating conditions using a set of significant variables called scheduling variables.

Even the gain scheduled control systems do not always meet performance specifications when the operating conditions change rapidly. Furthermore if the number of scheduling variables increases it becomes extremely complex to switch or interpolate between the gains designed for the different operating points. Current research in the field of control theory focuses on the design of global controllers for nonlinear systems that can meet performance specifications in the presence of nonlinearities. Neural networks are parametric structures that can map nonlinear functions between multi dimensional inputs and outputs, and could be used to overcome the shortcomings of gain scheduled controllers.

There are different control architectures which use neural networks for control of nonlinear systems so as to meet performance specifications. An adaptive critic method is one of 
the architecture which could potentially be used to develop controllers which can meet performance specifications in the presence of nonlinearities and modeling errors. In this thesis following the work of Ferrari et al.[16], the aspects of linear control theory and neural networks for control are combined to develop an adaptive controller that is both robust and reconfigurable.

\subsection{Performance Measure}

Using classical control design, controllers can be designed for single input single output linear time invariant systems. The controller is evaluated based on some conditions like rise time, settling time, percentage overshoot, steady state error of the system in response to a step input. For multi input multi output systems it is necessary to evaluate controller performance objectives that cannot be readily described using classical terms. There is a need therefore to describe controller performance in a more general nature.

The optimal control problem involves determining the control policy $u^{*} \in U$, that causes the system represented in state form as $\dot{x}(t)=f(x(t), u(t), t)$ to minimize a metric or cost, which is a combination of the states and control signals,

$$
J=h\left(x\left(t_{f}\right), t_{f}\right)+\int_{t_{0}}^{t_{f}} g(x(t), u(t), t) d t
$$

Where $J$ is termed as the performance measure of the controller which gives the optimal control policy $u^{*}$. There are many ways in which the performance measure can be formulated. Some common performance measures are minimum time problems, terminal control problems, minimum control effort problems and Tracking problems. Based on which performance measure is used the optimal control policy minimizes a different cost or metric. 
For problems where the performance to be measured is to transfer a system from an initial state $x\left(t_{0}\right)=x_{0}$ to final state $x\left(t_{f}\right)=x_{f}$ in minimum time, the performance measure to be minimized by the controller is

$$
J=t_{f}-t_{0}
$$

For problems where we want to transfer the system from an initial state $x\left(t_{0}\right)=x_{0}$ to final state $x\left(t_{f}\right)=x_{f}$ by using least amount of control effort, the performance measure is given by

$$
J=\int_{t_{0}}^{t_{f}} u^{2}(t) d t
$$

where $u(t)$ is the control signal.

Similarly for problems where we need the states to follow a prescribed trajectory $r(t)$ in the time interval $\left[t_{0}, t_{f}\right]$ the performance measure is given by

$$
J=\int_{t_{0}}^{t_{f}}\|x(t)-r(t)\|^{2} d t
$$

The controller which minimizes the above cost is called an optimal controller. One other form of the cost function or performance measure is that we want to keep certain values of the states within prescribed limits. For example, we may want to control the longitudinal motion of an aircraft with the condition that the angle of attack of the aircraft should not go beyond the stall limit, the velocity, pitch rate and pitch angle should also remain within some maximum limit. This can be accomplished by incorporating these constraints into the performance measure as given below.

$$
J=\int_{t_{0}}^{t_{f}} \sqrt{\frac{V^{2}}{V^{2}{ }_{\text {max }}}+{\frac{q}{q^{2}{ }_{\text {max }}}}^{2}+{\frac{\theta}{\theta^{2}{ }_{\text {max }}}}^{2}+{\frac{\alpha}{\alpha^{2}{ }_{\text {max }}}}^{2}} d t
$$


When the controller is designed to minimize the above cost, it maintains the values of the state variables within prescribed limits. Thus we see that the performance measure can be used to specify the performance specifications the controller has to satisfy.

\subsection{Aircraft optimal control problem}

The dynamics of an aircraft are given by six nonlinear coupled ordinary differential equations, three for linear motion about the body fixed axes and the other three for rotations about the axes. The dynamics of the aircraft can be split into longitudinal motion and lateral directional motion. Longitudinal motion is a planar motion where the centre of mass of the aircraft is constrained to move in the vertical plane. When we consider only longitudinal motion of the aircraft we can uncouple the longitudinal equations of motion from the lateral equations of motion. In this thesis the adaptive controller is designed for longitudinal motion so we will henceforth deal with only three nonlinear ordinary differential equations of motion. In addition to the six nonlinear differential equations which are expressed in a coordinate system fixed to the center of mass of the aircraft, there are three auxiliary equations called the kinematic equations. The kinematic equations are used to determine the flight trajectory of the body fixed axis with respect to an inertial frame fixed to an arbitrary point on earth.

The model of the general aviation aircraft used in this thesis is obtained from actual physical and performance characteristics. The longitudinal motion of the aircraft is characterized by the nonlinearity of the drag model as well as nonlinearity in the lift produced due to high angle of attack $\alpha$. The aircraft can be linearized about different operating conditions based on the dynamically significant variables Altitude $(h)$ and velocity $(V)$. Initially a set of linear controllers are designed at linearized operating points, these are then replaced by neural networks whose performance is equivalent to the linear controllers they replace at all points of the operating 
envelope of the aircraft. This phase is called the pre-training phase of the aircraft. In the second phase the neural network parameters, i.e. the weights, are modified online to account for nonlinearities and modeling errors that were neglected during the design of the linear controller.

In the pre training phase we use LQR theory at each of the design points to determine the optimal controller to minimize the cost. Thus each LQR controller is designed for a selected operating point or equilibrium point in the flight envelope. The gain matrices associated with each LQR controller are used to define the architecture of the neural network as well as their initial weights. This process guarantees that the neural network controller performs exactly like the linear controller at each operating point.

In the online training phase the initialized neural networks are updated over time to closely approximate the globally optimal control law. The performance of the action neural network, denoted as $N N_{A}$, is evaluated by the critic neural network, denoted by $N N_{C}$. The adaptation is based on dual heuristic programming which also optimizes the same cost function which was optimized in the LQR or initialization phase.

\subsection{Linear Quadratic Regulator}

The nonlinear differential equation that models the longitudinal motion of the general aviation aircraft can be expressed as a function of the state vector $x(t)$, the parameters of the aircraft specified in terms of the stability derivatives, $p$, and the control inputs $u(t)$. The longitudinal states of the aircraft are the velocity $V$, the flight path angle $\gamma$, the pitch rate $q$ and the pitch angle $\theta$. The control inputs $u(t)$ to the aircraft are the thrust $T$ and the elevator deflection $\delta_{e}$. The state equation of the aircraft can be represented using the notation given below

$$
\dot{x}=f_{p}(x(t), u(t))
$$


The output equation for the longitudinal motion of the aircraft is given by

$$
y=g_{p}(x(t), u(t))
$$

In the thesis we make the assumption that there are ideal sensors available which accurately measure the state variables of the aircraft. The thrust and elevator deflection are the inputs to the aircraft longitudinal motion, thus the controller designed should provide the required thrust and elevator deflection to follow pilot commands for flight path angle and velocity i.e. $y_{c}(t)=\left[\begin{array}{ll}V & \gamma\end{array}\right]$. The control law for $u(t)$ is a function of the current state $x(t)$

$$
u(t)=c(x(t))
$$

The control law $c$ may contain functions of its arguments such as integrals and derivatives. Furthermore the control signal may be written as a sum of the nominal steady state value and perturbed effects, i.e. $u(t)$ can be written as

$$
\begin{aligned}
& u(t)=c_{0}\left(x_{0}\right)+\Delta c(\Delta x(t)) \\
& u(t)=u_{0}+\Delta u(t)
\end{aligned}
$$

where $x_{0}$ is the nominal value of the state and $\Delta x(t)$ is the perturbed value, i.e. $x(t)=x_{0}+\Delta x(t)$. The control law is then given by

$$
u(t)=c_{0}\left(x_{0}\right)+\Delta c\left(x_{0}, \Delta x(t)\right)
$$

For small perturbations in the state, the control perturbation may be approximated by a linear relationship

$$
\Delta u(t)=-C \Delta x
$$

Where $C$ is the gain matrix associated with the control law at $x_{0}$.

In the pre training phase the goal is to determine the architecture and the initial weights of the action neural network, $N N_{A}$, based on linear control design. This network will gain schedule 
the control over the entire envelope. In gain scheduling the nonlinear equations of motion of the aircraft are linearized about equilibrium points called design points. At each equilibrium point the following condition holds

$$
0=f_{p}\left(x_{0}(a) u_{0}(a)\right)=\dot{x}
$$

where $a$ is the scheduling vector with the dynamically significant variables velocity and altitude. A set of equilibrium points for which the above condition holds can be obtained for the nonlinear aircraft model. The linearized model of the aircraft at each of the $K$ operating points $(O P)$ can be obtained by assuming small perturbation about each of the operating points. This results in a set of $K$ linear models of the form

$$
\Delta \dot{x}(t)=F \Delta x(t)+G \Delta u(t)
$$

Now a set of controllers are to be designed for the linear models at each operating point considered in the flight envelope such that the performance measure of the form

$$
J=\Phi\left(x, t_{f}\right)+\frac{1}{2} \int_{0}^{t_{f}}\left[\Delta x^{T}(\tau) Q \Delta x(\tau)+2 \Delta x^{T} M \Delta u(\tau)+\Delta u^{T}(\tau) R \Delta u(\tau)\right] d \tau
$$

is minimized. The matrices $Q, M$ and $R$ are called the weighting matrices and are selected by the designer. At any moment in time, $t_{0}<t<t_{f}$, a "cost-to-go" function or "value function" can be defined and is given by

$$
V[\Delta x, t]=\Phi\left(x, t_{f}\right)+\frac{1}{2} \int_{t_{0}}^{t}\left[\Delta x^{T}(\tau) Q \Delta x(\tau)+2 \Delta x^{T} M \Delta u(\tau)+\Delta u^{T}(\tau) R \Delta u(\tau)\right] d \tau
$$

The relationship between the cost function and the value function is given in Figure 5. In the infinite horizon case considered in this thesis, it can be assumed that the terminal cost $\Phi\left(x, t_{f}\right)$ is zero [18]. 

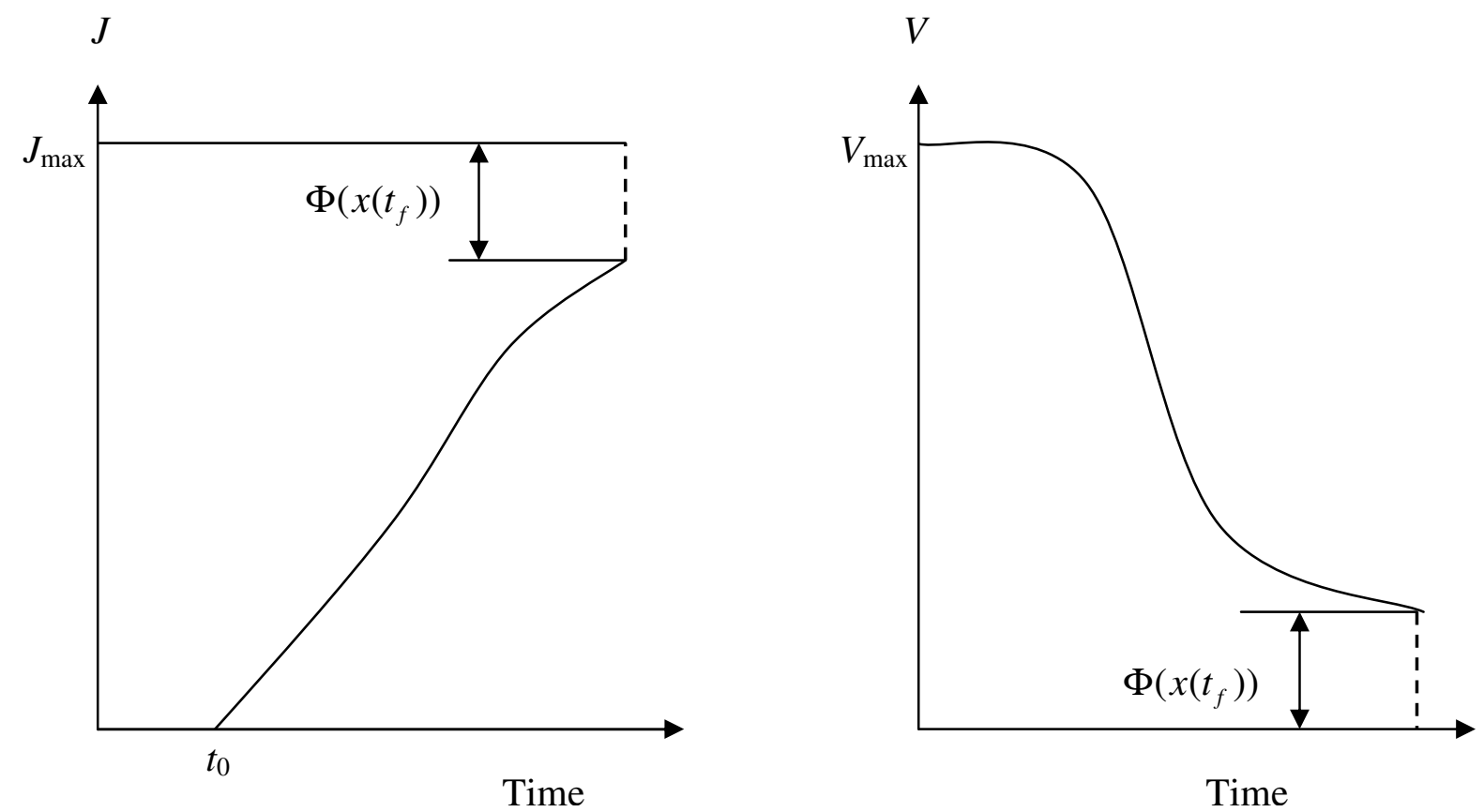

Figure 5: Relationship between Cost function and Value function

The term inside the integral in the above equations is termed the Lagrangian and is denoted by

$$
L(\Delta x, \Delta u, t)=\frac{1}{2}\left[\Delta x^{T}(t) Q \Delta x(t)+2 \Delta x^{T}(t) M \Delta u(t)+\Delta u^{T}(t) R \Delta u(t)\right]
$$

Now the value function can be rewritten using the definition of the Lagrangian as

$$
V(\Delta x, t)=\int_{t_{0}}^{t} L[\Delta x, \Delta u, \tau] d \tau
$$


The minimum value function from the current time $t$ to the final time $t_{f}$ is obtained when the control signal $\Delta u(t)$ is equal to the optimal control signal $\Delta u^{*}(t)$. When the control is optimal, the resulting states from the time $t$ to $t_{f}$ lie on the optimal state trajectory denoted by $\Delta x^{*}(t)$ i.e.

$$
V^{*}\left(\Delta x^{*}, t\right)=\int_{t_{0}}^{t} L\left[\Delta x^{*}, \Delta u^{*}, \tau\right] d \tau
$$

Where $(\bullet)^{*}$ denotes the optimal value. The critic network in the adaptive critic design approximates the gradient of this value function or cost to go function with respect to the states of the system at each instant of time. This serves as an evaluation of the action network which when trained approximates the optimal control law $\Delta u^{*}(t)$ which minimizes the cost function.

For systems whose dynamics are linear and the performance measure is quadratic an optimal closed form solution can be obtained for the control perturbation $\Delta u$. One way of obtaining the closed form solution is based on the principles of calculation of variations.

The optimization problem given above is a constrained minimization problem, i.e. the minimization of the performance measure is subject to constraints on the state and control. This problem is converted to an unconstrained minimization problem using the concept of Lagrange multipliers resulting in the Hamiltonian of the system

$$
H(\Delta x, \Delta u, \lambda, t) \equiv L(\Delta x, \Delta u, t)+\lambda^{T}(t) f_{p}(\Delta x, \Delta u, t)
$$

When the control signal $u(t)$ is equal to the optimal value $u^{*}(t)$, the resulting states as well as the lagrange multiplier are also optimal, i.e. $\Delta x(t)=\Delta x^{*}(t)$ and $\lambda^{T}(t)=\lambda^{*^{T}}(t)$. Therefore the Hamiltonian is written as

$$
H\left(\Delta x^{*}, \Delta u^{*}, \lambda^{*}, t\right) \equiv L\left(\Delta x^{*}, \Delta u^{*}, t\right)+\lambda^{* T}(t) f_{p}\left(\Delta x^{*}, \Delta u^{*}, t\right)
$$


On differentiating the optimal cost to go function defined in equation (20) with respect to time we have

$$
\frac{d V^{*}\left(\Delta x^{*}, t\right)}{d t}=\frac{\partial V^{*}\left(\Delta x^{*}, t\right)}{\partial t}+\frac{\partial V^{*}\left(\Delta x^{*}, t\right)}{\partial \Delta x} \frac{d \Delta x}{d t}
$$

also we have

$$
\frac{d V^{*}}{d t}=L\left[\Delta x^{*}, \Delta u^{*}, t\right]
$$

therefore from equation (23), (24) and (14), the partial derivative of the optimal cost to go function w.r.t to $t$ can be written as

$$
\frac{\partial V^{*}}{\partial t}\left(\Delta x^{*}, t\right)=L\left(\Delta x^{*}, \Delta u^{*}, t\right)+\frac{\partial V^{*}}{\partial \Delta x}\left(\Delta x^{*}, t\right) f_{p}\left(\Delta x^{*}, \Delta u^{*}, t\right)
$$

On comparing equation (22) and (25) we can now relate the partial derivative of the value function with respect to time and the Hamiltonian resulting in the Hamilton-Jacobi-Bellman equation

$$
\frac{d V^{*}}{d t}=H\left\{\Delta x^{*}, \Delta u^{*}, \frac{\partial V^{*}}{\partial \Delta x}\left(\Delta x^{*}, t\right), t\right\}
$$

On comparing equation (26) with the optimal Hamiltonian of the system we see that the optimal adjoint vector $\lambda^{* T}$ is equal to the gradient of the cost to go function with respect to the state. The critic network is set up to approximate the optimal adjoint vector when trained to output the gradient of the cost to go function, i.e.

$$
\lambda^{*}(t)=\frac{\partial V^{*}\left(\Delta x^{*}, t\right)}{\partial \Delta x^{*}}
$$

For linear systems with quadratic cost functions, $\Delta u^{*}(t)$ can be found as a function of $\Delta x$ and equation (17) can be written as the optimal value function given by [18] 


$$
V^{*}\left(\Delta x^{*}(t)\right)=\frac{1}{2} \Delta x^{* T}(t) P(t) \Delta x^{*}(t)
$$

The matrix $P(t)$ is positive definite and for linear time invariant systems reaches steady state as $t$ approaches $\infty$. To obtain the optimal control perturbation to minimize the cost, the Hamiltonian is differentiated with respect to $\Delta u$ and set equal to zero. Now the optimal value or cost function is differentiated with respect to $\Delta x^{*}$ and $t$. The Hamilton-Jacobi-Bellman equation reduces to

$$
\Delta x^{*^{T}}(t) M+\Delta u(t)^{*^{T}} R+\Delta x^{* T}(t) P(t) G=0
$$

which can now be solved for the optimal control perturbation $\Delta u(t)^{*}$

$$
\Delta u^{*}(t)=-R^{-1}\left(G^{T} P+M^{T}\right) \Delta x^{*}(t)=-C \Delta x^{*}(t)
$$

The Riccati equation given by equation (31) is then used find $P$ [18].

$$
\dot{P}(t)=-\left(F-G R^{-1} M^{T}\right) P(t)-P(t)\left(F-G R^{-1} M^{T}\right)+P(t) G R^{-1} G^{T} P(t)-Q+M R^{-1} M^{T}
$$

Now linear time invariant controllers are designed for a set of models $\{F, G\}_{k=1 \ldots . . K}$ developed about each of the equilibrium or operating points. This leads to a set $K$ of gain and Riccati matrices $\{C, P\}_{k=1 \ldots \ldots . K}$ which are indexed based on the operating point. In conventional gain scheduling the gain matrices are stored in a computer with different gains being selected based on the scheduling variables. For operating conditions which are in between two operating points, the gains are decided by interpolation.

For the nonlinear neural network based controller, the set of above gains are used to initialize a neural network. By using the gains developed for the linear model at different operating points, it is possible for us to initialize the neural network such that they meet the performance specifications met by the linear controllers at the operating points considered. Further since neural networks can generalize the multi dimensional input output relationship, 
their performance for points in between the operating points considered exceeds that of the gain scheduled design.

\subsection{Nonlinear neural controller}

The nonlinear neural network controller is based on the adaptive critic architecture. The adaptive critic architecture consists of two neural networks called the action, $N N_{A}$ and the critic, $N N_{C}$. The action neural network approximates the optimal control function whereas the critic neural network measures the performance of the action neural network. A key feature of the neural networks that replace the gain matrices is that the gradients of the function represented by the neural networks must equal corresponding optimal gains that were derived form the linear quadratic law developed in the previous section. This can be seen from equation (13). The nonlinear neural network controller architecture is as shown in Figure 6.

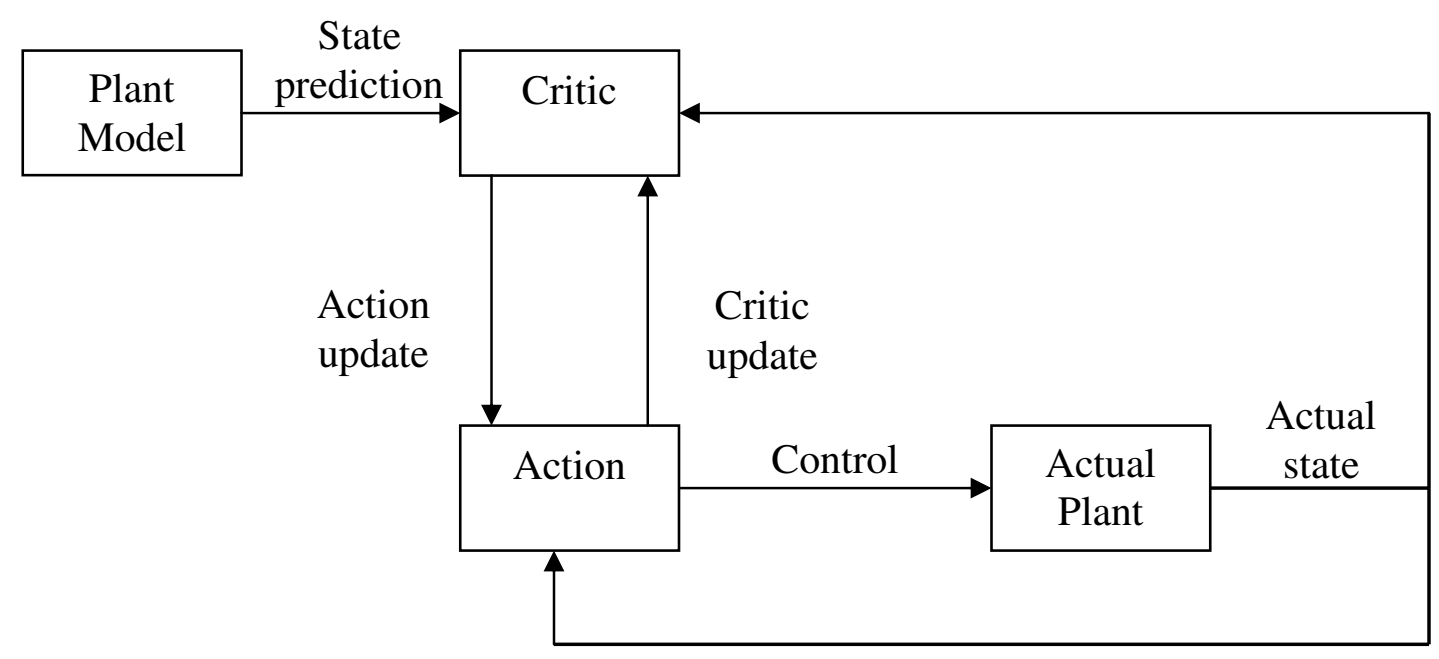

Figure 6: Adaptive critic based neural network controller. 
The output of a neural network is a nonlinear function of the input and is based on the architecture and the weights of the neural network. Let $z$ represent the output of a neural network, and then the output $z$ is given by the nonlinear function denoted by $N N$, of its input vector $p$, i.e.

$$
z=N N(p)
$$

Now the output of the action network in the controller architecture is the approximation of the optimal control signal $u^{*}(t)$ denoted by $u(t)$. The critic network approximates, $\lambda^{*}(t)$ denoted by $\lambda(t)$, the gradient of the optimal cost go function with respect to the state. Then using the above representation for the neural network outputs we can write

$$
\begin{aligned}
& u(t)=N N_{A}\left(\left[x(t)^{T}, a^{T}\right]^{T}\right)=z_{A}(t) \\
& \lambda(t)=N N_{C}\left(\left[x(t)^{T}, a^{T}\right]^{T}\right)=z_{C}(t)
\end{aligned}
$$

where $x$ is the state of the system and $a$ is the dynamically significant scheduling vector consisting of the velocity $V$ and the altitude $h$. In the pre training phase we need to incorporate the gains obtained using the LQR law into the nonlinear neural networks.

For each of the $K$ operating points considered, there is a corresponding gain matrix $C^{k}$ and a Riccati matrix $P^{k}$. At each of the $K$ operating points, the gradient of the action network is given by

$$
\left.\frac{\partial z_{A}(t)}{\partial x(t)}\right|_{x=x_{0}^{k}, a=a^{k}}=\left.\frac{\partial u(t)}{\partial x(t)}\right|_{x=x_{0}^{k}, a=a^{k}}=\left.\frac{\partial \Delta u^{*}(t)}{\partial \Delta x^{*}(t)}\right|_{x=x_{0}^{k}, a=a^{k}}
$$

which is equal to the corresponding gain matrix $C^{k}$.

$$
\left.\frac{\partial z_{A}(t)}{\partial x(t)}\right|_{x=x_{0}^{k}, a=a^{k}}=-\left.C^{k}\right|_{x=x_{0}^{k}, a=a^{k}}
$$


This is true for every operating point considered in the design of the linear quadratic controller, Now the total control is obtained by adding $u_{0}^{k}$, which is the trim value, to the output of the action network.

The critic must approximate the optimal adjoint vector $\lambda^{*}(t)$. The optimal value function of equation (28) can be differentiated twice with respect to the state at each of the operating points considered to obtain the following.

$$
\left.\frac{\partial z_{c}(t)}{\partial x(t)}\right|_{x=x_{0}^{k}, a=a^{k}}=\left.\frac{\partial \lambda(t)}{\partial x(t)}\right|_{x=x_{0}^{k}, a=a^{k}}=\left.\frac{\partial^{2} V^{*}\left(\Delta x^{*}, t\right)}{\partial \Delta x(t)^{2}}\right|_{x=x_{0}^{k}, a=a^{k}}
$$

This is equivalent to the steady state Riccati matrix $P^{k}$ for each and every operating point considered in the design of the linear quadratic controller.

$$
\left.\frac{\partial z_{C}(t)}{\partial x(t)}\right|_{x=x_{0}^{k}, a=a^{k}}=P^{k}
$$

In addition at the operating points considered in the design of the linear quadratic controller the outputs of the neural networks must be equal to zero, i.e.

$$
\left.z(x(t), a)\right|_{x=x_{0}^{k}, a=a^{k}}=0
$$

when these conditions on the value and the gradient, equations (36) and (39) of each $N N$ are satisfied, the neural network can be initialized as explained in chapter three to perform exactly equal to the linear quadratic controller designed for each and every operating point.

\subsection{Adaptive critic fundamentals}

The adaptive critic architecture is based on dual heuristic programming. In this the pretrained neural networks are updated online over time to approximate the globally optimal control law. The adaptation improves control response for nonlinearities as well as control failures and 
modeling errors. The updating of the action and critic neural network are implemented in discrete time through an incremental optimization scheme called dual heuristic programming. Dual heuristic programming is based on the recurrence relation of dynamic programming. The nonlinear continuous system represented by

$$
\dot{x}=f_{p}(x, u, t)
$$

is discretized assuming piecewise constant inputs and constant time interval $\Delta t$. The discrete equivalent of the plant is then given by

$$
x\left(t_{k}+1\right)=f_{p D}\left(x, u, t_{k}\right)
$$

In the online phase the same metric that is optimized in the offline training phase is minimized providing a systematic approach to control design. As with the plant the cost function also needs to be discretized. The discrete equivalent of the cost of equation (16) is given by

$$
J_{0, N}=\sum_{k=0}^{N-1} L_{D}\left[x, u, t_{k}\right] \Delta t, N \Delta t \rightarrow \infty
$$

where the discrete form of the Lagrangian in equation (19) is made use of. The integral in the time domain is converted to the summation in the discrete domain. As in the continuous time domain, a value function can also be defined for the discrete time domain. The cost of going from the $k^{\text {th }}$ instant, $t_{k}$, to the final instant of time $t_{N}$ can be written as

$$
V\left(x, t_{k}\right)=\left(L_{D}\left[x, u, t_{k}\right]+L_{D}\left[x, u, t_{k+1}\right]+\ldots \ldots \ldots+L_{D}\left[x, u, t_{N-1}\right]\right) \Delta t
$$

But from the discrete state equation, the state $x\left(t_{k+1}\right)$ depends on the current state $x\left(t_{k}\right)$ and the current control signal $u\left(t_{k}\right)$, therefore the cost to go function can be written as

$$
V\left(x, t_{k}\right)=L_{D}\left[x, u, t_{k}\right] \Delta t+V\left(x, t_{k+1}\right)\left[x\left(t_{k+1}\right), u\left(t_{k+1}\right), \ldots \ldots . u\left(t_{N-1}\right)\right]
$$


The objective is now to find a sequence of control such that the cost function or performance measure is minimized. Therefore the optimal cost for the $(N-k)$ stage policy is found by minimizing the following functional with respect to the control sequence

$$
V^{*}\left[x^{*}, t_{k}\right] \equiv \min _{u\left(t_{k}\right) \ldots \ldots . u\left(t_{N-1}\right)}\left\{V\left[x^{*}\left(t_{k}\right), u\left(t_{k}\right), \ldots \ldots \ldots u\left(t_{N-1}\right)\right\}\right.
$$

From the principle of optimality if a policy is optimal over $(N-k)$ stages what ever the initial state and decision are the remaining decisions must also constitute an optimal policy for the state obtained from the first decision, i.e. $x\left(t_{k+1}\right)$, then from equations (44) and (45)

$$
V^{*}\left[x, t_{k}\right] \equiv \min _{u\left(t_{k}\right)}\left\{L_{D}\left[x, u, t_{k}\right] \Delta t+V^{*}\left[x^{*}, t_{k+1}\right]\right\}
$$

This equation is called the recurrence relation of dynamic programming. In normal dynamic programming the above equation is solved backwards in time starting from the final time to obtain the optimal control sequence. In forward dynamic programming we use Howard's form of recurrence relation given by

$$
V\left[x, t_{k}\right]=L_{D}\left[x, u, t_{k}\right] \Delta t+V\left[x, t_{k+1}\right]
$$

where the cost $V\left[x, t_{k+1}\right]$ to go from the state $x\left(t_{k+1}\right)$ is a predicted or estimated value obtained from the critic network. The approximate optimal control at any time, $t_{k}, u\left(t_{k}\right)$ is defined as the one that minimizes the equation (47) for any $x\left(t_{k}\right)$. It has been shown that when the control $u\left(t_{k}\right)$ is calculated to minimize the cost to go function $V\left[x, t_{k}\right]$ based on the above equation the method converges to the optimal solution over time [19] if the state space is explored completely and infinitely often.

The control $u\left(t_{k}\right)$ that for which the value function is stationary is the optimal control strategy that minimizes the value function, i.e. 


$$
\frac{\partial V\left[x, t_{k}\right]}{\partial u\left(t_{k}\right)}=\frac{\partial L_{D}\left[x, u, t_{k}\right]}{\partial u\left(t_{k}\right)}+\lambda\left(t_{k+1}\right) \frac{\partial x\left(t_{k+1}\right)}{\partial u\left(t_{k}\right)}=0
$$

Recall $\lambda=\frac{\partial V}{\partial x}$ is the output of the critic network. Since the action network has to generate the control signal to minimize the cost, equation (48) is used to find $u\left(t_{k}\right)$ that is the target for the adaptation of the action neural network. Note that the critic network is necessary to give $\lambda\left(t_{k+1}\right)$ to solve equation (48). In addition a model of the plant is necessary to evaluate the transition matrix $\frac{\partial x\left(t_{k+1}\right)}{\partial u\left(t_{k}\right)}$. The solution of equation (48) gives the optimal control $u\left(t_{k}\right)$. To obtain the recurrence relation for finding the target value for the critic network training Howard's form of the optimal value function is differentiated with respect to the state to obtain

$$
\lambda\left(t_{k}\right) \equiv \frac{\partial V\left[x, t_{k}\right]}{\partial x\left(t_{k}\right)}=\frac{\partial L_{D}\left[x, u, t_{k}\right]}{\partial x\left(t_{k}\right)}+\frac{\partial L_{D}\left[x, u, t_{k}\right]}{\partial u\left(t_{k}\right)} \frac{\partial u\left[x\left(t_{k}\right)\right]}{\partial x\left(t_{k}\right)}+\lambda\left(t_{k+1}\right) \frac{\partial x\left(t_{k+1}\right)}{\partial x\left(t_{k}\right)}+\lambda\left(t_{k+1}\right) \frac{\partial x\left(t_{k+1}\right)}{\partial u\left(t_{k}\right)} \frac{\partial u\left[x\left(t_{k}\right)\right]}{\partial x\left(t_{k}\right)}
$$

This constitutes the adaptation criteria for the critic network. Equation (49) also requires a model of the plant in order to determine the transition matrices $\frac{\partial x\left(t_{k+1}\right)}{\partial x\left(t_{k}\right)}$ and $\frac{\partial x\left(t_{k+1}\right)}{\partial u\left(t_{k}\right)}$. When the adaptation for both the critic and action network are carried out iteratively over time the action network converges to the optimal control policy. 


\section{ALGEBRAIC TRAINING OF NEURAL NETWORKS}

Neural networks are parallel computational paradigms that can approximate nonlinear mappings between multi dimensional inputs and outputs with relatively small error. Neural networks are based loosely on biological neural formations and are characterized by their architecture, the number of neurons and in the order in which they are arranged, and the weights between their interconnections.

The architecture of the neural network to approximate a given nonlinear mapping between the input and output is not unique. It is possible to achieve arbitrarily low error between the desired output and actual output of a network using different kinds of network architecture. In this chapter, a method is described to determine the architecture and the initial weights of a neural network by solving a set of linear equations to approximate a nonlinear mapping between the input and output. This method was developed by Ferrari [16, 20, and 21]. A particular case that is relevant to control system design used in this thesis is explained in detail.

A set of nonlinear equations to be solved for the neural network adjustable parameters is obtained by using the training set of inputs and outputs and the gradient of the output with respect to either all or some of the inputs. The function to be approximated is usually not known analytically, but the set of inputs, $y^{k}$, corresponding outputs, $u^{k}$ and gradients $C^{k}$ are available in the form $\left\{y^{k}, u^{k}, C^{k}\right\}_{k=1, \ldots . K}$. The neural network approximates the functional relationship $u^{k}=g\left(y^{k}\right)$ between the input and output. The neural network can improve generalization if the gradient information $C^{k}$ is also used [16]; hence they are also incorporated into the training set.

The output of a neural network of the form shown in Figure 7 is given by the nonlinear equation 


$$
z=v^{T} \sigma(W p+d)+b
$$

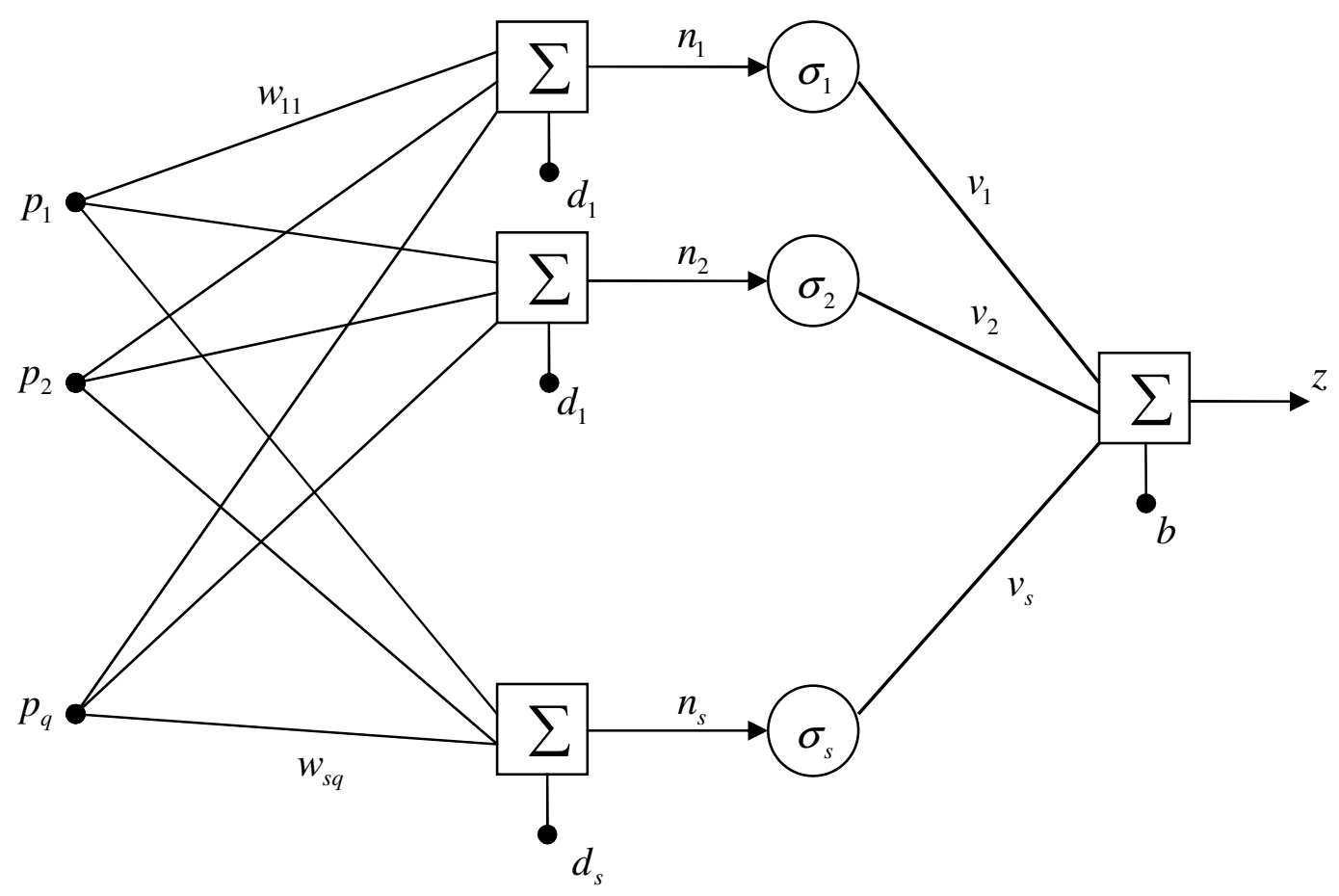

Figure 7: Sigmoidal neural network.

Where $p$ is the input vector and $z$ is the output of the neural network. The activation function used in the neural network is the sigmoidal function given by $\sigma(x)=\frac{e^{x}-1}{e^{x}+1}$ where $\mathrm{x}$ is the input to the sigmoidal function. In the neural network shown in Figure 7, the input to the sigmoidal function is $(W p+d)$ which is represented as $n$, i.e.

$$
n \equiv(W p+d)
$$

The nonlinear nature of the neural network is due the nonlinearity of the activation function used in the neurons. 
The input weights $W$, the output weights $v$, together with the input bias $d$ and $b$ constitute the parameters of the neural network that can be adjusted to approximate the input output relationship. To match a given input output set the neural network output must satisfy the nonlinear equation

$$
u^{k}=v^{T} \sigma\left(W y^{k}+d\right)+b, k=1,2, \ldots . K
$$

which can be represented by a vector equation for all the $K$ training pairs.

$$
u=S v+b
$$

$\boldsymbol{b}$ is an s-dimensional vector, equal to the number of neurons in the neural network, composed of the scalar output bias, $b . S$ is a matrix of sigmoidal functions evaluated at input to node values, $n_{i}^{k}$, each representing the magnitude of the input-to-node variable.

$$
S \equiv\left[\begin{array}{ccc}
\sigma\left(n_{1}^{1}\right) & \sigma\left(n_{2}^{1}\right) \cdots & \sigma\left(n_{s}^{1}\right) \\
\sigma\left(n_{1}^{2}\right) & \sigma\left(n_{2}^{2}\right) \cdots & \sigma\left(n_{s}^{2}\right) \\
\vdots & \vdots & \vdots \\
\sigma\left(n_{1}^{K}\right) & \sigma\left(n_{1}^{K}\right) \cdots & \sigma\left(n_{1}^{K}\right)
\end{array}\right]
$$

The known gradient, $c^{k}$, corresponds to the partial derivatives of the neural network's output with respect to its inputs evaluated at the training pair $k$. Exact matching of the gradient set is obtained if the neural network satisfies the gradient equation[16].

$$
c^{k}=W(\bullet, 1 \rightarrow e)^{T}\left\{v \otimes \sigma^{\prime}\left(n^{k}\right)\right\}, k=1, \ldots . K
$$

Where the symbol " $\circledast$ " denotes element-wise vector multiplication, and $W(\bullet, 1 \rightarrow e)$ represents the first $e$ columns of the input weight matrix for which the gradients are known. Equation (54) can be rewritten as

$$
c^{k}=\left[B^{k} W(\bullet, 1 \rightarrow e)\right]^{T}, k=1, \ldots . K
$$

With the matrix 


$$
B^{k} \equiv\left[v_{1} \sigma^{\prime}\left(n_{1}^{k}\right) \quad v_{2} \sigma^{\prime}\left(n_{2}^{k}\right) \quad \ldots \ldots . \quad v_{s} \sigma^{\prime}\left(n_{s}^{k}\right)\right], k=1, \ldots . K
$$

\subsection{Exact gradient based solution}

As stated earlier for the neural network based nonlinear controller used in this thesis to perform as well as the gain matrices they replace, the gradient of the function defined by the neural network should equal the gains at the corresponding operating points. At the operating point the output of the neural networks is zero since the perturbed input states $x(t)$ is zero. Therefore the training pairs are a set of points of the form $\left\{y^{k}, 0, c^{k}\right\}_{k=1,2 . . K}$. The input to the network can be partitioned at the design points into $y^{k}=\left.\left[x^{k}=0 \mid a^{k^{T}}\right]^{T}\right|_{k=1,2 \ldots K}$ as both the states of the system and scheduling variables are fed as inputs to the neural network. Therefore the training set can be written as $\left\{\left[0 \mid a^{k^{T}}\right]^{T}, 0, c^{k}\right\}_{k=1,2 \ldots K}$. Now the output of the neural network can be written using the nonlinear equation (50) as

$$
0=v^{T} \sigma\left(\left[W_{x} \mid W_{a}\right]\left[0 \mid a^{k^{T}}\right]^{T}+d\right)+b, k=1,2 \ldots K
$$

Now we see that the output equation does not depend on the weights associated with the perturbed states $x(t)$, and the equation reduces to

$$
0=v^{T} \sigma\left(\left[W_{a}\right]\left[a^{k^{T}}\right]^{T}+d\right)+b, k=1,2 \ldots K
$$

Now the gradient equation (54) can be written as

$$
c^{k}=W_{x}^{T}\left\{v \otimes \sigma^{\prime}\left(\left[W_{a}\right]\left[a^{k^{T}}\right]^{T}+d\right\}, k=1,2 \ldots K\right.
$$

Since the gradient of the function is known only for the state and not for the scheduling variables only the weights $W_{x}$, associated with the states are considered. From equation (51), the input to node values $n$ can be represented as 


$$
n_{i}^{k} \equiv W_{a} a^{k}+d, k=1,2 \ldots K, i=1,2 \ldots \ldots s
$$

Now if we know all the inputs to the hidden nodes the output equation becomes linear in the output weights and is given by

$$
b=-S v
$$

If we choose the number of nodes in the hidden layer equal to the number of training pairs, the matrix $S$ is square, the above equation can then be solved for a unique $v$ for an arbitrary $b$ if $S$ is also non singular. In the thesis the vector $b$ is generated using MATLAB function rand.

Now the gradient equation (59) can also be treated as linear and be represented as

$$
C=X w_{x}
$$

The unknowns in the above equation consist of the input weights $w_{x}$. The matrix $\mathrm{w}_{x}$ consists of the weight matrix $W_{x}$ rearranged into a vector form, which consists of column wise reorganization of the matrix elements into a vector [16]. The vector $C$ is the known gradients in the training set,

$$
C \equiv\left[c^{1^{T}}|\ldots .| c^{K^{T}}\right]^{T}
$$

and $X$ denotes a sparse matrix composed of block diagonal submatrices each of size $e \times e s$

$$
X \equiv\left[\begin{array}{ccccc}
B^{1} & 0 & 0 & 0 & 0 \\
0 & B^{1} & 0 & 0 & 0 \\
: & : & : & \vdots & : \\
0 & 0 & 0 & 0 & B^{1} \\
- & - & - & - & - \\
\vdots & \vdots & \vdots & \vdots & \vdots \\
- & - & - & - & - \\
B^{p} & 0 & 0 & 0 & 0 \\
0 & B^{p} & 0 & 0 & 0 \\
: & \vdots & : & \vdots & : \\
0 & 0 & 0 & 0 & B^{p}
\end{array}\right]
$$


Every block $B^{k}$ is known when $v$ and all input-to-node values are known from equation (56) and $n^{k}$ depends only on $W_{a}$, furthermore when the number of hidden nodes is equal to the number of training pairs, $X$ is a square matrix and can be solved to give a unique value for $w_{x}$ provided $X$ is also non singular.

The third set of linear equations is obtained from the assumption that the input to node values is known for all the nodes and at all training pairs. The input to node values $n_{i}^{k}$ are rearranged as an array as follows

$$
\eta=\left[n^{1^{T}}|\ldots . .| n^{K^{T}}\right]^{T}
$$

The linear equation $n_{i}^{k}=W_{a} a^{k}+d$ can be written as

$$
\eta=A w_{a}
$$

Where the matrix $A$ is given by

$$
A \equiv\left[\begin{array}{cccc}
a_{1}^{1} I_{s} & \cdots & a_{(q-e)}^{1} I_{s} & I_{s} \\
a_{1}^{2} I_{s} & \cdots & a_{(q-e)}^{1} I_{s} & I_{s} \\
\vdots & \cdots & \vdots & \vdots \\
a_{1}{ }^{K} I_{s} & \cdots & a_{(q-e)}^{1} I_{s} & I_{s}
\end{array}\right]
$$

The matrix $w_{a}$ is obtained by rearranging the weights $W_{a}$ and the input bias vector $d$ as $w_{a}=\operatorname{Vec}\left[W_{a} \mid d\right]$. When the number of training sets is equal to the number of hidden nodes, $\eta$ is a known vector which is related to the input weights associated with the scheduling variable, $W_{a}$ and the input bias, $d$.

These linear equations are solved such that the neural network parameters, $W, v, d, b$ are obtained to match the training set exactly. The first equation to be solved is the input to node value equations, followed by the output weight equations and the gradient weight equations. 
Since the number of nodes, $s$, is equal to the number of training pairs, $p, A$ and $C$ are determined from the equations (67) and (66) respectively. The vector $\eta$ is determined so that the matrix $S$ is well conditioned. One strategy for producing a well conditioned $S$ is to generate the vector $\eta$ as follows

$$
n_{i}^{k}=\left\{\begin{array}{lr}
r_{i}^{k}, i \neq k \\
0 \quad i=k
\end{array}\right.
$$

where $r_{i}^{k}$ is chosen from a normal distribution with mean zero and variance one. Now an estimate of $w_{a}$ can be obtained by solving the following equation

$$
\hat{w}_{a}=A^{P I} \eta
$$

$\hat{w}_{a}$ is the best approximation to the solution. When this value for $w_{a}$ is substituted into the equation

$$
\hat{\eta}=A \hat{w}_{a}
$$

we get the vector $\hat{\eta}$ and from this $\sigma[n] \equiv\left[\sigma\left(n_{1}\right) \ldots \ldots . . \sigma\left(n_{s}\right)\right]^{T}$ is obtained. Now $S$ can be computed from the vector $\eta=f \hat{\eta}$, where $f$ is a factor such that each sigmoid is centered for a given input $y^{k}$ and is close to saturation for any other known input. The weight matrix $w_{a}$ is now obtained using the relation

$$
w_{a}=f \hat{w}_{a}
$$

Once $S$ is known the output weight matrix $v$ can be obtained. With the solution of $v$ and $\eta$, the matrix $X$ can be formed from which the weight $w_{x}$ can be obtained.

Thus the neural network can be initialized guaranteeing that the neural networks meet the performance specification of the linear gain matrices that they replace. This concludes the initialization phase of the adaptive critic design. 


\section{LINEAR CONTROL DESIGN}

The neural network controller is based on a linear controller which is designed using linear control theory for a set of models linearized about equilibrium points. In this chapter the design process of a linear controller for the longitudinal model of an aircraft is explained. The linear controller establishes the performance requirements to be met by the system. As explained

in chapter three the linear controllers are used to determine the architecture and initial weights of the neural network. When the neural networks are initialized as outlined in chapter three the neural network controller performs as well as the linear controllers that they replace at the design points .

Simulations carried out with the longitudinal model show that the initialized neural network controller performs as well as the linear controller designed at the operating points. The neural network based controller is also able to perform as well as a gain scheduled linear controller at interpolation points between the design points considered in the operating envelope of the aircraft. The online training phase improves the controller's performance in the presence of nonlinearities as well as for unanticipated control failures and parameter variations between the actual model and the assumed model. The online training phase of the controller is described in chapter five.

The operating envelope of the aircraft is as shown in Figure 8. It can be divided into the set of operating points, called $O P$ considered in the initialization phase. The points that are within the area enclosed by the design points considered in the initialization phase is called the interpolating region abbreviated as $I R$. The region which is outside the area enclosed by the design points considered in the initialization phase is called the extrapolating region $E R$. 


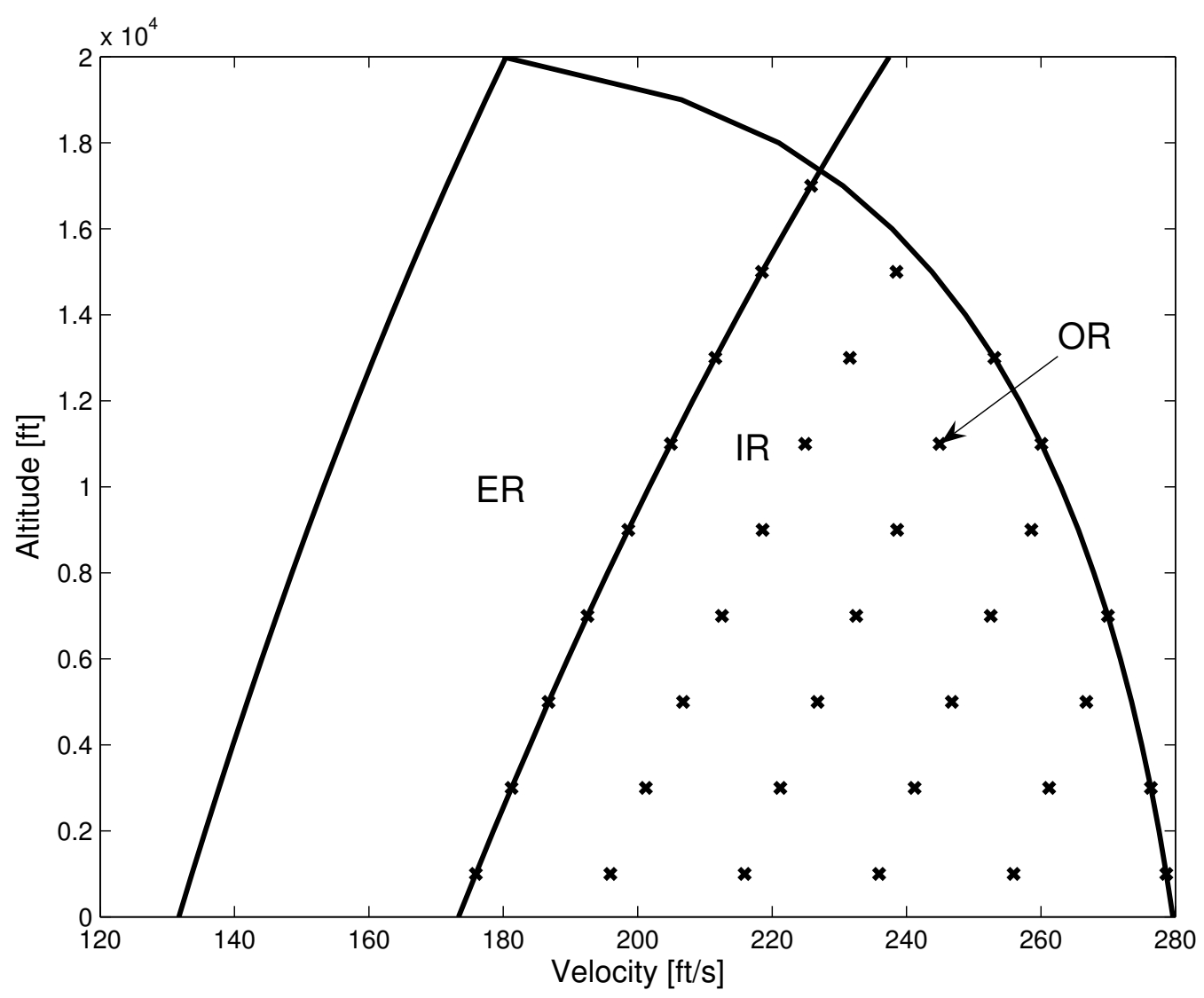

Figure 8: Operating envelope

The longitudinal model of the aircraft is available in terms of the stability derivatives. Since only the longitudinal model of the aircraft is available the controller is designed to follow longitudinal pilot commands for the flight path angle $\gamma$ and forward velocity $V$. The controller is designed using the state vector $x=\left[\begin{array}{lllll}V & \gamma & q & \theta\end{array}\right]^{T}$ comprising the airspeed, flight path angle, the pitch rate and the pitch angle. The scheduling vector $a=\left[\begin{array}{ll}V & h\end{array}\right]$ is comprised of the airspeed and altitude respectively. The control inputs to the aircraft to control longitudinal flight are the throttle and the elevator, therefore the control vector $u$ is given by $u=\left[\begin{array}{ll}\delta_{T} & \delta_{e}\end{array}\right]^{T}$. The pilot command is represented by the vector $y_{C}=\left[\begin{array}{ll}V_{C} & \gamma_{C}\end{array}\right]^{T}$. 


\subsection{Flight envelope}

The operating envelope of the general aviation aircraft needs to be determined based on the stall speed of the aircraft, the thrust available and the maximum allowable dynamic pressure to prevent structural damage. Once the operating envelope of the aircraft is available the equilibrium points can be chosen on the envelope boundaries and inside the envelope to design the linear controllers. This section explains the process in determining the envelope of the general aviation aircraft used in this thesis.

The range of velocities at which an aircraft can fly is limited on the lower end by the stall speed and at the higher end by the maximum power available. The upper end of the altitude to which an aircraft can fly is limited by the power available at a particular altitude.

The stall speed of an aircraft is defined as the minimum airspeed at which one can maintain straight level flight. The stalling speed of an aircraft depends on the maximum lift coefficient of the aircraft $C_{L, \max }$. The stall velocity of the aircraft is given by

$$
V_{\text {Stall }}=\sqrt{\frac{2 W}{\rho_{\infty} S C_{L, \max }}}
$$

where $W$ is the weight of the aircraft, $S$ is the wing reference area, $\rho_{\infty}$ is the density of ambient air and $C_{L, \text { max }}$ is the maximum lift coefficient of the aircraft. The stall velocity changes with altitude as the density of air decreases with altitude. The stall velocity therefore increases as the aircraft flies at higher altitude. Since the stall velocity is the minimum velocity at which the aircraft can maintain steady level flight it forms the low speed end curve in the flight envelope.

At the high end the maximum velocity at which an aircraft can fly is determined by the maximum power available. As an aircraft flies at higher velocities, the drag of the aircraft increases and to balance this in steady level flight an equivalent amount of thrust is necessary. 
The thrust available for a given engine depends on the engine itself as well as the altitude at which the aircraft is flying. In the present context the data for actual engine thrust variation with altitude was not available directly. In order to compute the max velocity at which the aircraft could fly at a given altitude the rate of climb performance of the general aviation aircraft at various airspeeds, was obtained from the pilot's operating handbook.

The rate of climb of an aircraft at a given velocity $V_{\infty}$ is given by

$$
\text { Rate of climb }=\frac{T_{A} V_{\infty}-D V_{\infty}}{W}
$$

Where $T_{A}$ the maximum available thrust of the aircraft, $D$ is the total drag and $V_{\infty}$ is the airspeed or velocity of the aircraft. The numerator in the rate of climb equation is the difference between the power available, $P_{A}=T_{A} V_{\infty}$, and the power required, $P_{R}=T_{R} V_{\infty}$, to fly level at a given velocity. We can calculate the power required to fly at the velocity for which the rate of climb of the aircraft is known, from this we can calculate the total power available at a given altitude. Once we have the total available power at a given altitude we can calculate the maximum velocity at which the aircraft can fly at that particular altitude solving the equation.

$$
\frac{C_{D_{0}} \rho S}{2} V_{\max }{ }^{4}+\frac{2 W^{2}}{\pi e A R \rho S}=P_{R} V_{\infty}=P_{A} V_{\max }
$$

and taking the maximum value of the real roots.

In the design of the nonlinear controller presented in this thesis, the lower end of the design region $I R$ is based not on the stall speed at a given altitude, but on the airspeed for level flight of the aircraft at minimum thrust. The reason for this is to avoid flying the aircraft on the backside of the power curve where a decrease in velocity $V_{\infty}$ requires an increase in power. The airspeed at minimum thrust for a reciprocating engine and propeller configuration is given by [26]. 


$$
V_{\min T}=\sqrt{\frac{2 W}{\rho S} \sqrt{\frac{\pi e A R}{C_{D_{0}}}}}
$$

where $W$ is the weight of the aircraft, $S$ the wing reference area, $\rho$ is the density of air, $e$ is the oswalds efficiency factor, $A R$ is the aspect ratio of the wing, $C_{D_{0}}$ is the drag at zero lift.

Once the envelope bounded by the airspeed at minimum thrust on the lower end and the maximum velocity at the higher end at different altitudes is known, we can now choose the operating (design) points within and on the boundary of the envelope. A total of thirty six operating points were chosen as shown in Figure 9. The small perturbation model about these steady state operating points are obtained and used for designing the linear controllers.

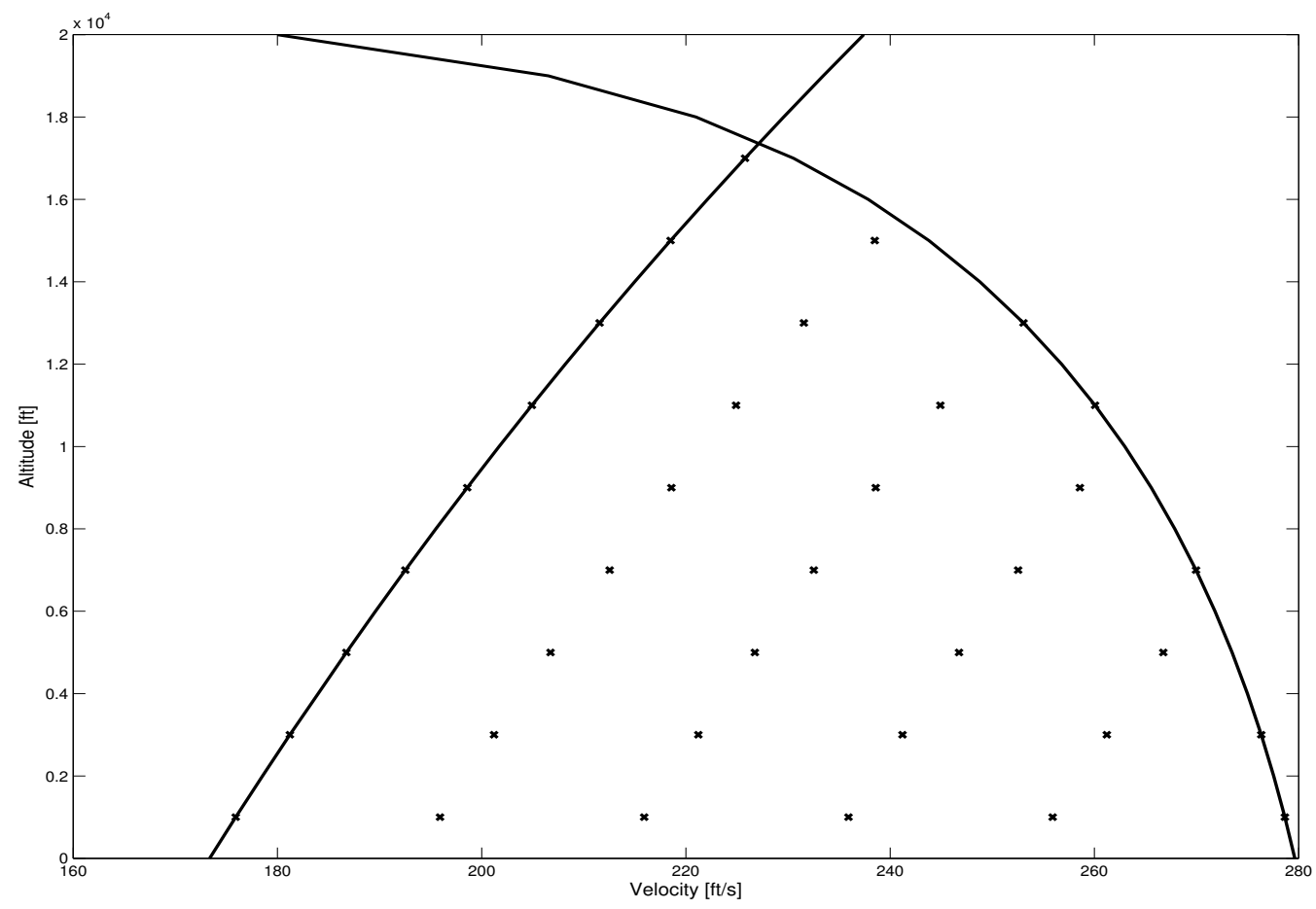

Figure 9: Flight envelope 


\subsection{Linear controller design}

At each of the thirty six operating points considered in the flight envelope of the general aviation aircraft, linear controllers are designed such that they meet required system specification. These controllers provide the performance baseline for the nonlinear neural network controller. The three longitudinal equation of the aircraft are linearized at different operating points based on the scheduling vector $a$. A small perturbation model of the nonlinear equation is obtained at each and every operating point of the form

$$
\Delta \dot{x}=F \Delta x(t)+G \Delta u(t)
$$

The matrices $F$ and $G$ are linear and time invariant. As this process is carried at each and every operating point it results in a set of thirty $\operatorname{six} F$ and $G$ matrices indexed by $k=1,2 \ldots . .36$. Linear control theory is used to design controllers using the $F$ and $G$ matrices at each operating point.

The perturbation model for the longitudinal model is a fourth order linear differential equation of the form given below

$$
\Delta \dot{x}=F_{L} \Delta x(t)+G_{L} \Delta u(t)
$$

where the longitudinal state matrix is $x=\left[\begin{array}{llll}V & \gamma & q & \theta\end{array}\right]^{T}$ and the control matrix is $u=\left[\begin{array}{ll}\delta_{T} & \delta_{E}\end{array}\right]^{T}$. The linear controllers designed for the set of linear models are used to initialize the feedback, command-integral, which are combined to form the action network, and critic neural networks.

The linear controllers to be designed are based on a Proportional-Integral (PI) linear control structure. A PI controller shown in Figure 10 can be designed to modify the stability of the system and the transient response to meet performance specifications. The PI controller achieves this using the feedback gain matrix $C_{B}$, the system response is guaranteed to be a type-1 
response [23] to command inputs through the proportional gain matrix $C_{F}$, and the command integral gain matrix $C_{I}$. The linearized output equations about the nominal conditions is given by

$$
\Delta y_{s}(t)=H_{x} \Delta x(t)+H_{u} \Delta u(t)
$$

The objectives of the control system are evaluated based on the performance measure of the form

$$
\begin{gathered}
J=\lim _{t_{f} \rightarrow \infty} \frac{1}{2} \int_{0}^{t_{f}} L\left[x_{a}, \tilde{u}, \tau\right] d \tau \\
=\lim _{t_{f} \rightarrow \infty} \frac{1}{2} \int_{0}^{t_{f}}\left[x_{a}^{T}(\tau) Q_{a} x_{a}(\tau)+2 x_{a}(\tau) M_{a} \tilde{u}(\tau)+\tilde{u}^{T}(\tau) R_{a} \tilde{u}(\tau)\right] d \tau
\end{gathered}
$$

which is minimized with respect to $\tilde{u}$. $x_{a}$ represents an augmented state that is given by $x_{a} \equiv\left[\tilde{x}^{T} \xi^{T}\right]^{T}$ where

$$
\tilde{x} \equiv\left(x-x_{0}\right)-\left(x_{c}-x_{0}\right)=x-x_{c}
$$

is the error between the actual state $x$ and the commanded state $x_{c}$ and $x_{0}$ is the equilibrium state. $\xi$ is the time integral of the output error.

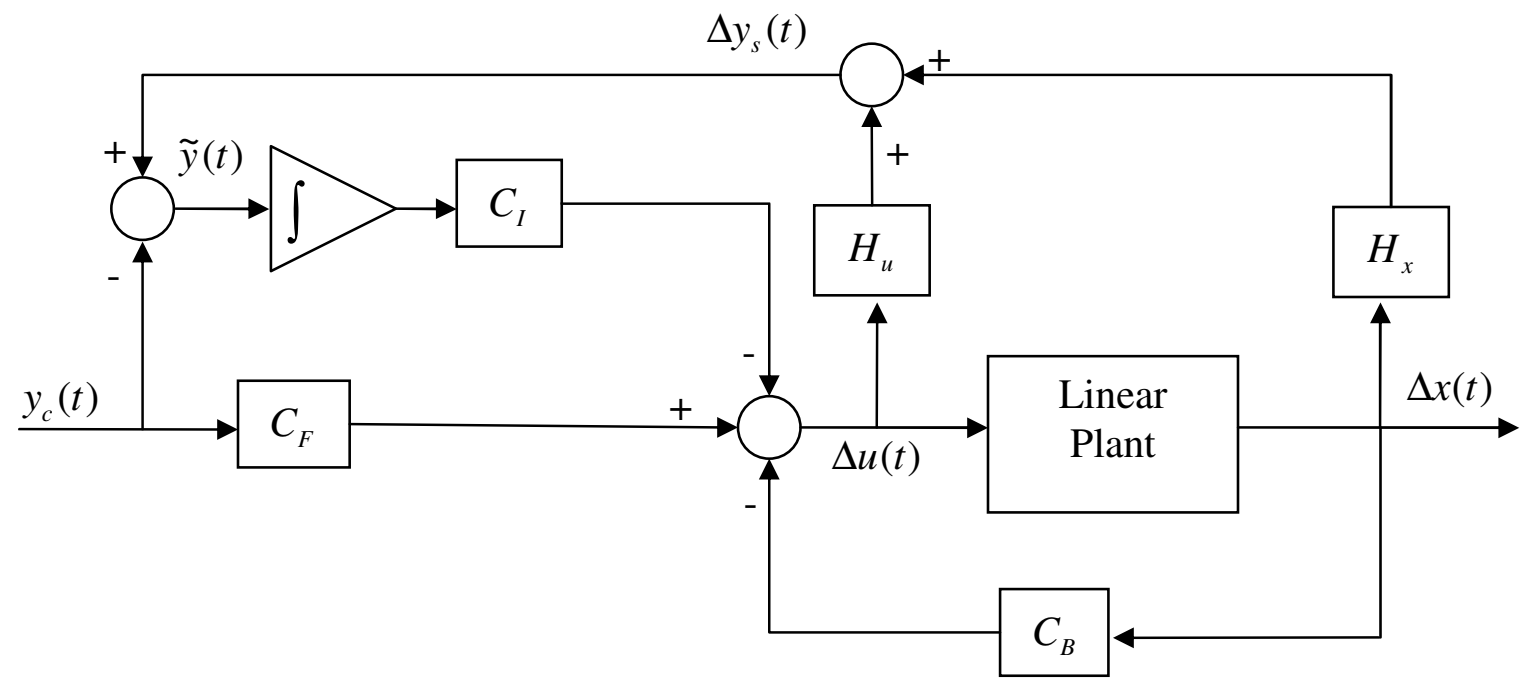

Figure 10: PI controller 
The output error and the minimizing control are also defined as the perturbation from the set point $\left[V_{C} \quad \gamma_{C}\right]$ commanded by the pilot, i.e. $\tilde{y} \equiv y_{s}-y_{C}$ and $\tilde{u} \equiv u-u_{c}$. The optimal control deviation to minimize the performance measure can be shown to be given by [23].

$$
\tilde{u}(t)=-C_{a} x_{a}(t)=-\left[\begin{array}{ll}
C_{B} & C_{I}
\end{array}\right]\left[\begin{array}{rl}
\tilde{x}(t) & \xi(t)
\end{array}\right]^{T}
$$

The control gain matrix $C_{a}$, is partitioned into the two matrices $C_{B}$ and $C_{I}$

$$
\tilde{u}(t)=-C_{B} \tilde{x}(t)-C_{I} \xi(t)
$$

The proportional gain matrix $C_{F}$ is obtained from the feedback gain matrix and set point matrices[16].

$$
C_{F}=B_{22}+C_{B} B_{12}
$$

For the linearized system, the set point is defined by letting the state-rate perturbation $\Delta \dot{x}$ equal to zero. This gives the steady state flight condition

$$
\left[\begin{array}{c}
0 \\
y_{C}
\end{array}\right]=\left[\begin{array}{cc}
F & G \\
H_{x} & H_{u}
\end{array}\right]\left[\begin{array}{l}
\Delta x_{c} \\
\Delta u_{c}
\end{array}\right]
$$

The set point state and control perturbations are obtained from the command perturbation as given

$$
\left[\begin{array}{l}
\Delta x_{c} \\
\Delta u_{c}
\end{array}\right]=\left[\begin{array}{cc}
F & G \\
H_{x} & H_{u}
\end{array}\right]^{-1}\left[\begin{array}{c}
0 \\
\Delta y_{C}
\end{array}\right] \equiv\left[\begin{array}{ll}
B_{11} & B_{12} \\
B_{21} & B_{22}
\end{array}\right]\left[\begin{array}{c}
0 \\
\Delta y_{C}
\end{array}\right]=\left[\begin{array}{l}
B_{12} \Delta y_{C} \\
B_{22} \Delta y_{C}
\end{array}\right]
$$

The Riccati matrix, $P_{a}$, needed to train the critic network is obtained by solving the matrix Riccati equation given in equation (31) constructed in terms of the augmented state and control deviation. The weighting matrices in the performance measure are formulated using implicit model following as explained in the next section. 


\subsection{Implicit model following}

In formulating the weighting matrices $Q, M, R$ in equation (80) in the performance measure we use implicit model following to induce the closed loop system to follow the response of an ideal model that meets performance specifications. The ideal model which the system has to follow is a linear model of the same order as the linearized longitudinal model of the aircraft. The selection of an ideal model for longitudinal control of an aircraft is based on handling quality requirements that meet either civilian or military specifications for different aircraft types and phases of flight.

The general aviation aircraft for which the nonlinear neural controller is designed is classified as a class I aircraft. The longitudinal flying qualities of the aircraft are specified by imposing boundaries on the damping ratio of the two modes, phugoid and short period, which characterize the longitudinal response of the aircraft. The boundaries for the damping ratio vary based on the flight phase. Accurate following of the pilot input commands are necessary during the terminal flight phase conditions like takeoff and landing. The boundaries of the damping ratio of the short period and phugoid mode for the terminal flight phase for a class I aircraft are given in Table 1 [24]

\begin{tabular}{|c|}
\hline Longitudinal flying qualities \\
\hline \hline Phugoid mode \\
\hline$\zeta_{p}>0.04$ \\
\hline Short-period mode \\
\hline $0.35 \leq \zeta_{s p} \leq 1.30$ \\
\hline
\end{tabular}

Table 1: Flying qualities for class I aircraft in terminal phase flight 
The ideal model of the longitudinal motion of the aircraft using the stability derivatives with the state perturbation $\Delta x=\left[\begin{array}{llll}\Delta V & \Delta \gamma & \Delta q & \Delta \alpha\end{array}\right]^{T}$ is given by [25]

$$
F_{m}=\left[\begin{array}{cccc}
T D_{v} & -g \cos \gamma_{0} & T D_{q} & T D_{\alpha} \\
L_{v} / V_{0} & \left(g / V_{0}\right) \sin \gamma_{0} & L_{q} / V_{0} & L_{\alpha} / V_{0} \\
M_{v} & 0 & M_{q} & M_{\alpha} \\
-L_{v} / V_{0} & -\left(g / V_{0}\right) \sin \gamma_{0} & \left(1-L_{q} / V_{0}\right) & -L_{\alpha} / V_{0}
\end{array}\right]
$$

The above matrix $F_{m}$ for the ideal aircraft which is in approximate block diagonal form and can be partitioned into the phugoid parameters and short period parameters.

The phugoid damping ratio and the short period can be determined by the following formulae

$$
\begin{gathered}
\omega_{n p} \approx \sqrt{\left(g L_{v} / V_{0}\right)} \\
\varsigma_{p} \approx-T D_{v} / 2 \omega_{n p} \\
\omega_{n s p} \approx \sqrt{\left(-M_{\alpha}\left(1-L_{q} / V_{0}\right)-M_{q} L_{\alpha} / V_{0}\right)} \\
\varsigma_{s p} \approx\left(L_{\alpha} / V_{0}-M_{q}\right) / 2 \omega_{n s p}
\end{gathered}
$$

The matrix $F$ for the ideal model can be computed based on the flying qualities to be met by the actual aircraft. As there are more parameters than the number of equations, certain values can be assumed to be equal to the actual aircraft. In this thesis we equated the $L_{q} / V_{0}, T D_{q}, M_{v}, M_{\alpha}$, $L_{\alpha} / V_{0}, T D_{\alpha}$ and $L_{v} / V_{0}$ to be equal to the median value of the thirty six linearized models obtained at each operating point. The next step is to solve the equations obtaining the desired values of the natural frequency and damping ratio. 
The desired value of the damping ratio for the phugoid mode and the short period mode of the ideal model were selected to be $\varsigma_{p} \approx 0.1$ and $\varsigma_{s p} \approx 1$. These values were then used to determine the parameters $T D_{v}$ and $M_{q}$ using equations (89) and (91).

The ideal model obtained needs to be transformed into the same state vector as the system to be controlled. In this thesis we are designing for pure longitudinal motion for which the relationship between the flight path angle $\gamma$, the angle of attack $\alpha$ and the pitch angle $\theta$ is given by

$$
\theta=\gamma+\alpha
$$

Now the transformation matrix $T$ is given by

$$
T=\left[\begin{array}{llll}
1 & 0 & 0 & 0 \\
0 & 1 & 0 & 0 \\
0 & 0 & 1 & 0 \\
0 & 1 & 0 & 1
\end{array}\right]
$$

The transformation from the state $\Delta x=\left[\begin{array}{llll}\Delta V & \Delta \gamma & \Delta q & \Delta \alpha\end{array}\right]^{T}$ to $\Delta x_{m}=\left[\begin{array}{llll}\Delta V & \Delta \gamma & \Delta q & \Delta \theta\end{array}\right]^{T}$ is given by

$$
\begin{gathered}
{\left[\begin{array}{c}
\Delta V \\
\Delta \gamma \\
\Delta q \\
\Delta \theta
\end{array}\right]=\left(\left[\begin{array}{llll}
1 & 0 & 0 & 0 \\
0 & 1 & 0 & 0 \\
0 & 0 & 1 & 0 \\
0 & 1 & 0 & 1
\end{array}\right]\left[\begin{array}{cccc}
T D_{v} & -g \cos \gamma_{0} & T D_{q} & T D_{\alpha} \\
L_{v} / V_{0} & \left(g / V_{0}\right) \sin \gamma_{0} & L_{q} / V_{0} & L_{\alpha} / V_{0} \\
M_{v} & 0 & M_{q} & M_{\alpha} \\
-L_{v} / V_{0} & -\left(g / V_{0}\right) \sin \gamma_{0} & \left(1-L_{q} / V_{0}\right) & -L_{\alpha} / V_{0}
\end{array}\right]\left[\begin{array}{cccc}
1 & 0 & 0 & 0 \\
0 & 1 & 0 & 0 \\
0 & 0 & 1 & 0 \\
0 & 1 & 0 & 1
\end{array}\right]^{-1}\right)\left[\begin{array}{c}
\Delta V \\
\Delta \gamma \\
\Delta q \\
\Delta \alpha
\end{array}\right]} \\
{\left[\begin{array}{c}
\Delta V \\
\Delta \gamma \\
\Delta q \\
\Delta \theta
\end{array}\right]=\left[F_{m}\right]\left[\begin{array}{c}
\Delta V \\
\Delta \gamma \\
\Delta q \\
\Delta \alpha
\end{array}\right]}
\end{gathered}
$$

Now the state equation of the ideal model is given as 


$$
\Delta \dot{x}_{m}=F_{m} \Delta x_{m}(t)
$$

In implicit model following the state rate of the actual system should be the same as that of the ideal model. Since we need the actual system states to follow the ideal model states, the performance measure or cost function to be minimized can be given by the following

$$
J=\lim _{t_{f} \rightarrow \infty} \frac{1}{2} \int_{t_{0}}^{t_{f}}\left\{\left(\Delta \dot{x}(\tau)-\Delta \dot{x}_{m}(\tau)\right)^{T} Q_{m}\left(\Delta \dot{x}(\tau)-\Delta \dot{x}_{m}(\tau)\right)\right\} d \tau
$$

This is equal to

$$
J=\frac{1}{2} \int_{0}^{t_{f}}\left[\Delta x^{T}(\tau) Q \Delta x(\tau)+2 \Delta x^{T} M \Delta u(\tau)+\Delta u^{T}(\tau) R \Delta u(\tau)\right] d \tau
$$

when the weighting matrices $Q, M, R$ are given by equations (99),(100) and (101) respectively

$$
\begin{gathered}
Q=\left(F-F_{m}\right)^{T} Q_{m}\left(F-F_{m}\right) \\
M=\left(F-F_{m}\right)^{T} Q_{m} G \\
R=G^{T} Q_{m} G+R_{0}
\end{gathered}
$$

The matrices $G$ and $F$ are the control and system matrix from the perturbation model equation 76 is obtained at each operating point. The matrix $R_{0}$ is a constant matrix which represents the separate cost of control. The actual system follows the ideal model when the following condition is satisfied [23].

$$
\left(G G^{P I}-I_{n}\right)\left(F-F_{m}\right)=0
$$

Since the actual system model $F$ is not exactly equal to the ideal model $F_{m}$ perfect model following cannot be achieved. The weighting matrices $Q_{m}$ and $R_{0}$ can now be chosen such that the actual response that follows the ideal model response for the pilot input commands of flight path angle and velocity with reasonable amount of control input. 
In order to achieve a type one response, the integral of the error between the commanded velocity and flight path angle needs to be considered in the cost function. We need to augment the states with the error integral $\xi$ leading to the cost function given by

$$
J=\frac{1}{2} \int_{0}^{t_{f}}\left[\Delta x_{a}^{T}(\tau) Q_{a} \Delta x_{a}(\tau)+2 \Delta x_{a}^{T} M_{a} \Delta u(\tau)+\Delta u^{T}(\tau) R_{a} \Delta u(\tau)\right] d \tau
$$

where $\Delta x_{a}=\left[\begin{array}{ll}\Delta x & \xi\end{array}\right]^{T}$ is the state augmented by the error integral and the matrices $Q_{a}$ and $M_{a}$ are given by

$$
\begin{aligned}
& Q_{a}=\left[\begin{array}{ll}
Q & 0 \\
0 & Q_{\xi}
\end{array}\right] \\
& M_{a}=\left[\begin{array}{c}
M \\
0
\end{array}\right] \\
& R_{a}=R
\end{aligned}
$$

The output equation for the linearized model is given by

$$
y(t)=\left[\begin{array}{llll}
1 & 0 & 0 & 0 \\
0 & 1 & 0 & 0
\end{array}\right] \Delta x_{a}(t)+[0] \Delta u(t)
$$

Now the PI gains for all the thirty six models are calculated by solving the Riccati equations of the form given in equation (31) using the weighting matrices $Q_{a}, M_{a}$ and $R_{a}$ resulting in thirty six control gain matrices $\{C\}^{1 \ldots \ldots .36}$ and Riccati matrices $\{P\}^{1,2 \ldots 36}$. The gain matrices $\{C\}^{1 \ldots \ldots .36}$ are used to obtain the set of feedback matrices $\left\{C_{B}\right\}^{1,2 \ldots 36}$, the set of integral matrices $\left\{C_{1}\right\}^{1,2 \ldots 36}$ and the forward gain matrices $\left\{C_{F}\right\}^{1,2 \ldots 36}$ of Figure 10 using equations (83) and (84). 


\subsection{Neural network based controller}

The next step after the design of the linear controllers is to replace the gain matrices with neural networks. Each of the feedback matrices, the integral matrices are replaced with a neural network and are initialized such that they meet performance specifications of the linear controller they replace, These two networks are combined to form the action network. The neural network based controller is as shown in Figure 11.

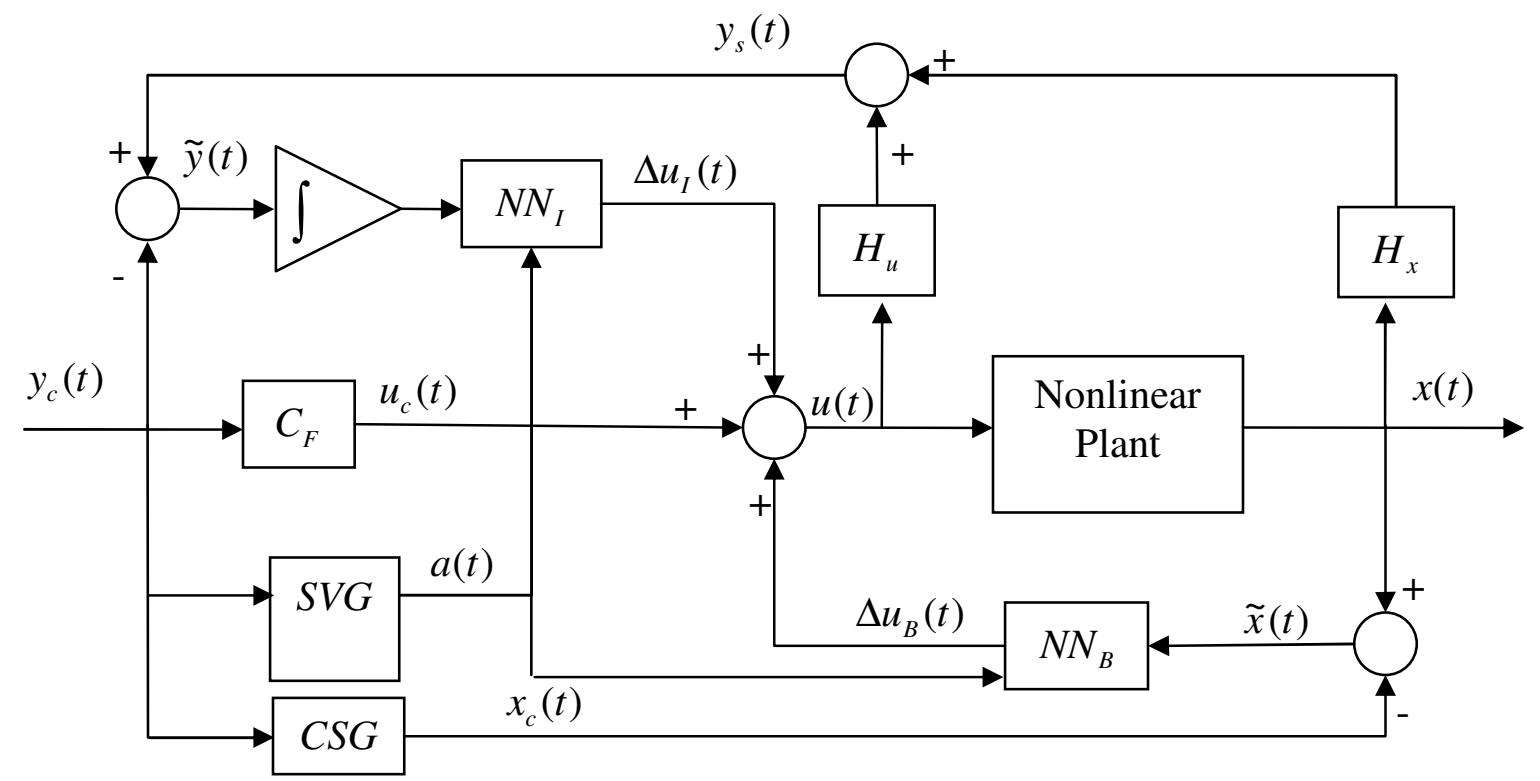

Figure 11: Neural network based PI controller

As seen from Figure 11, the input output structure is unchanged from the linear controller. The output $y(t)$ and the set point $\left(x_{c}, u_{c}\right)$ are computed for the nonlinear system. The scheduling variable generator produces the scheduling variable inputs to the neural networks. The command state generator uses the kinematic equations to generate the other elements of the state such that they are compatible with the pilot input command of velocity and flight path angle. The two gain 
matrices, feedback gain matrix $C_{B}$, and the integral matrix $C_{I}$ are replaced by the neural networks designated as $N N_{B}$ and $N N_{I}$ respectively. The forward matrix $C_{F}$, which is used to calculate the commanded state $x_{c}$ and the commanded $\delta_{T}$ and $\delta_{E}$,can also be replaced by an equivalent neural network. But in this thesis the forward gain matrix is retained from the linear design.

The inputs to all the neural networks consist of the scheduling variables $a=\left[\begin{array}{lll}V(t) & H(t)\end{array}\right]^{T}$. In addition the neural networks $N N_{B}$ and $N N_{I}$ are the state deviation $\tilde{x}(t)$ and the command-error integral $\xi(t)$ respectively. The total control signal is the addition of the output from the forward gain matrix, the output of the feedback neural network and the output of the integral neural network, i.e.

$$
u(t)=u_{c}(t)+\Delta u_{B}(t)+\Delta u_{I}(t)
$$

The next step in the design of the neural network based nonlinear controller is to determine the architecture and the initial weights of the neural network. The methodology presented in chapter three is used now to determine the initial weights and architecture of the feedback neural network and the integral neural network.

At the operating points considered, the output of the feedback neural network must be zero. This is true because at the operating point the state $x(t)$ approaches the commanded state $x_{c}(t)$ and $\tilde{x}(t)$ tends to zero. Therefore

$$
z_{B}[\tilde{x}(t), a(t)]_{K}=z_{B}[0, a(t)]_{K}=0
$$

The control deviation $\tilde{u}(t)$ is the difference between the commanded input $u_{c}(t)$ generated by the forward matrix and the sum of the control inputs generated by the feedback neural network and the integral neural network, i.e.

$$
\tilde{u}(t)=\Delta u_{B}(t)+\Delta u_{I}(t)=z_{B}(t)+z_{I}(t)
$$


Differentiating the above equation with the state deviation at the operating point we get

$$
\left(\frac{\partial z_{B}(t)}{\partial \tilde{x}(t)}\right)^{K}=\left(\frac{\partial \tilde{u}}{\partial \tilde{x}(t)}\right)^{K}=\left(-C_{B}\right)^{K}
$$

Similarly the output of the integral neural network is zero when the command error integrals are zero, i.e.

$$
z_{I}[\xi(t), a(t)]_{K}=z_{I}[0, a(t)]_{K}=0
$$

and the derivative of the integral neural network with respect to the command error integral is given by

$$
\left(\frac{\partial z_{I}(t)}{\partial \xi(t)}\right)^{K}=\left(\frac{\partial \tilde{u}}{\partial \xi(t)}\right)^{K}=\left(-C_{I}\right)^{K}
$$

Now the training set for the feedback neural network is a set of thirty six input output pairs of the form $\left\{\left[\begin{array}{ll}0 & a^{K^{T}}\end{array}\right]^{T}, 0, C_{B}{ }^{K}\right\}$ for the thirty six operating points considered. Similarly the training set for the integral neural network is of the form $\left\{\left[\begin{array}{ll}0 & a^{K^{T}}\end{array}\right]^{T}, 0, C_{I}^{K}\right\}$. Initially the neural networks for each of the control signal, i.e. $\delta_{T}$ and $\delta_{E}$, is trained separately by only considering the corresponding rows of the gradient matrices $C_{B}$ and $C_{I}$, in the next chapter these are combined and used to generate the total control deviation signals.

Now the gradient based procedure explained in chapter three is used to initialize both the feedback and the integral neural network. Both the neural networks are composed of single hidden layer sigmoidal neural networks with the number of neurons equal to the number of the training pairs. One important feature of the neural network is that the direct contribution of the scheduling variables to the output of the neural network is subtracted using a mirror image of the initialized network. This is done to eliminate any bias the scheduling variables might produce away from the nominal condition. This is done to prevent the neural network from adapting for a 
change in the scheduling variables of $V$ and $h$. The final architecture for the initialized feedback neural network for one of the control signals is shown in Figure 12 and for the integral neural network is shown in Figure 13.

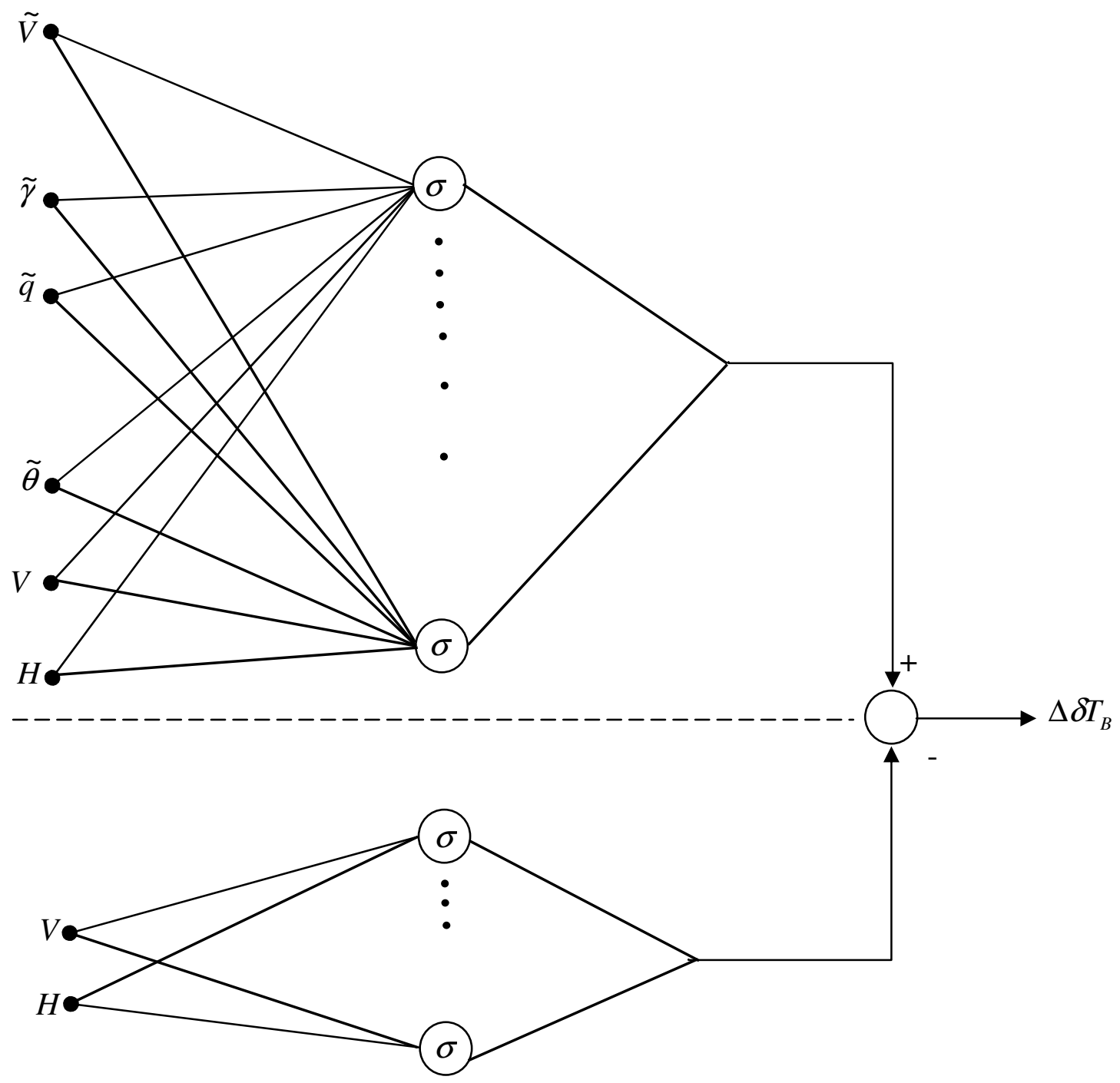

Figure 12: Initialized feedback neural network architecture 


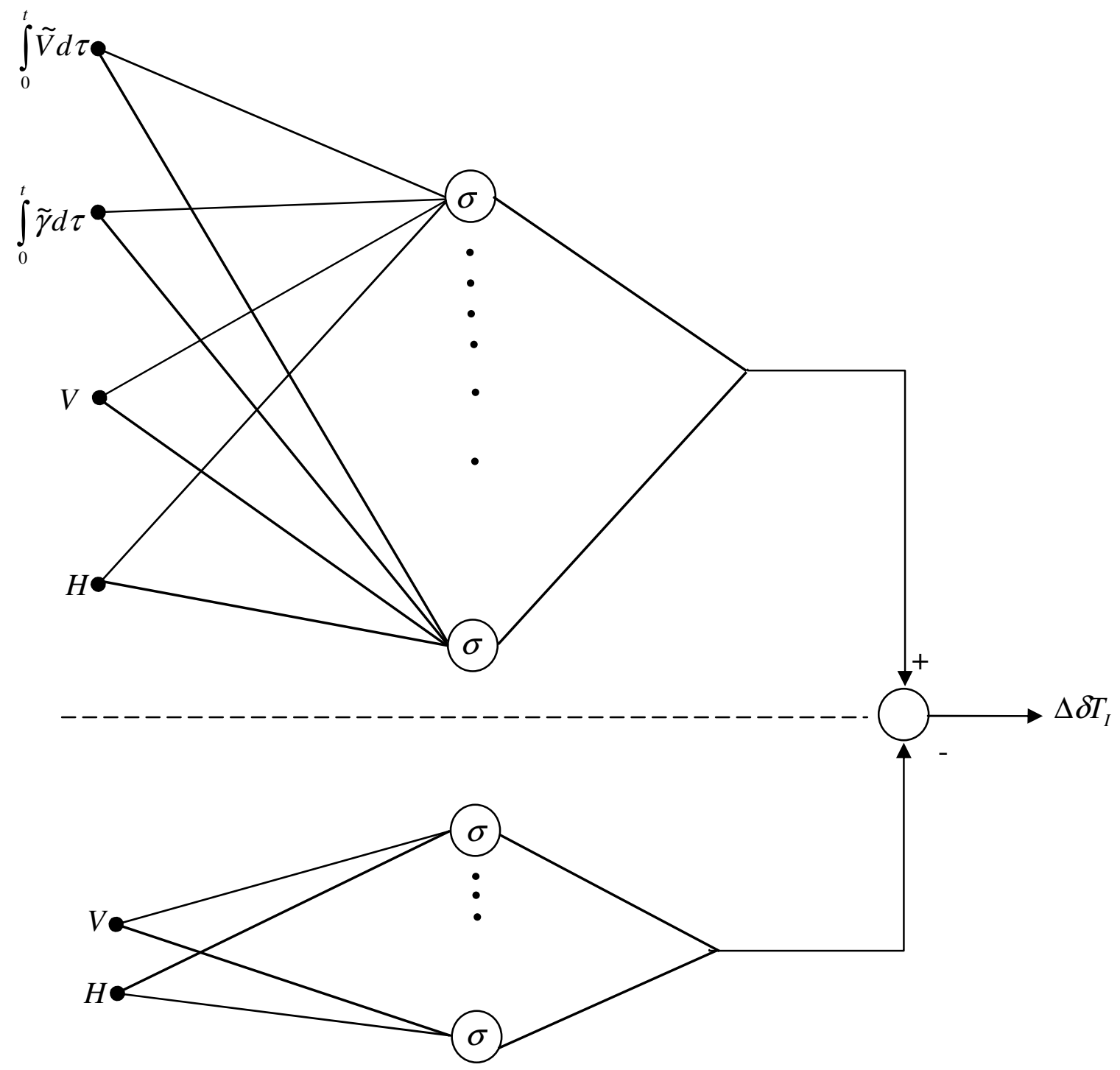

Figure 13: Initialized integral neural network architecture

\subsection{Response of initialized neural network}

The initialized neural network are now tested and evaluated with respect to the linear controller that they replace. The neural network controller is tested on the longitudinal model of the aircraft. The neural network controller response is then compared to the linear controller response designed for the operating point considered. In order to test the generalization properties of the neural network, the response of the neural network controller is also evaluated 
at a point other than the operating point called the interpolation point. In order to compare the performance of the neural network controller a linear controller is designed for the interpolation point considered but not used in initializing the neural network.

Case 1: Response at a design point

The neural network controller response is evaluated at the design point $\left(V_{0}, h_{0}\right) \equiv(211.74,5000)$. The response of the controller to a two degree step command in the flight path angle and three feet per second step increase in the velocity is considered. Figure 14 shows the variation of the flight path angle and velocity.
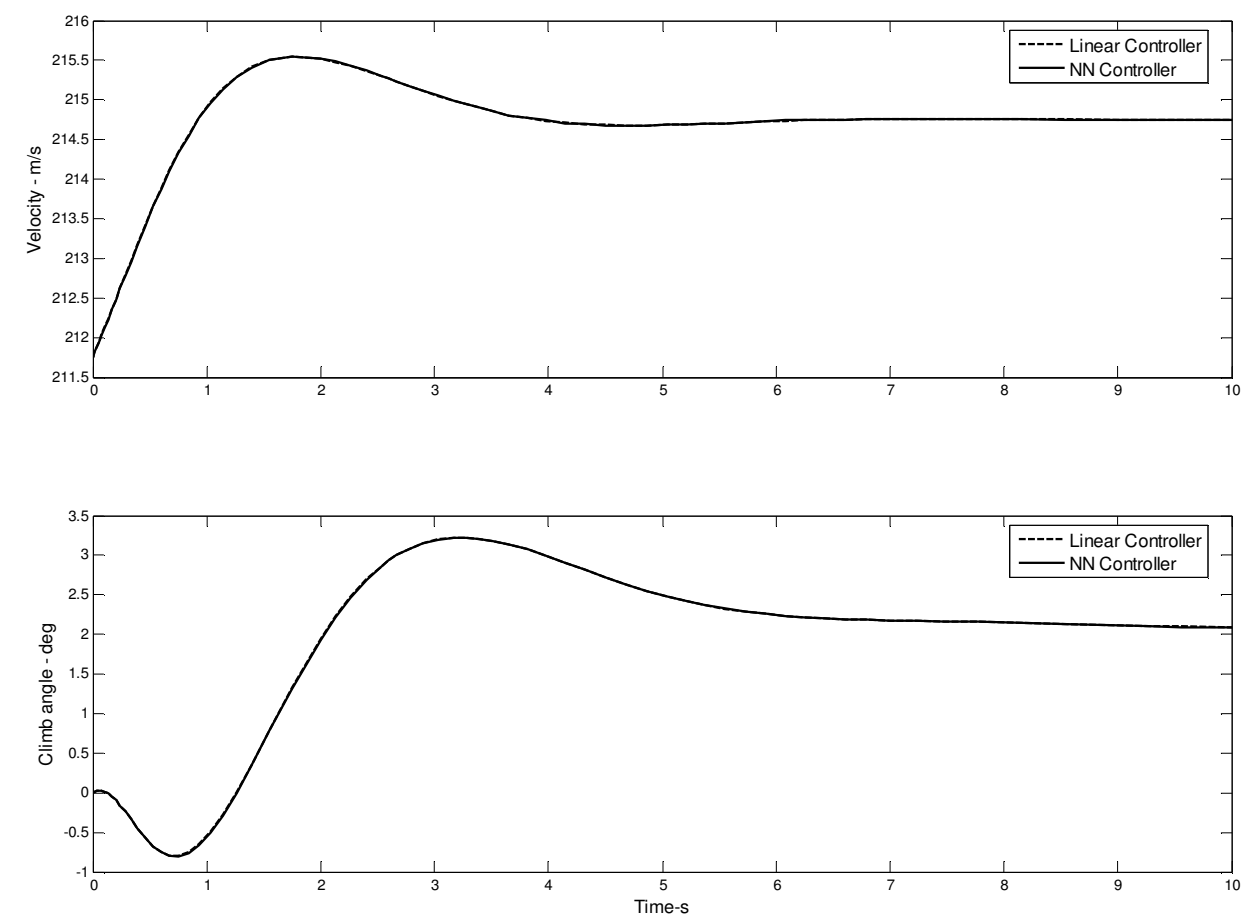

Figure 14: Response at a design point

Case 2: Response at an interpolation point

The neural network controller response is evaluated at the Interpolation point $\left(V_{0}, h_{0}\right) \equiv(209,4000)$. The response of the controller to a two degree step command in the flight 
path angle and three feet per second step increase in the velocity is considered. Figure 15 shows the variation of the flight path angle and velocity.
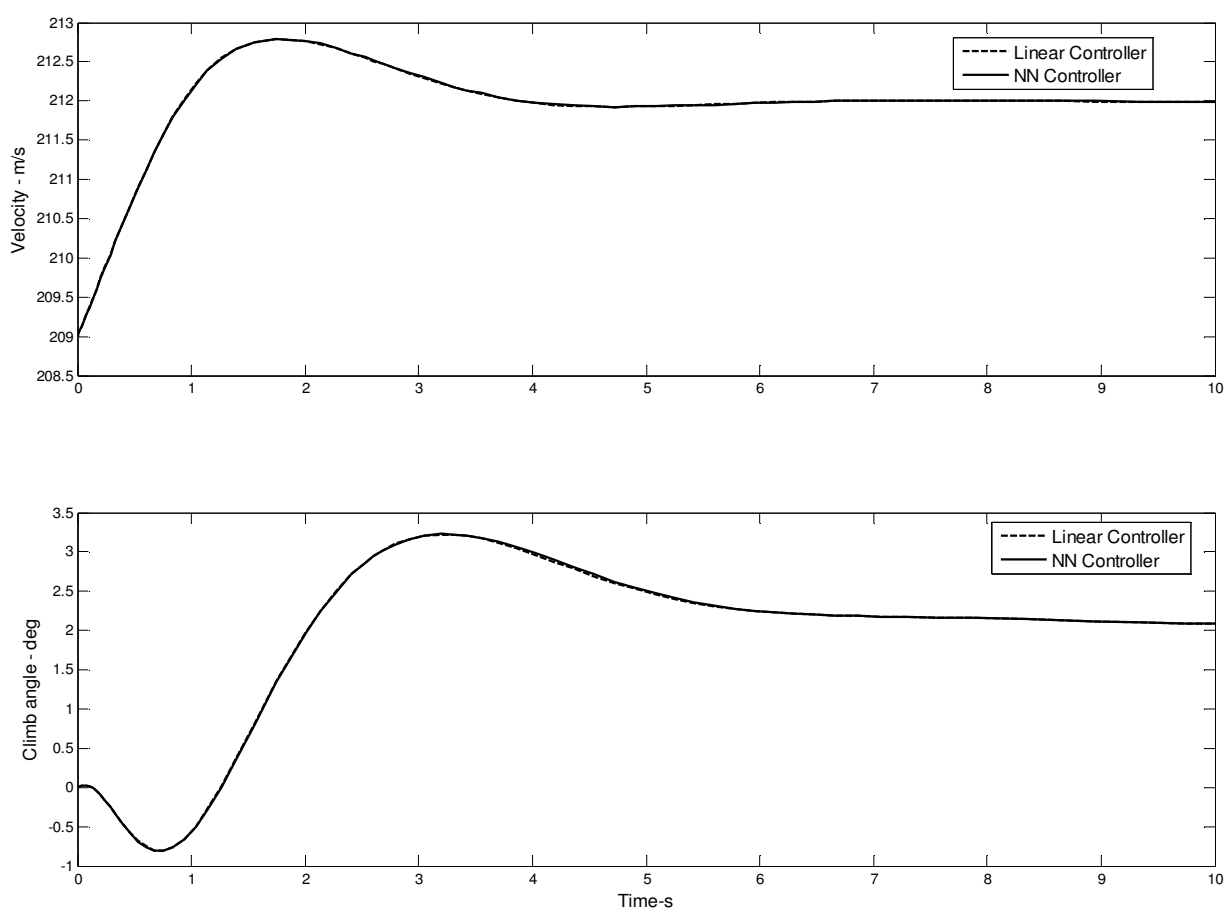

Figure 15: Response at an interpolation point

\subsection{Critic neural network}

The critic neural network, $N N_{C}$, is used to evaluate the performance of the action network online with the dual heuristic programming adaptive critic architecture. The critic evaluates the performance by approximating the derivative of the cost to go or value function. The critic network is also initialized as the other two neural networks, the feedback neural network and the integral neural network.

The critic neural network is not used in the testing of the initialized controller as the weights of the feedback and the integral neural network are not adapted. In the online phase the weights of the action network, which is obtained by combining the feedback and integral neural 
network, is adapted to improve performance in the presence of nonlinearities as well as control failures. The critic neural network optimizes the same cost function that is optimized in the pretraining phase as described in section 2.4. The critic network approximates the derivative of the value function given by

$$
\lambda_{a}[x(t)] \equiv \frac{\partial V\left(x_{a}, t\right)}{\partial x_{a}(t)}
$$

Thus the output of the critic neural network is given by

$$
\lambda_{a}(t)=z_{C}\left[x_{a}(t), a\right]
$$

For the set of operating points considered in the linear controller design, the state deviation $\tilde{x}(t)$ and the command error integral $\xi(t)$ are equal to zero. Therefore the output of the critic neural control at the operating point is zero, i.e.

$$
z_{C}\left(\left[x_{a}(t), a(t)\right]\right)^{K}=\left(z_{C}[0, a]\right)^{K}=0
$$

The gradient of the critic neural network with respect to the augmented state is given by

$$
\frac{\partial z_{C}(t)}{\partial x_{a}(t)}=\frac{\partial \lambda_{a}(t)}{\partial x_{a}(t)}
$$

At the operating points considered the derivative of the value function with respect to the augmented state is given by

$$
\left(\frac{\partial V\left(x_{a}, t\right)}{\partial x_{a}(t)}\right)^{k}=x_{a}^{T}(t) P_{a}^{k}
$$

Therefore the gradient of the critic neural network must satisfy the condition

$$
\left(\frac{\partial z_{C}(t)}{\partial x_{a}(t)}\right)^{k}=\left(\frac{\partial \lambda_{a}(t)}{\partial x_{a}(t)}\right)^{k}=P_{a}^{k}
$$


Now a training set of the form $\left\{\left[\begin{array}{ll}0 & a^{K^{T}}\end{array}\right]^{T}, 0, P_{a}{ }^{K}\right\}$ similar to the feedback and integral neural network training sets is available. The initialization can be now done based on the algebraic training explained in chapter three. As before six different neural networks are initialized separately and combined as explained in chapter five. The initialized critic neural network for one of the six outputs is as shown in Figure 16.

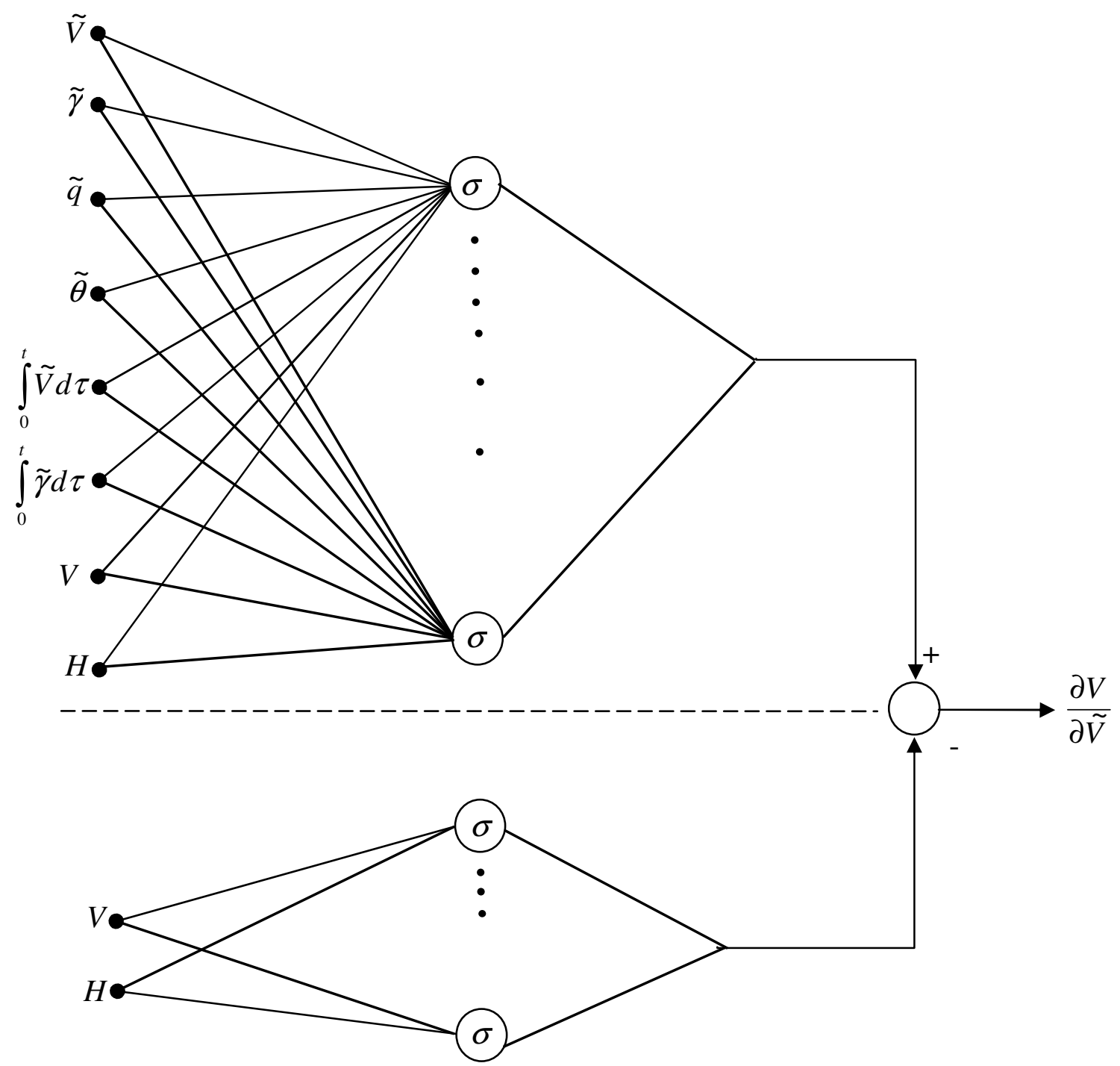

Figure 16: Initialized critic neural network. 


\section{ADAPTIVE CRITIC FLIGHT CONTROL}

In this chapter the neural networks initialized in chapter four are adapted online based on the dual heuristic adaptive critic design to account for nonlinearities not considered in the linear control design. The neural network controller also adapts to control failures as well as parameter variations online. The dual heuristic adaptive critic design is based on the approximate dynamic programming introduced in chapter one. Approximate dynamic programming iterates only on one value of the state and uses these results during the next time step. This eliminates the need for expanding and storing the state vector over time. It has been shown that when the iterative successive approximation concept is used with a parametric structure to predict the cost, the solution converges over time to the optimal trajectory.

Neural networks are the parametric structure of choice as they have properties which lend themselves to approximate functions of multi input multi output space. In dual heuristic dynamic programming the neural networks are used to approximate the gradient of the value function with respect to the state. It has been observed that using the gradient of the cost accelerates the convergence to the optimal trajectory and it alleviates the need for persistence of excitation of the network. In dual heuristic dynamic programming the control law, approximated by the action network, and the gradient of the value function, approximated by the critic network, are adapted based on the recurrence relation of dynamic programming as detailed in section 2.4.

The use of neural networks as function approximators allows for both incremental learning as well as learning in batch mode. As explained in chapter four, the neural networks are intialized in batch mode in the pre training phase using the gains obtained from the linear controller design at the operating point. In the online phase the neural networks learn online to improve system performance incrementally overtime in the presence of nonlinearities as well as control failures 
and modeling errors. One important aspect of the online training is that it preserves the mapping of input and output obtained in the initialization phase. The online training algorithm is based on the dual heuristic programming explained in the next section.

\subsection{Dual heuristic adaptive critic}

The online logic is implemented in discrete time with the action and critic network updating and control action occurring for every time interval $\Delta t$. In the online phase the same metric or cost optimized in the design of the linear controllers is used. This establishes the input output structure of the neural network to be the same as that of the neural networks initialized in the pre training phase. The utility function in discrete time is given by

$$
L_{S D}\left[x_{a}\left(t_{k}\right), \tilde{u}\left(t_{k}\right)\right]=\frac{1}{2}\left[x_{a}^{T}\left(t_{k}\right) Q_{a} x_{a}\left(t_{k}\right)+2 x_{a}\left(t_{k}\right) M_{a} \tilde{u}\left(t_{k}\right)+\tilde{u}^{T}\left(t_{k}\right) R_{a} \tilde{u}\left(t_{k}\right)\right]
$$

where $x_{a}$ is the augmented state $\left[\begin{array}{ll}\tilde{x} & \xi\end{array}\right]^{T}$ and $\tilde{u}$ is the control deviation from the nominal control input. The utility function can also be seen as the cost associated with going from the current state $x_{a}\left(t_{k}\right)$ to the next state $x_{a}\left(t_{k}+1\right)$ which depends on the input control deviation $\tilde{u}\left(t_{k}\right)$. The weighting matrices $Q_{a}, M_{a}$ and $R_{a}$ are the same as used in the linear controller design in the pre training phase.

\subsection{Action and critic networks}

In the pre training phase the neural networks for the integral gain and the feedback gain were trained independently of each other, also the neural networks for the two control inputs $\left[\begin{array}{ll}\delta T & \delta E\end{array}\right]^{T}$ were initialized separately. In the online training phase the neural networks are combined to obtain a single action network which encompasses all the four individual networks initialized in the pre training phase. 
The networks obtained in the pre training phase must be combined to a single network and still meet the performance specifications, i.e. the action network obtained by combining the networks should be equivalent to the individual neural networks. The new network is different from the neural networks in the pre training phase in that they have a vector output as shown in Figure 17 as opposed to a scalar output.

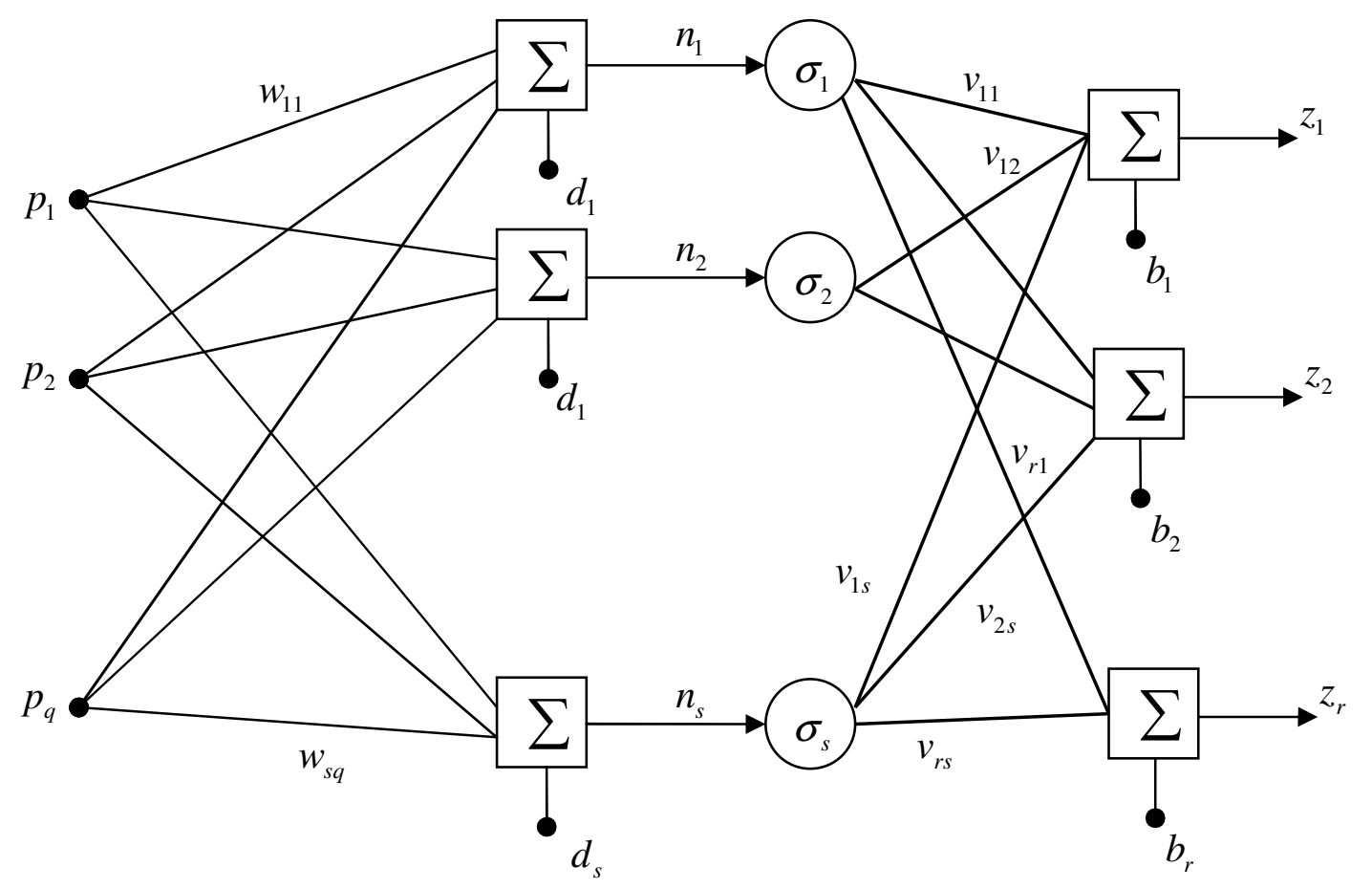

Figure 17: Vector output neural network.

The vector output sigmoidal architecture is used for both the action and critic network in the online training phase. The output of the vector output sigmoidal neural network is given by

$$
z=V \sigma(W p+d)+b
$$


The vector-output neural network notation is equivalent to that of the scalar output neural network. The only difference being that the output weights are now a matrix $V=\left\{v_{l i}\right\}$, where $v_{l i}$ is the weight for the connection between the $i^{\text {th }}$ node and $l^{\text {th }}$ output of the network.

To obtain the vector output sigmoidal neural network for the action network, the four initialized neural network are combined. First the neural networks for the different control inputs are combined, and then the resulting neural networks for the feedback neural network and integral neural network are combined. For combining the two networks for the feedback neural network, it is observed that the inputs to the two neural networks are the same but the outputs are different, one for the throttle and the other for the elevator. It can be shown that the two neural networks can be combined such that they retain all the characteristics of the individual networks. The new neural network has the form as shown in Figure 17. The number of nodes in the combined neural network is equal to the sum of the number of nodes in the individual neural network. The input weights, the output weights and the biases are given by

$$
\begin{aligned}
& W_{B}=\left[\begin{array}{l}
W_{B_{\delta T}} \\
W_{B_{\delta E}}
\end{array}\right] \\
& V_{B}=\left[\begin{array}{cc}
V_{B_{\delta T}} & 0 \\
0 & V_{B_{\delta E}}
\end{array}\right] \\
& d_{B}=\left[\begin{array}{ll}
d_{B_{\delta T}^{T}}^{T} & d_{B_{\delta E}}^{T}
\end{array}\right]^{T}
\end{aligned}
$$

and

$$
b_{B}=\left[\begin{array}{ll}
b_{B_{\delta T}}^{T} & b_{B_{\delta E}}^{T}
\end{array}\right]^{T}
$$

where $W_{B_{\delta I}}$ is the input weight matrix of the feedback network for throttle. $W_{B_{\delta E}}$ is the input weight matrix of feedback network for elevator. $V_{B_{\delta I}}$ and $V_{B_{\delta E}}$ are the output weight vectors of the 
feedback network for throttle and elevator respectively. The input biases of the feedback network for throttle and elevator are $d_{B_{\delta \Gamma}}$ and $d_{B_{\delta \varepsilon}}$. the output biases of the feedback network for throttle and elevator are $b_{B_{\delta r}}$ and $b_{B_{\delta E}}$. The combined feedback neural network performs equivalent to the uncoupled feedback networks that it replaces.

Similarly for the integral neural network the two neural networks can be joined together to obtain a single neural network of the form given above. The combined neural networks weights are given by

$$
\begin{aligned}
& W_{I}=\left[\begin{array}{c}
W_{I_{\delta T}} \\
W_{I_{\delta E}}
\end{array}\right] \\
& V_{I}=\left[\begin{array}{cc}
V_{I_{\delta T}} & 0 \\
0 & V_{I_{\delta E}}
\end{array}\right] \\
& d_{I}=\left[\begin{array}{ll}
d_{I_{\delta T}}^{T} & d_{I_{\delta E}}^{T}
\end{array}\right]^{T}
\end{aligned}
$$

and

$$
b_{I}=\left[\begin{array}{ll}
b_{I_{\delta T}}^{T} & b_{I_{\delta E}}^{T}
\end{array}\right]^{T}
$$

where $W_{I_{\delta T}}$ is the input weight matrix of the Integral network for throttle. $W_{I_{\delta E}}$ is the input weight matrix of feedback network for elevator. The output weight vectors of the feedback network for throttle and elevator respectively are $V_{I_{\delta T}}$ and $V_{I_{\delta 夭}}$. The input biases of the feedback network for throttle and elevator are $d_{I_{\delta r}}$ and $d_{I_{\overparen{ }}}$. The output biases of the feedback network for throttle and elevator are $b_{I_{\delta T}}$ and $b_{I_{\overparen{ }}}$. The combined integral neural network performs equivalent to the uncoupled integral networks that it replaces. 
The next step is to combine the integral neural network and the feedback neural networks into a single neural network which will then be used as the action network. The two vector output sigmoidal networks have the same input but different outputs. The output of the action neural network is the sum of the setpoint command $u_{C}(t)$, the output of the feedback network $\Delta u_{B}(t)$ and the integral neural network $\Delta u_{I}(t)$. The action network is now obtained by combining the neural networks such that the output of the action neural network is equal to the sum of the outputs of the individual vector output sigmoidal feedback neural network and the vector output sigmoidal integral neural network. The input weights, output weights and the bias of action neural network can be obtained as

$$
\begin{aligned}
& W_{A}=\left[\begin{array}{cc}
W_{B} & 0 \\
0 & W_{I}
\end{array}\right] \\
& V_{A}=\left[\begin{array}{cc}
V_{B} & 0 \\
0 & V_{I}
\end{array}\right] \\
& d_{A}=\left[\begin{array}{ll}
d_{B}^{T} & d_{I}^{T}
\end{array}\right]^{T}
\end{aligned}
$$

and

$$
b_{A}=\left[\begin{array}{ll}
b_{B}^{T} & b_{I}^{T}
\end{array}\right]^{T}
$$

The action neural network obtained after the combination of the vector output sigmoidal feedback neural network and the integral neural network is as shown in Figure 18. The action neural network performance is equivalent to that of the initialized neural networks of chapter four. 


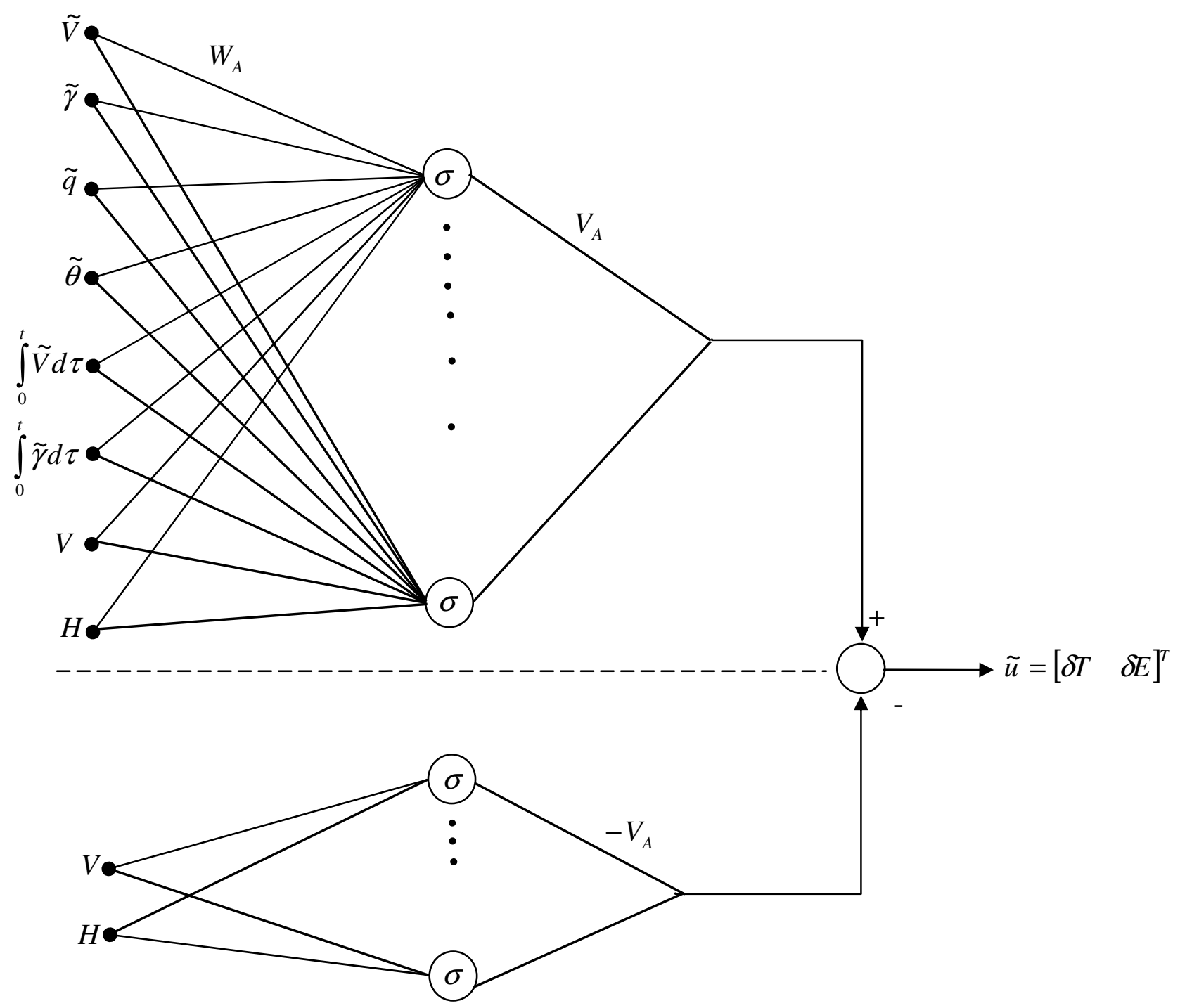

Figure 18: Action neural network.

The scalar critic neural network also needs to be converted into a vector output sigmoidal neural network. This is the only step needed to be carried out before implementing the critic neural network for the online adaptation. In chapter four the six individual neural networks were initialized separately. Now all the six individual neural networks are combined with the input weights, output weights and the biases given by 


$$
\begin{gathered}
W_{C}=\left[\begin{array}{l}
W_{C_{1}} \\
W_{C_{2}} \\
W_{C_{3}} \\
W_{C_{4}} \\
W_{C_{5}} \\
W_{C_{6}}
\end{array}\right] \\
V_{B}=\left[\begin{array}{cccccc}
V_{C_{1}} & 0 & 0 & 0 & 0 & 0 \\
0 & V_{C_{2}} & 0 & 0 & 0 & 0 \\
0 & 0 & V_{C_{3}} & 0 & 0 & 0 \\
0 & 0 & 0 & V_{C_{4}} & 0 & 0 \\
0 & 0 & 0 & 0 & V_{C_{5}} & 0 \\
0 & 0 & 0 & 0 & 0 & V_{C_{6}}
\end{array}\right] \\
d_{B}=\left[\begin{array}{llllll}
d_{C_{1}}^{T} & d_{C_{2}}^{T} & d_{C_{3}}^{T} & d_{C_{4}}^{T} & d_{C_{5}}^{T} & d_{C_{6}}^{T}
\end{array}\right]^{T}
\end{gathered}
$$

and

$$
b_{B}=\left[\begin{array}{llllll}
b_{C_{1}}^{T} & b_{C_{2}}^{T} & b_{C_{3}}^{T} & b_{C_{4}}^{T} & b_{C_{5}}^{T} & b_{C_{6}}^{T}
\end{array}\right]^{T}
$$

where the $W_{C_{n}}$ for $n=1 \ldots 6$ correspond to the input weights, $V_{C_{n}}$ for $n=1 \ldots 6$ correspond to the output weights, $d_{C_{n}}^{T}$ or $n=1 \ldots 6$ correspond to the input bias and $b_{C_{n}}^{T}$ for $n=1 \ldots 6$ correspond to the output bias of the six individual scalar networks. The combined critic neural network used for online adaptation is given in Figure 19. 


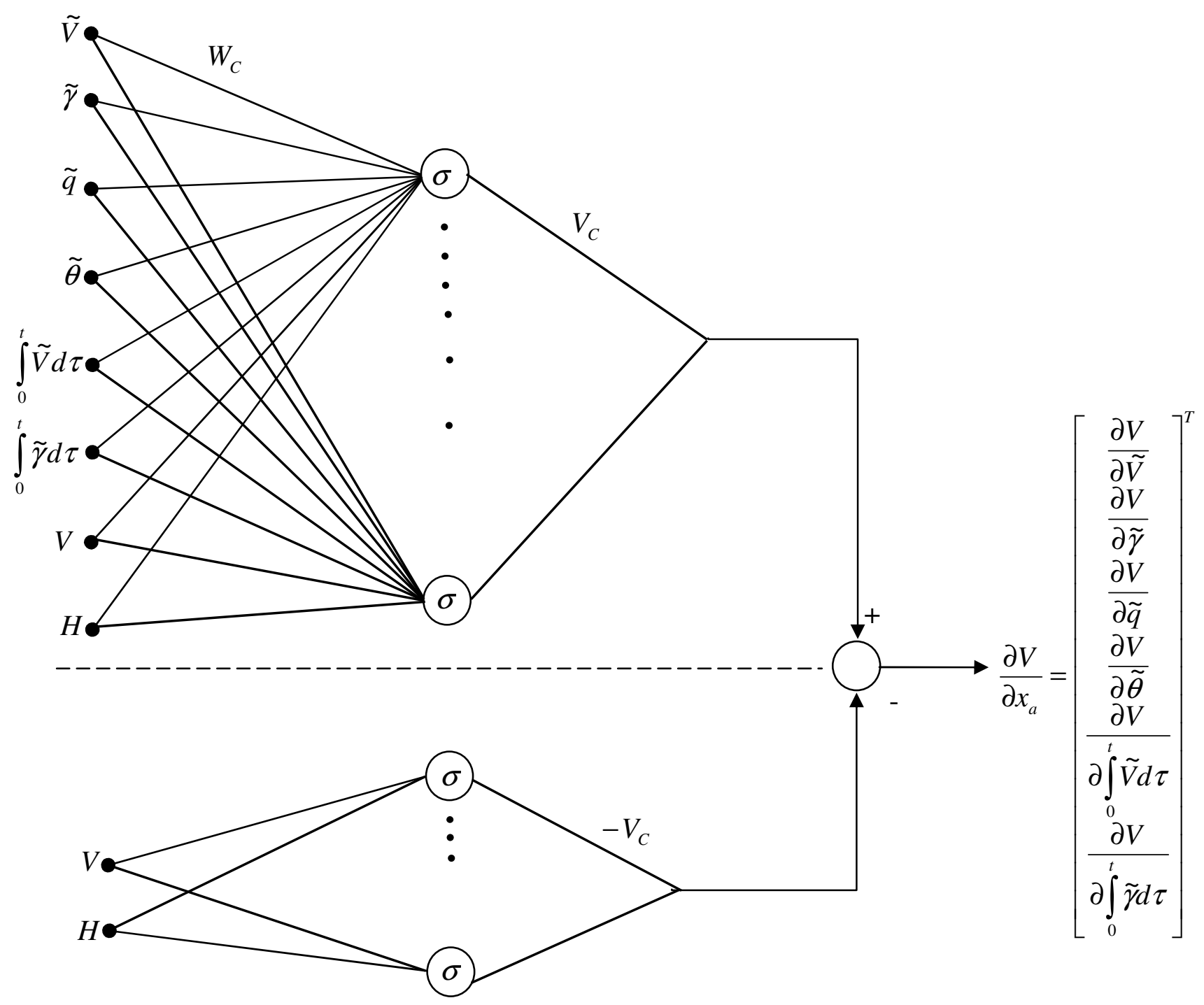

Figure 19: Critic neural network.

The action and the critic neural network that approximate the optimal control law and the gradient of the value function respectively are used in the online adaptation. The initialized action and critic neural network are adapted online to learn new system dynamics as they arise. The adaptation of the neural networks online is explained in the next section. 


\subsection{Online adaptation}

The initialized neural networks are adapted online during every time interval $\Delta t=t_{k+1}-t_{k}$ based on the actual state of the aircraft to more closely approximate the globally optimal control law. The adaptation is based on dual heuristic dynamic programming with the action network approximating the control law and the critic evaluating the gradient of the value function. This section describes the online adaptation algorithm for both the action and critic neural network.

The action neural network parameters are updated to minimize the mean squared error between the desired target, $\tilde{u}_{D}(t)$, and the actual output of the action network. The desired target for the action network is given by solving the optimality condition

$$
\frac{\partial V\left[x_{a}, t_{k}\right]}{\partial \widetilde{u}\left(t_{k}\right)}=\frac{\partial L_{D}\left[x_{a}, \tilde{u}, t_{k}\right]}{\partial \widetilde{u}\left(t_{k}\right)}+\lambda_{a}\left(t_{k+1}\right) \frac{\partial x_{a}\left(t_{k+1}\right)}{\partial \widetilde{u}\left(t_{k}\right)}=0
$$

This is a set of nonlinear equations which is solved numerically using the MATLAB function fsolve. The initial guess, $\tilde{u}_{D}\left(t_{k}\right)^{G}$, used in the solution of these equations is the output of the action network with the weights from the previous time step. The algorithm for updating the action network is shown in Figure 20. The derivative of the Lagrangian is found analytically given by

$$
\frac{\partial L_{S D}\left[x_{a}, \tilde{u}, t_{k}\right]}{\partial \widetilde{u}\left(t_{k}\right)}=x_{a}^{T}\left(t_{k}\right) M_{a}+\tilde{u}^{T}\left(t_{k}\right) R_{a}
$$

The discrete equivalent of the system model given by

$$
x_{a}\left(t_{k+1}\right)=f_{a D}\left[x_{a}, \tilde{u}, t_{k}\right]
$$

is used to determine the transition matrix $\frac{\partial x_{a}\left(t_{k+1}\right)}{\partial \tilde{u}\left(t_{k}\right)}$. The system model is also necessary to find the output of the critic network $\lambda_{a}\left(t_{k+1}\right)$ with the weights of the critic network being equal to that 
during the previous time step. Once the optimality condition is solved to obtain the target for the action network i.e. $\tilde{u}_{D}\left(t_{k}\right)$, the weights of the action network are now adapted using the modified resilient back propagation algorithm.

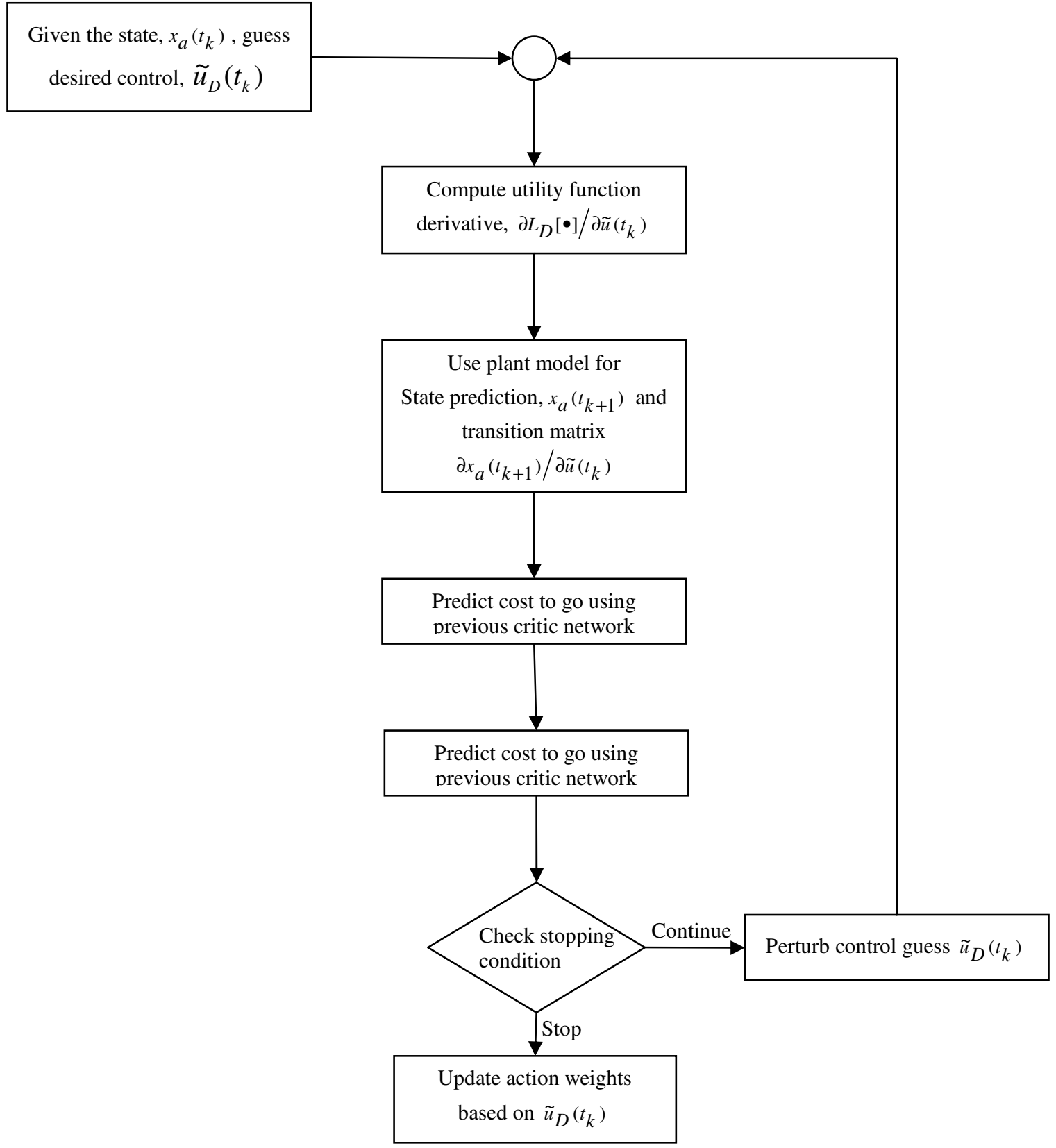

Figure 20: Action network adaptation. 
Once the action network is updated, the desired output of the critic $\lambda_{a D}\left(t_{k}\right)$ is computed based on the current state of the system $x_{a}\left(t_{k}\right)$. The critic updating algorithm is as shown in Figure 21. The output of the critic network is given by

$$
\lambda_{a D}\left(t_{k}\right) \equiv \frac{\left.\partial \bigvee x_{a}, t_{k}\right]}{\partial x_{a}\left(t_{k}\right)}=\frac{\partial L_{D}\left[x_{a}, \tilde{u}, t_{k}\right]}{\partial x_{a}\left(t_{k}\right)}+\frac{\partial L_{D}\left[x_{a}, \tilde{u}, t_{k}\right]}{\partial \tilde{u}\left(t_{k}\right)} \frac{\partial \tilde{u}\left[x\left(t_{k}\right)\right]}{\partial x_{a}\left(t_{k}\right)}+\lambda_{a}\left(t_{k+1}\right) \frac{\partial x_{a}\left(t_{k+1}\right)}{\partial x_{a}\left(t_{k}\right)}+\lambda_{a}\left(t_{k+1}\right) \frac{\partial x_{a}\left(t_{k+1}\right)}{\partial \tilde{u}\left(t_{k}\right)} \frac{\partial \tilde{u}\left[x\left(t_{k}\right)\right]}{\partial x_{a}\left(t_{k}\right)}
$$

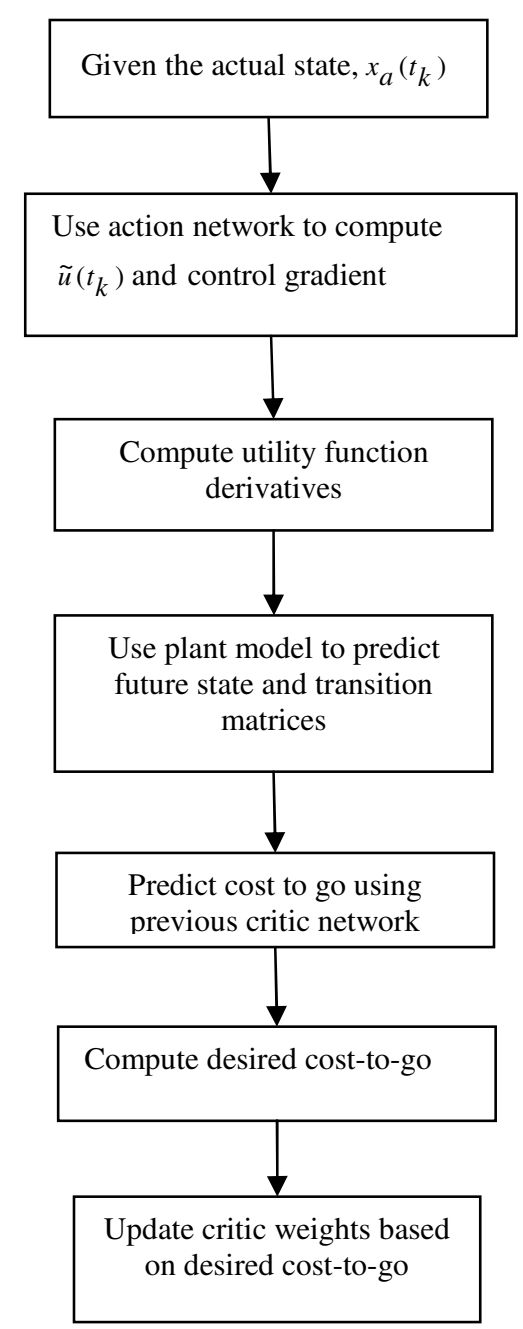

Figure 21: Critic network adaptation.

The derivative of the Lagrangian with respect to the state is obtained numerically using 


$$
\frac{\partial L_{D}\left[x_{a}, \tilde{u}, t_{k}\right]}{\partial x_{a}\left(t_{k}\right)}=x_{a}^{T}\left(t_{k}\right) Q_{a}+\left[M_{a} \tilde{u}\left(t_{k}\right)\right]^{T}
$$

During the adaptation of the critic the actual value of the control $\tilde{u}\left(t_{k}\right)$ is known, hence the control gradient can be calculated. In addition the discretized form of the system equation can be used to obtain the transition matrices $\frac{\partial x_{a}\left(t_{k+1}\right)}{\partial \tilde{u}\left(t_{k}\right)}$ and $\frac{\partial x_{a}\left(t_{k+1}\right)}{\partial x_{a}\left(t_{k}\right)}$. Now the critic network with the parameters from the previous time step is used to calculate $\lambda_{a}\left(t_{k+1}\right)$ based on the predicted value of $x_{a}\left(t_{k+1}\right)$ obtained from the model of the plant. Once the desired output of the critic network is obtained, $\lambda_{a D}\left(t_{k}\right)$, the parameters of the critic neural networks are updated using the modified resilient back propagation algorithm.

The updating of the action and critic network is done for every time step $\Delta t$. Since the updating of the action and critic network is obtained from the recurrence relation of dynamic programming, it is seen that the system converges to the optimal trajectory over time. It is also observed that the adaptation of the networks allows the nonlinear controller to meet performance specifications in the presence of nonlinearities as well as multiple control failures. 


\section{SIMULATION AND RESULTS}

The adaptive neural network controller is used to control the three degree of freedom simulation of the general aviation aircraft. The simulation is carried out for different command inputs of velocity and flight path angle. The results of the simulation are observed for parameter variations and control failures. The online adaptation of the neural network controller prevents loss of stability of the aircraft and tracks pilot input commands.

\subsection{Simulation}

The adaptive neural controller is evaluated using the simulation of a three degree of freedom general aviation aircraft. The motion of the aircraft in the longitudinal plane is governed by the following three nonlinear differential equations [26]. The three equations constitute the force along the $\mathrm{x}$-axis, $\mathrm{z}$-axis and moment about the $\mathrm{y}$-axis. One more equation called the kinematic equation which relates the pitch rate $Q$ with the pitch angle

$$
\begin{gathered}
m(\dot{U}+Q W)=-m g \sin \theta+F_{A_{x}}+F_{T_{x}} \\
m(\dot{W}-Q U)=+m g \cos \theta+F_{A_{z}}+F_{T_{z}} \\
I_{y y} \dot{Q}=M_{A}+M_{T} \\
Q=\dot{\theta}
\end{gathered}
$$

The aerodynamic forces and moments in equations (143), (144) and (145) can be written in terms of the stability derivatives. The dynamics of the aircraft are characterized by these stability derivatives. The aerodynamic forces and moments in terms of the stability derivatives are given by equations (146), (147) and (148).

$$
F_{A_{x}}=-\left(C_{D_{0}}+C_{D_{k}} * C_{L}^{2}\right) q S
$$




$$
\begin{aligned}
& F_{A_{Z}}=q S\left(C_{Z_{0}}+C_{Z_{\alpha}} \alpha+C_{Z_{\dot{\alpha}}} \hat{\dot{\alpha}}+C_{Z_{q}} \hat{Q}+C_{Z_{\delta_{e}}} \delta_{e}\right) \\
& M_{A}=q S \bar{c}\left(C_{m_{0}}+C_{m_{\alpha}} \alpha+C_{m_{\dot{\alpha}}} \hat{\dot{\alpha}}+C_{m_{q}} \hat{Q}+C_{m_{\delta_{e}}} \delta_{e}\right)
\end{aligned}
$$

where $q$ is the dynamic pressure, $S$ is the reference area of the wing and $\bar{c}$ is the chord length.

The aircraft is then simulated by integrating the non linear equations of motion in the MATLAB environment numerically. The equations of motion are integrated using the fourth order rungekutta method with a sampling time of 0.05 seconds. This simulation is used as the actual aircraft in the evaluation of the adaptive critic neural network controller. The controller is evaluated for unanticipated control failures as well as for parameter variations. The adaptive critic algorithm is also evaluated in the presence of nonlinearity at velocities close to the stall velocity. The code used for simulations of the adaptive critic algorithm had a limitation in that it only accepted step input commands as well as modeling errors could only be simulated at time $t=0$. Also for all the cases other than the case near stall, the altitude of the aircraft is not shown because the altitude remains constant.

\subsection{Results}

Case 1: Adaptive control during partial elevator failure

The capability of the neural network based adaptive control system to adapt to partial elevator failure is considered first. The aircraft is initially flying at $\left(V_{0}, h_{0}\right)=\left(181.23 \mathrm{fts}^{-1}, 3000 \mathrm{ft}\right)$, which is an interpolation point. A step input of two degrees in flight path angle is commanded at time $t=0$. A partial elevator failure is simulated by changing the parameter $C_{m_{\delta_{E}}}$ and $C_{L_{\delta_{E}}}$ by $10 \%$ at time $t=0$. The response of the neural network controller is first evaluated when the neural networks are not adapted online i.e the PINN controller. The response of the initialized neural network without adaptation is shown in Figures 22 and 23. 
Figure 22 is a plot of velocity $V$, with respect to time, $t$, and Figure 23 is a plot of throttle setting, $\delta_{T} \%$, versus time $t$. As shown in Figures 22 and 23, the dynamic response of the aircraft with PINN controller deteriorates in the presence of partial elevator failure as can be seen from the slightly increased overshoot but with an increased settling time. The aircraft retains stability even in the presence of partial elevator failure since LQR theory is used in the design of the initialized controller resulting in a robust control design. In the next step, the same simulation is carried out but the neural networks are allowed to adapt online as explained in the previous section. The response of the neural network controller with adaptation is also shown in the same Figures 22 and 23 with a solid line. As observed from the response we see that the overshoot in flight path angle is reduced and the settling time is increased as compared to the PINN controller. The action and critic neural network weights are adapted to the changed dynamics of the aircraft due to partial failure in the elevator. The incremental cost $J$ is also shown in figure 25, As observed from the plots the incremental cost associated with the adaptive critic is less than that of the PINN controller during the transient phase of the response. As the response reaches steady state value we see a ramp in the incremental cost associated with the adaptive critic, this is due to the steady state error associated with the adaptive critic designs.
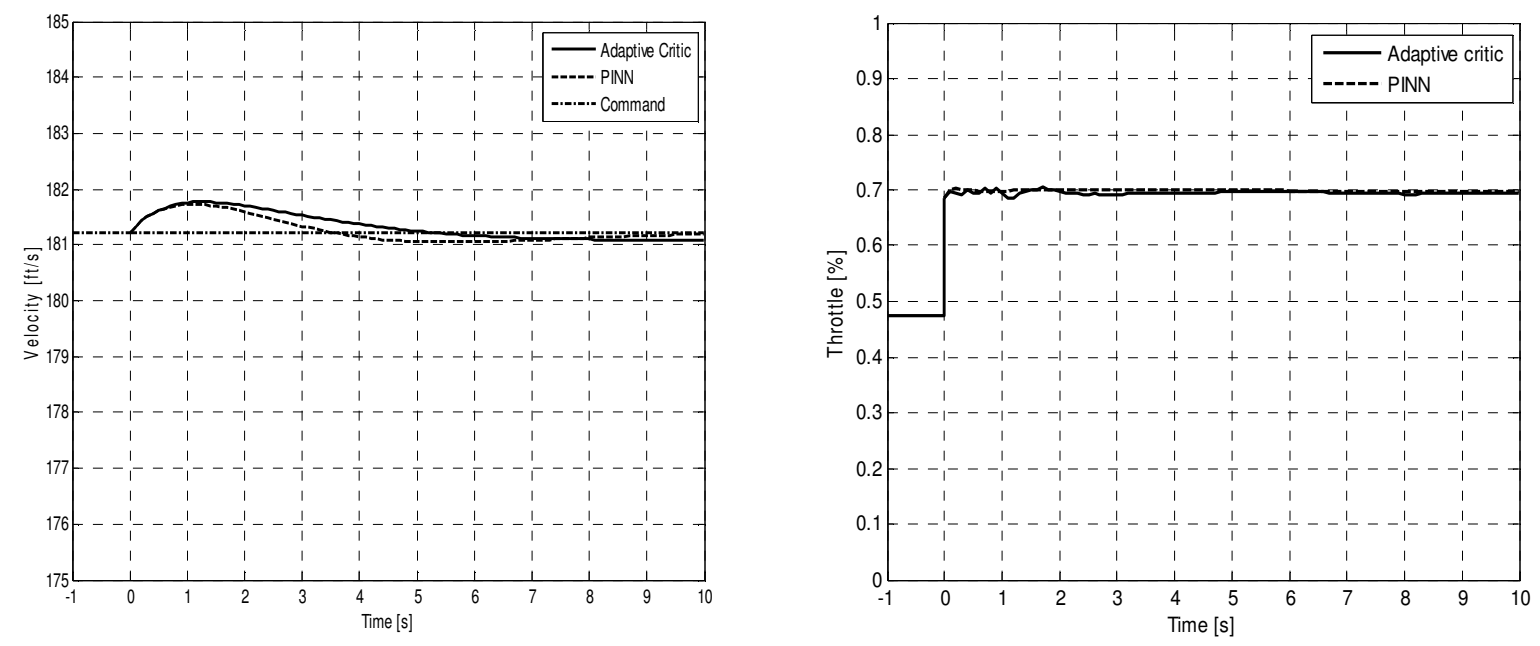
Figure 22: Velocity response and throttle setting for partial elevator failure.
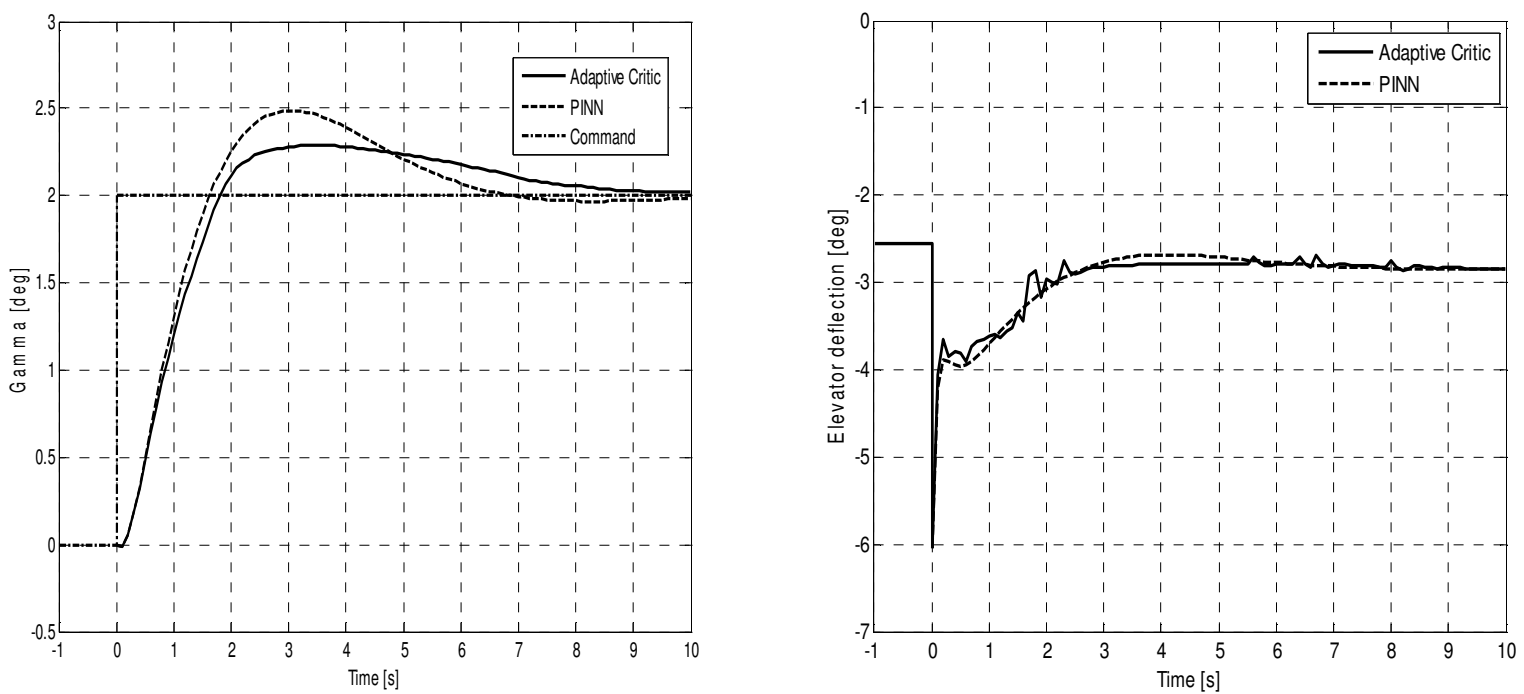

Figure 23: Flight path angle response and elevator deflection for partial elevator failure.
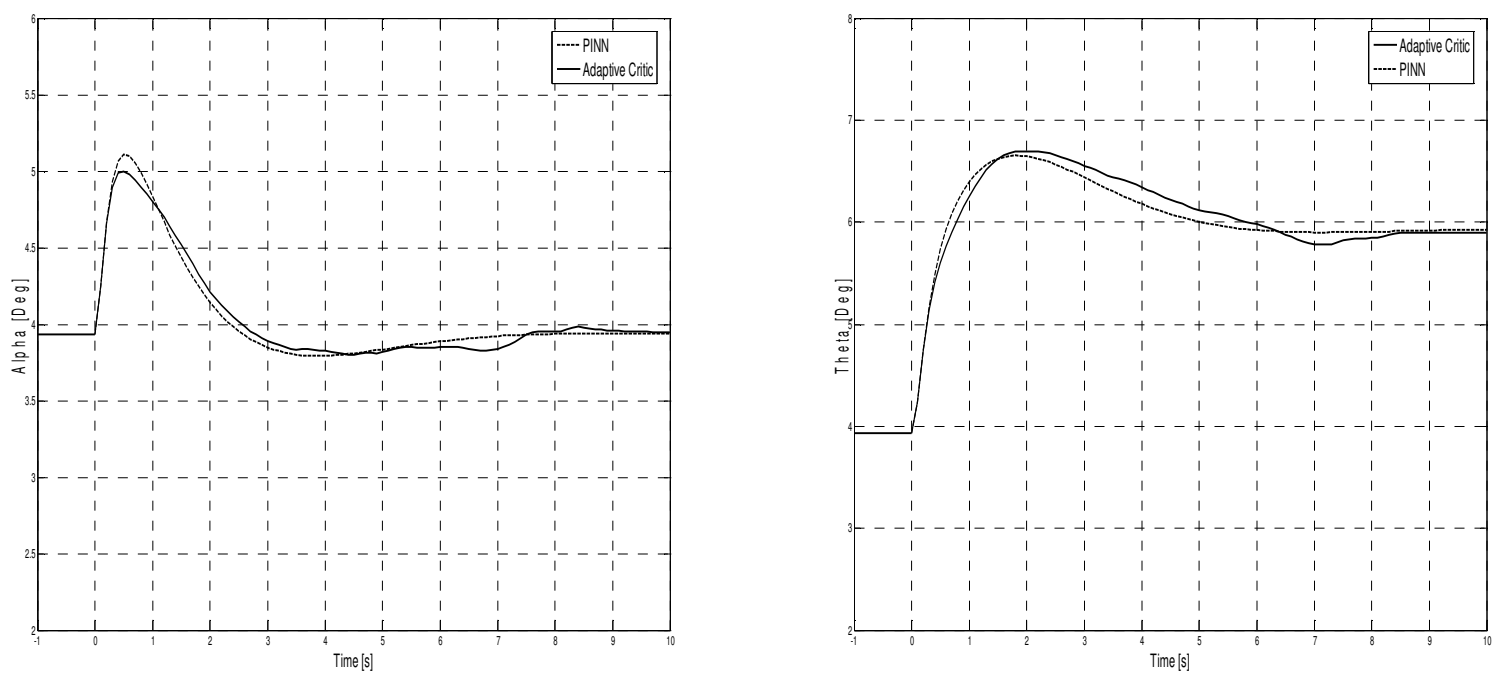

Figure 24: Angle of attack and pitch angle for partial elevator failure. 


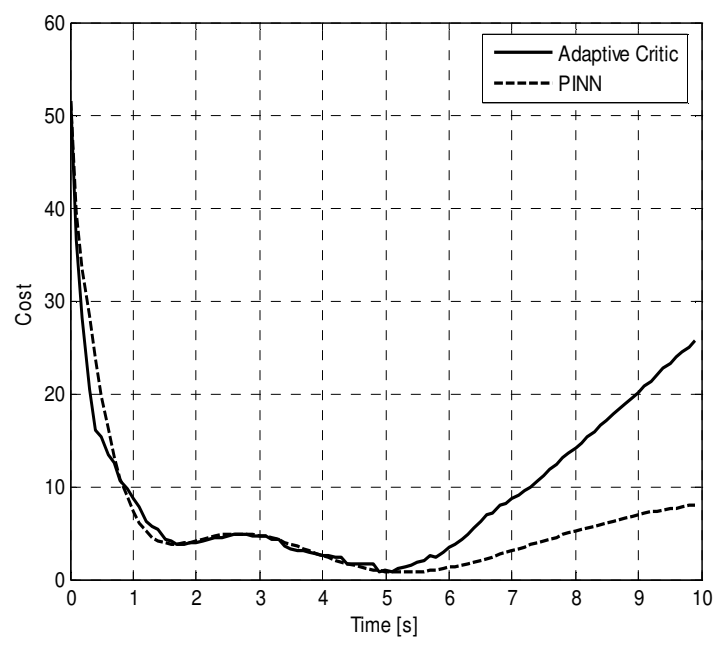

Figure 25: Incremental cost for partial elevator failure.

Case 2: Adaptive control during partial thrust failure

The capability of the neural network based adaptive control system to a sudden loss of thrust is evaluated. As in the previous simulation, the aircraft is initially flying at $\left(V_{0}, h_{0}\right)=\left(181.23 \mathrm{fts}^{-1}, 3000 \mathrm{ft}\right)$. A step increase of five feet per second in velocity is introduced to the system. A partial throttle gearing failure is simulated by reducing the available thrust by $10 \%$. The response of the neural network controller is then evaluated when the neural networks are not adapted online. The response of the initialized neural network without adaptation is as shown as a dotted line in Figures 26 and 27. As observed from the plots, the aircraft takes longer to settle at the pilot input command for velocity and also results in a slightly larger overshoot. The same simulation is now carried out but the neural networks are allowed to adapt online. The response of the neural network controller with online adaptation is also shown as a solid line in Figures 26 and 27. As observed from the response the overshoot is slightly reduced as compared to that of the initialized neural network controller in the presence of partial throttle gearing failure but it also results in a slightly smaller settling time as compared to the initialized controller. . The incremental cost $J$ is also shown in figure 29, As observed from the plots the 
incremental cost associated with the adaptive critic is less than that of the PINN controller during the transient phase of the response. As the response reaches steady state value we see a ramp in the incremental cost associated with the adaptive critic, this is due to the steady state error associated with the adaptive critic designs
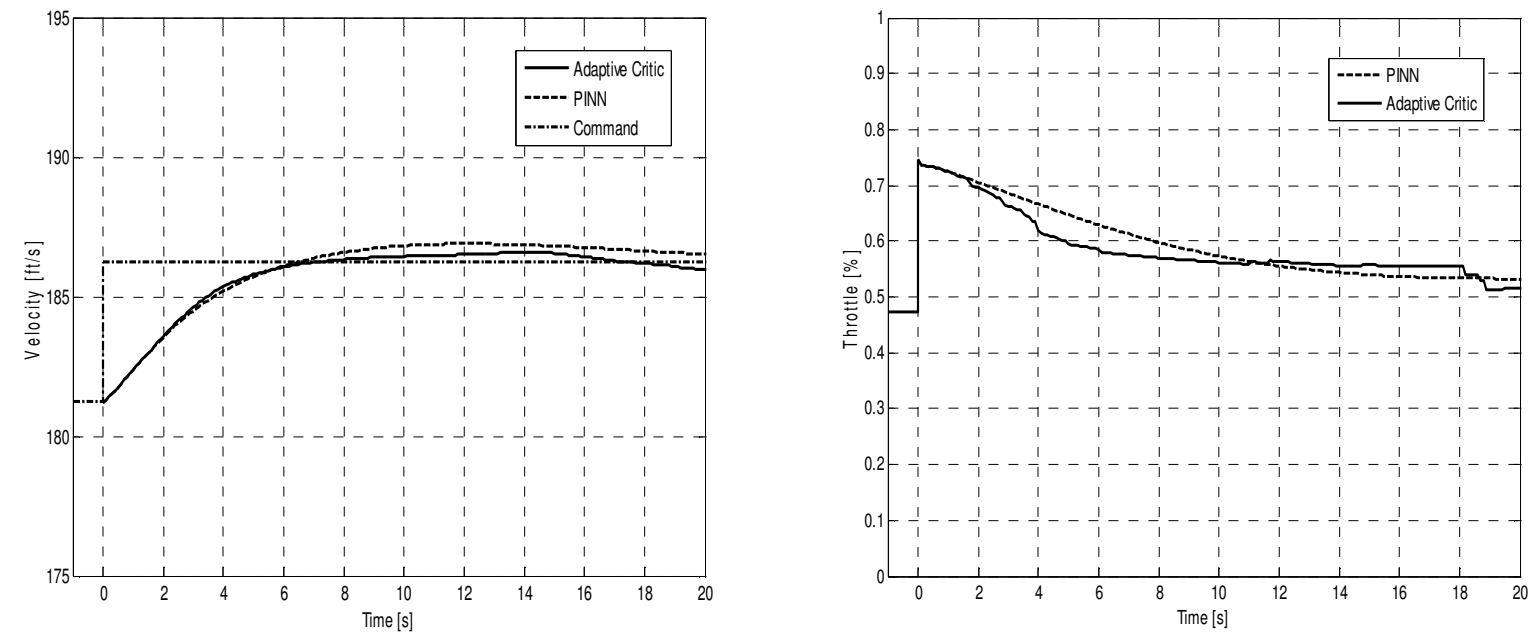

Figure 26: Velocity response and throttle setting for partial thrust failure.
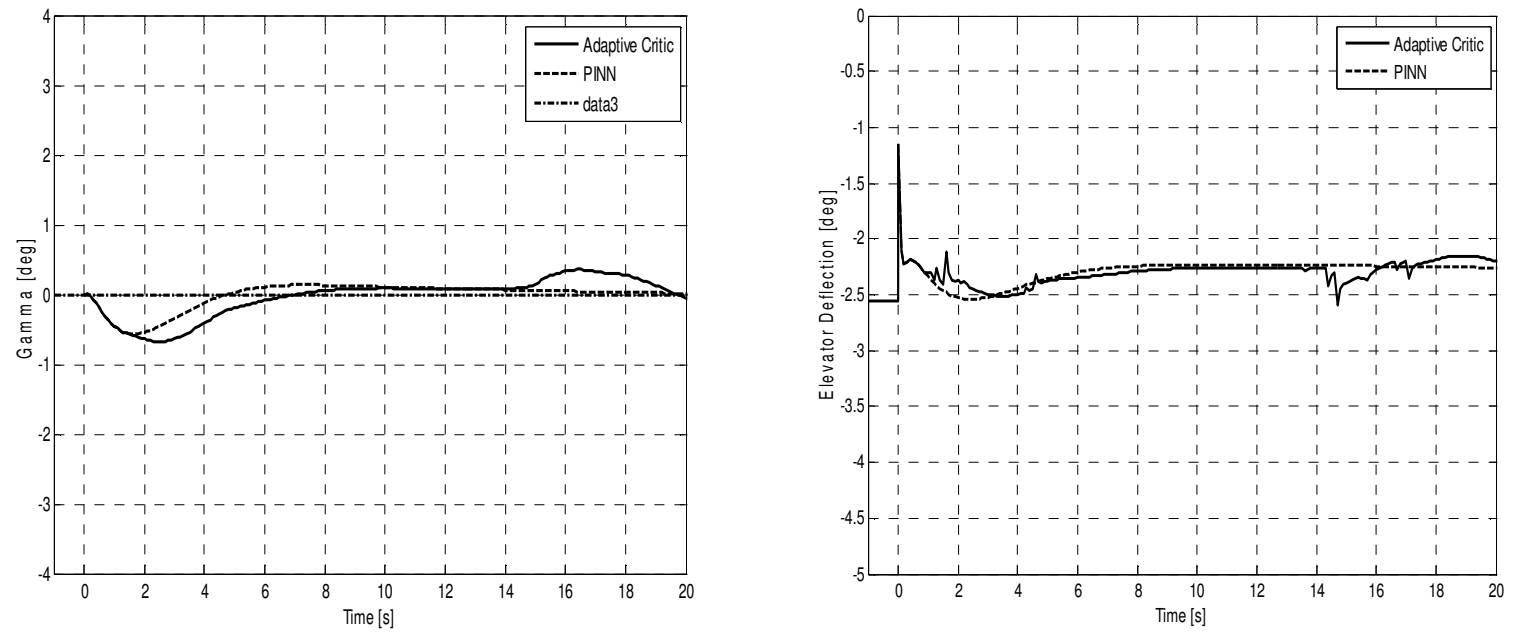

Figure 27: Flight path angle response and elevator deflection for partial thrust failure. 

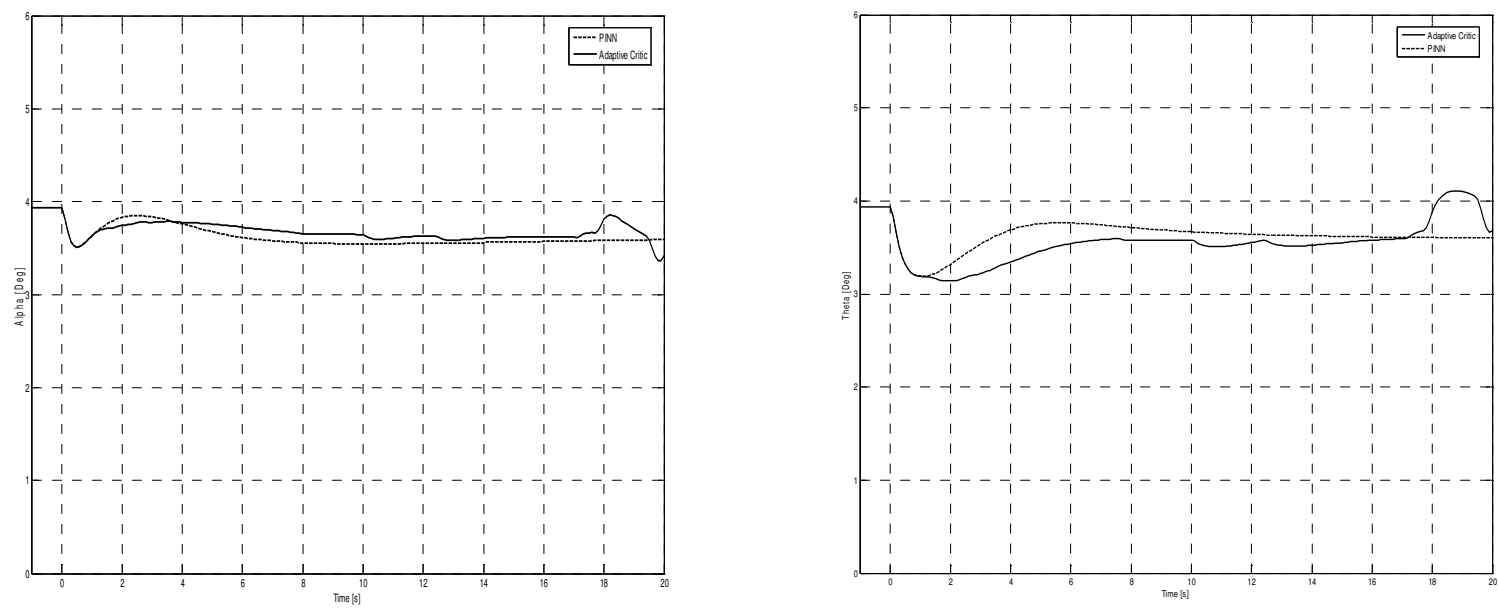

Figure 28: Angle of attack and pitch angle for partial thrust failure.

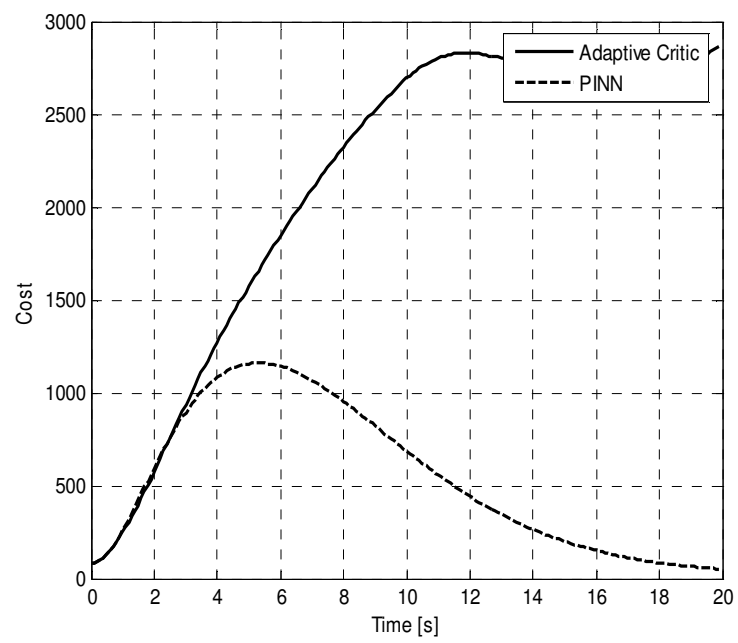

Figure 29: Incremental cost for partial thrust failure.

Case 3: Adaptive control during parameter variations

The capability of the neural network based adaptive control system is now evaluated for modeling error between the assumed and the actual model. The aircraft is initially flying at $\left(V_{0}, h_{0}\right)=\left(181.23 \mathrm{fts}^{-1}, 3000 \mathrm{ft}\right)$. A step input of two degrees in flight path angle is commanded at $\mathrm{t}=0$. The parameters $C_{m_{\alpha}}$ and $C_{m_{q}}$ are changed by $10 \%$ to simulate modeling error between the aircraft and the assumed model. The response of the initialized neural network controller is evaluated in this scenario. The response of the initialized neural network without adaptation is as 
shown with a dotted line in Figures 30 and 31. As observed from the response of the initialized controller the overshoot in the response of the flight path angle increases as compared to the response with the ideal model. The same simulation is now carried out but the neural networks are allowed to adapt online. The response of the neural network controller that is adapted online is also shown in Figures 30 and 31 as a solid line. As observed from the response we see that the overshoot in the flight path angle is smaller as compared to that of the initialized controller in the presence of the modeling error. However we see an increase in settling time as compared to the initialized controller. The incremental cost $J$ is also shown in figure 33, As befor the incremental cost associated with the adaptive critic is less than that of the PINN controller during the transient phase of the response. As the response reaches steady state value we see a ramp in the incremental cost associated with the adaptive critic, this is due to the steady state error associated with the adaptive critic designs
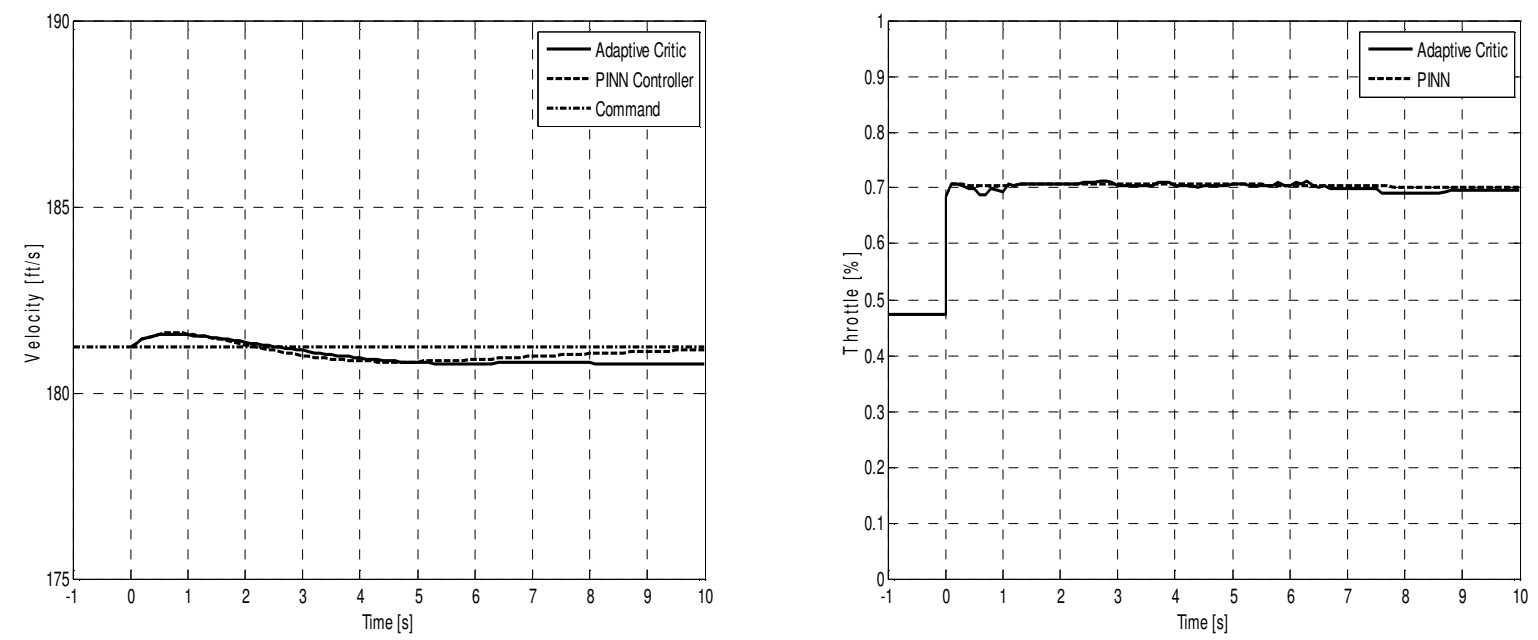

Figure 30: Velocity response and throttle setting for variation in $C_{m_{\alpha}}$ and $C_{m_{q}}$. 

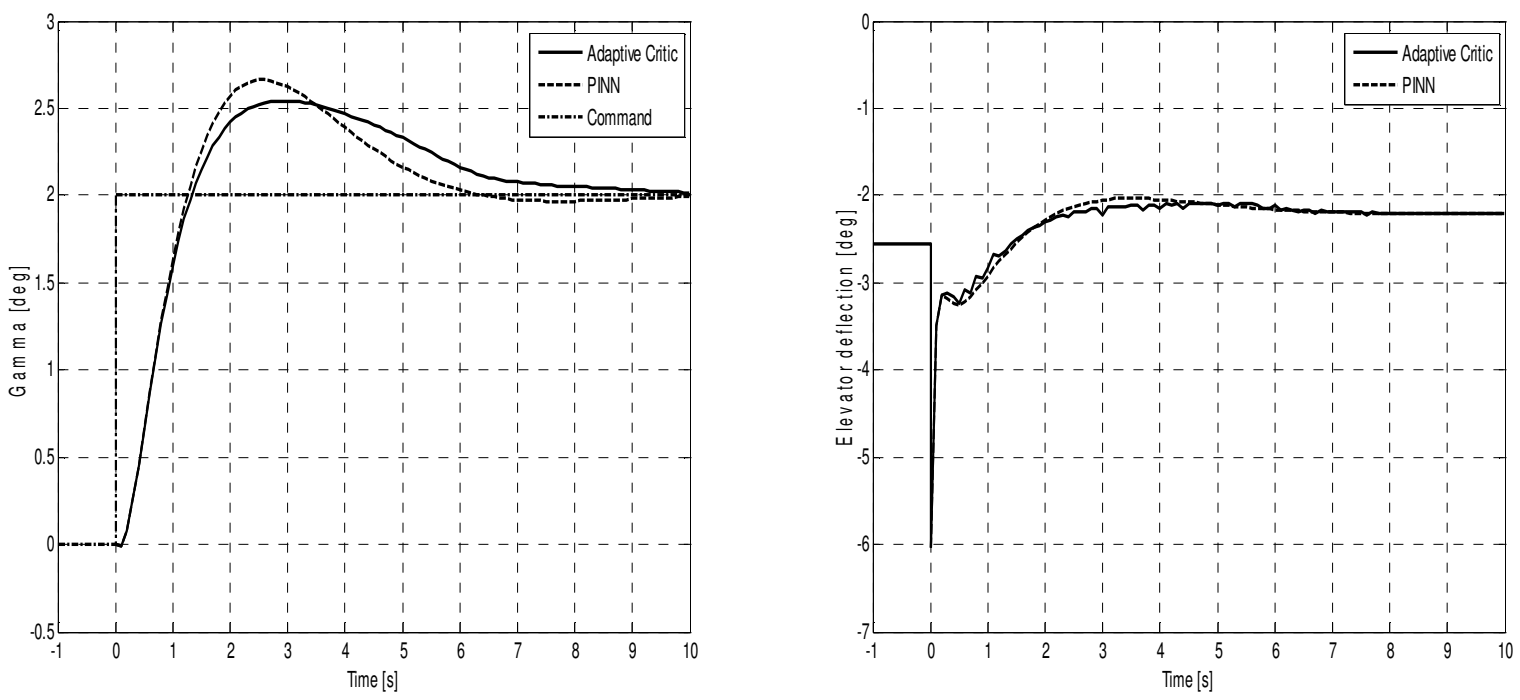

Figure 31: Flight path angle response and elevator deflection for variation in $C_{m_{\alpha}}$ and $C_{m_{q}}$.
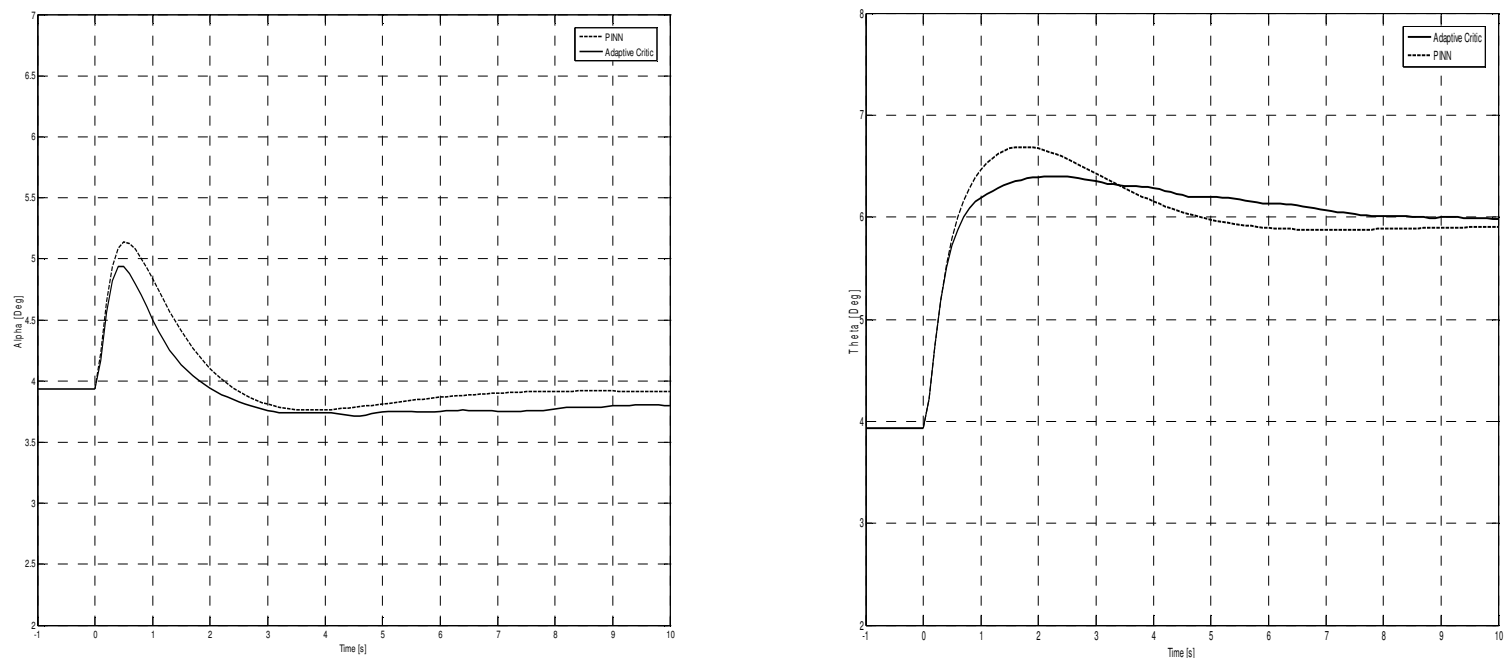

Figure 32: Angle of attack and pitch angle for variation in $C_{m_{\alpha}}$ and $C_{m_{q}}$. 


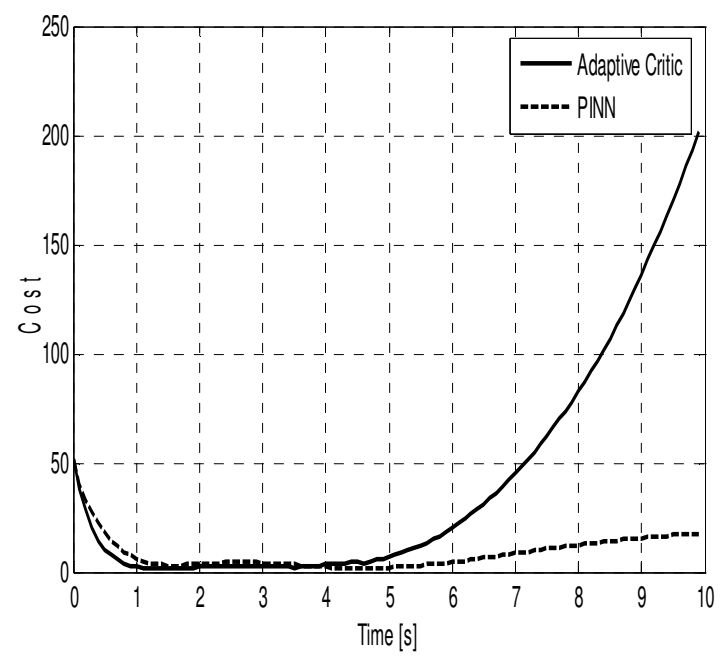

Figure 33: Incremental cost for variation in $C_{m_{\alpha}}$ and $C_{m_{q}}$.

Next the neural network based adaptive control system is evaluated for a different modeling error between the assumed and the actual model. The aircraft is initially flying at $\left(V_{0}, h_{0}\right)=\left(181.23 \mathrm{fts}^{-1}, 3000 \mathrm{ft}\right)$. A step increase of five feet per second in velocity is commanded. The parameter $C_{D_{k}}$ is decreased by $10 \%$. The response of the initialized neural network controller is evaluated in this scenario. The response of the initialized neural network without adaptation is as shown in Figures 34 and 35. As observed from the plots the aircraft response has deteriorated in the presence of modeling error between the actual aircraft and the assumed model. The same simulation is now carried out but the neural networks are allowed to adapt online. The response of the neural network controller that is adapted online is also shown in Figure 34 and Figure 35. As observed from the response the online adaptation of the neural network reduces overshoot but results in a slightly larger settling time. . The incremental cost $J$ is also shown in figure 37, The incremental cost associated with the adaptive critic is less than that of the PINN controller during the transient phase of the response. As the response reaches 
steady state value we see a ramp in the incremental cost associated with the adaptive critic, this is due to the steady state error associated with the adaptive critic designs
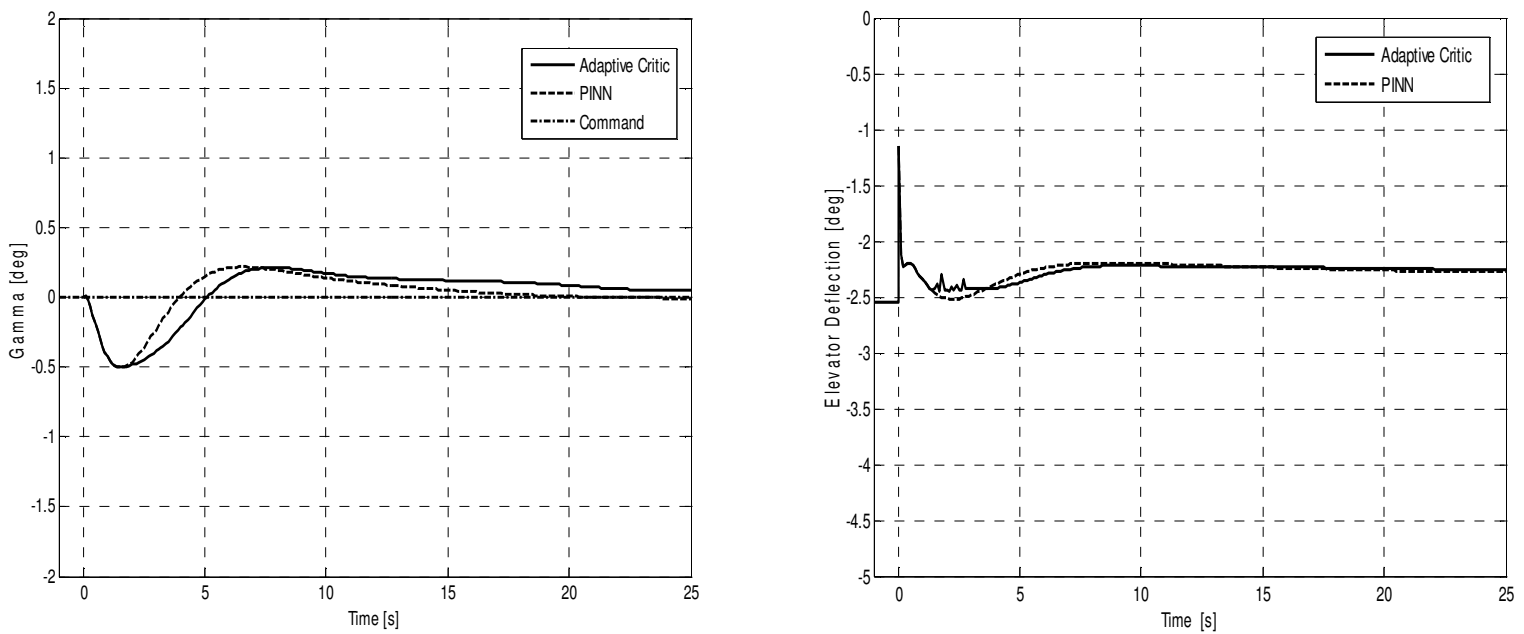

Figure 34: Flight path angle response and elevator deflection for variation in $C_{D_{k}}$.
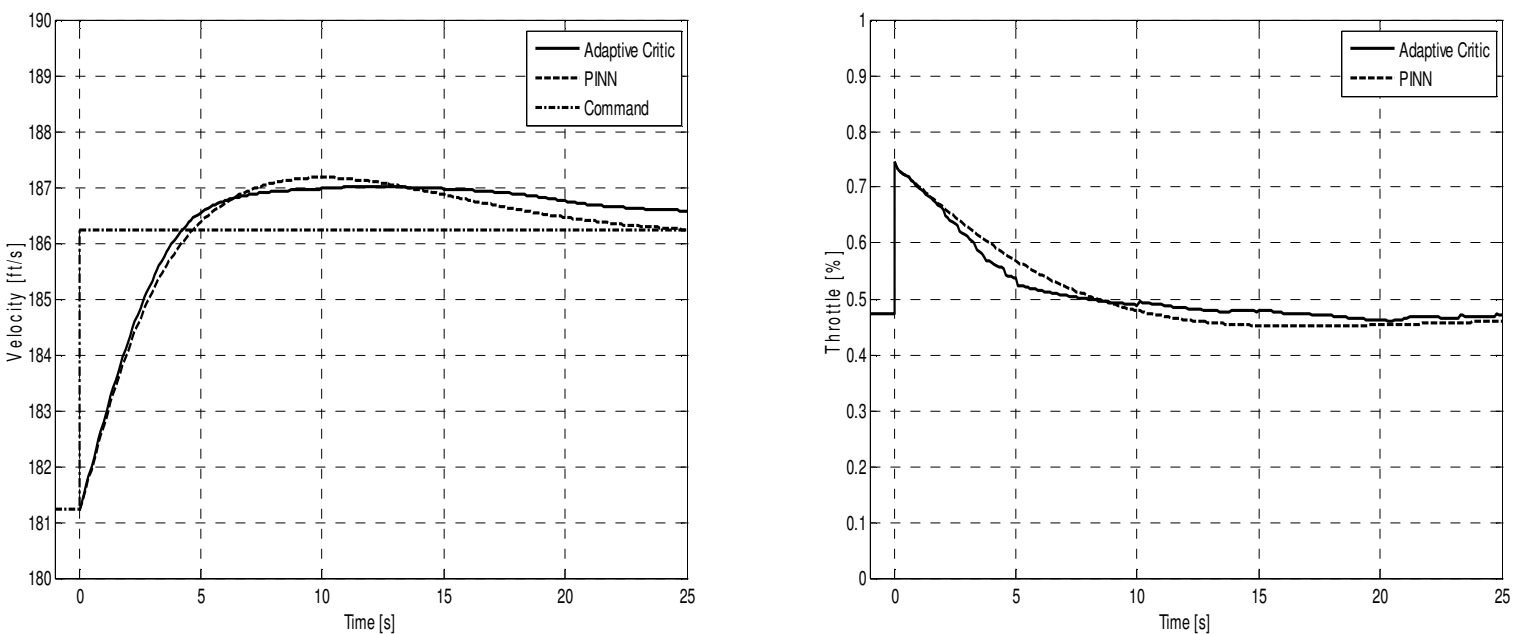

Figure 35: Velocity response and throttle setting for variation in $C_{D_{k}}$. 

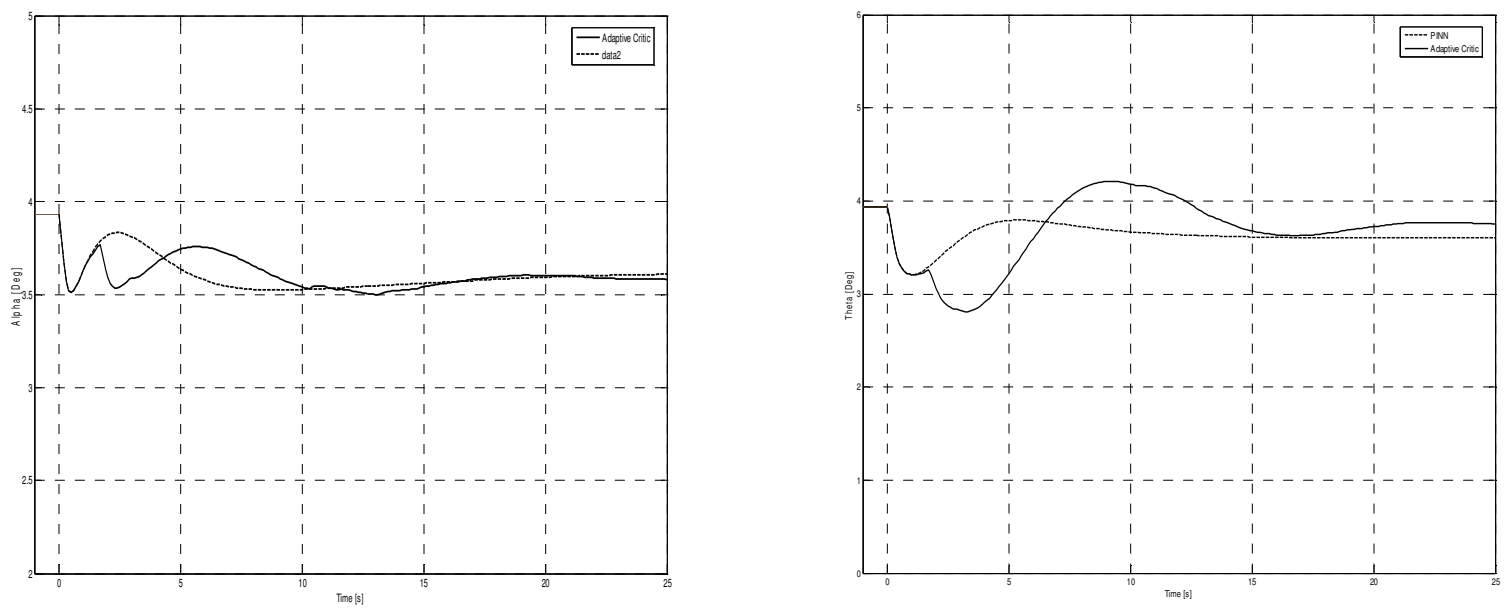

Figure 36: Angle of attack and pitch angle for variation in $C_{D_{k}}$.

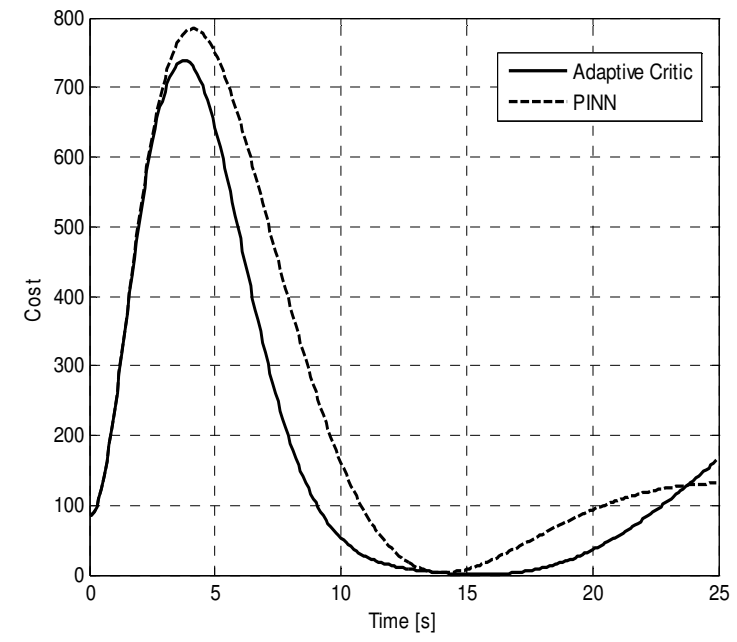

Figure 37: Incremental cost for variation in $C_{D_{k}}$.

Case 4: Adaptive control near stall

The capability of the neural network based adaptive control system is now evaluated for large inputs close to the stall. The aerodynamic model of the Bonanza was modified to include the stall model in the lift curve. The stall model incorporated is different than the actual stall of the Bonanza, with the stall velocity much greater than the stall velocity of the actual Bonanza. In the stall model used in this thesis, the stall angle of attack $\alpha_{\text {stall }}$ was set to nine degrees, which 
resulted in a stall velocity of $135 \mathrm{fts}^{-1}$ (80 Knots). Initially, the aircraft is flying at $\left(V_{0}, h_{0}\right)=\left(181.23 \mathrm{fts}^{-1}, 3000 \mathrm{ft}\right)$. A step input of minus forty feet per second in velocity is commanded at $\mathrm{t}=0$. The response of the initialized neural network controller is evaluated in this scenario. The response of the initialized neural network without adaptation is as shown with a dotted line in Figures 38 and 39. As observed from the response of the initialized controller the aircraft stalls and is unable to maintain the commanded airspeed. The same simulation is now carried out but the neural networks are allowed to adapt online. The response of the neural network controller that is adapted online is also shown in Figures 38 and 39 as a solid line. As observed from the response we see that the adaptation prevents the aircraft from becoming unstable and tracks pilot input command. The initialized PINN controller fails in this scenario due the non-linearity of the drag and lift becoming significant at low speeds near stall which is far from the initial trim velocity. As seen from the plot of incremental cost over time, we see that the cost associated with the adaptive critic controller is less than the PINN controller.
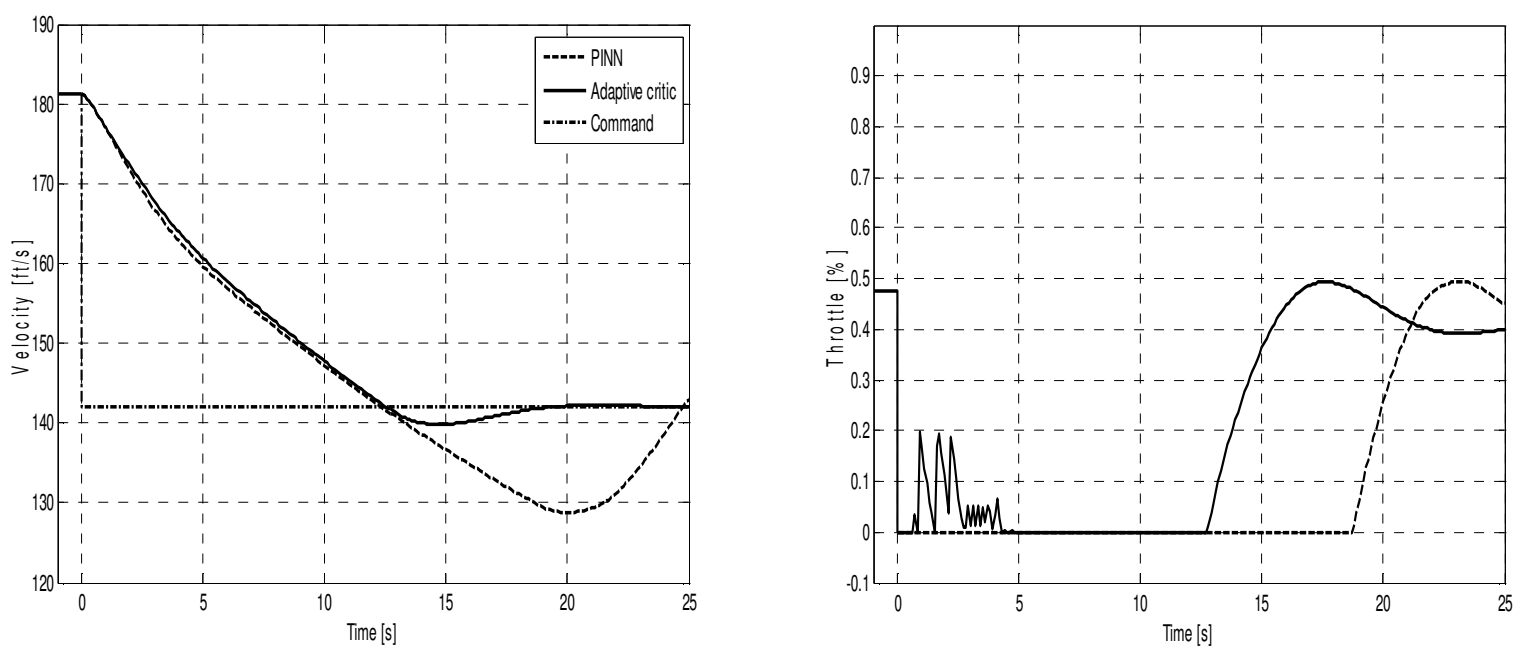

Figure 38: Velocity response and throttle setting for a command of $-40 \mathrm{ft} / \mathrm{s}$ in velocity. 

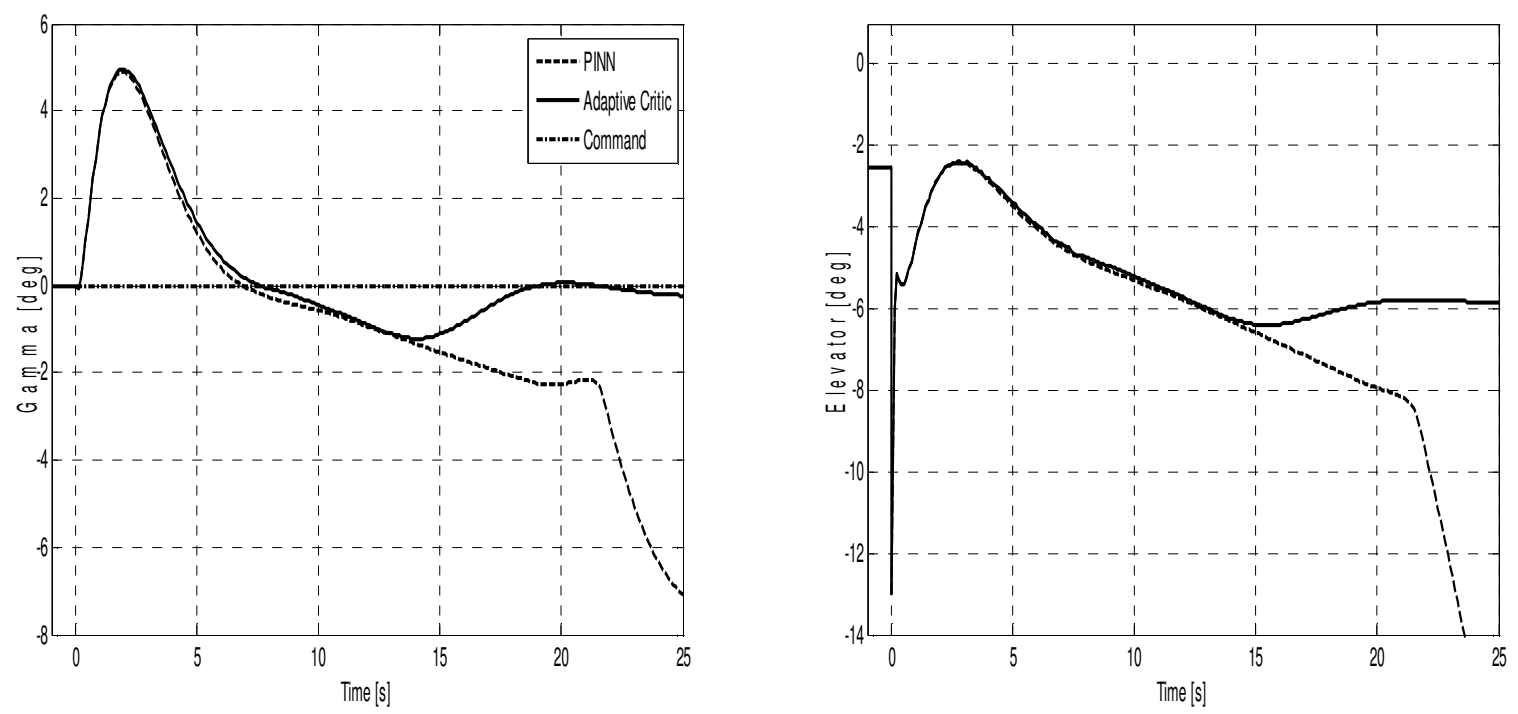

Figure 39: Flight path angle response and elevator deflection for a command of $-40 \mathrm{ft} / \mathrm{s}$ in velocity.
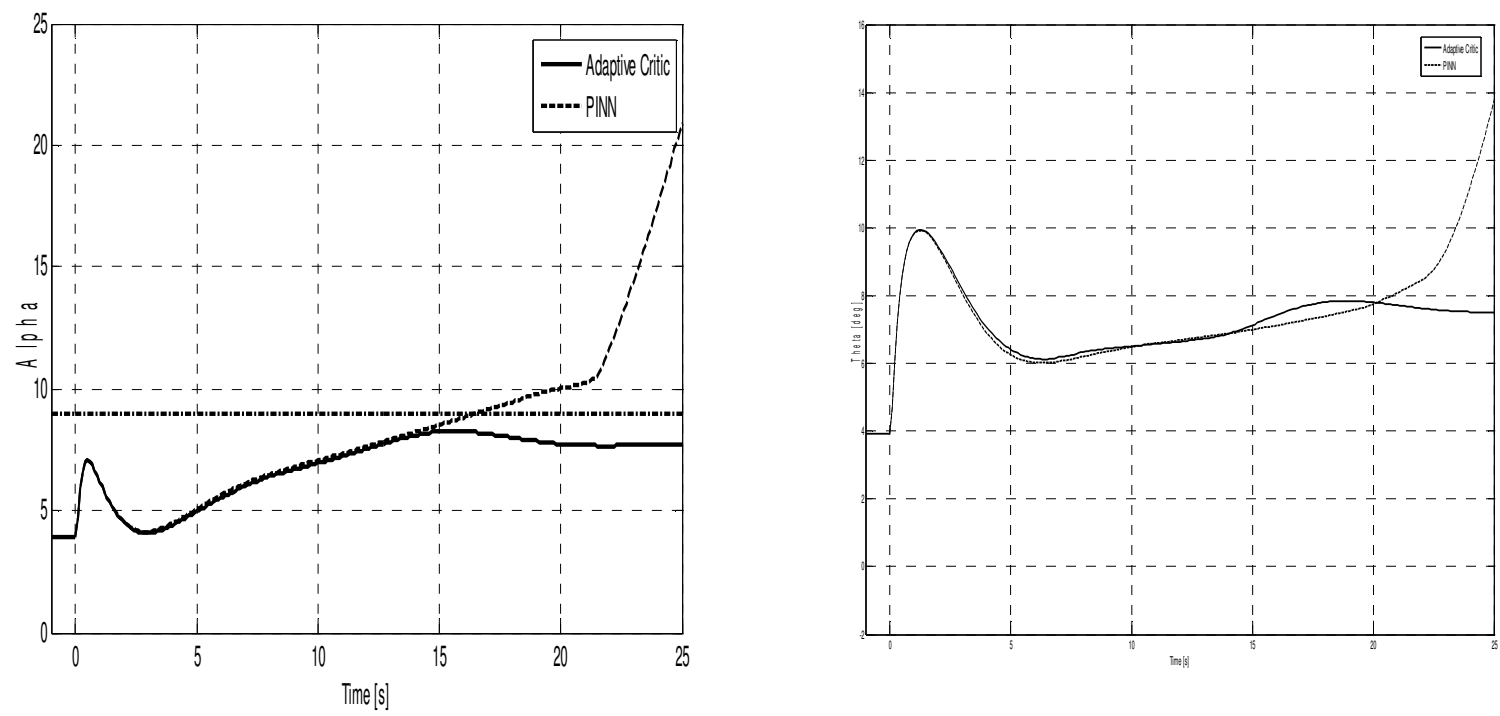

Figure 40: Angle of attack and theta time history for a command of $-40 \mathrm{ft} / \mathrm{s}$ in velocity. 


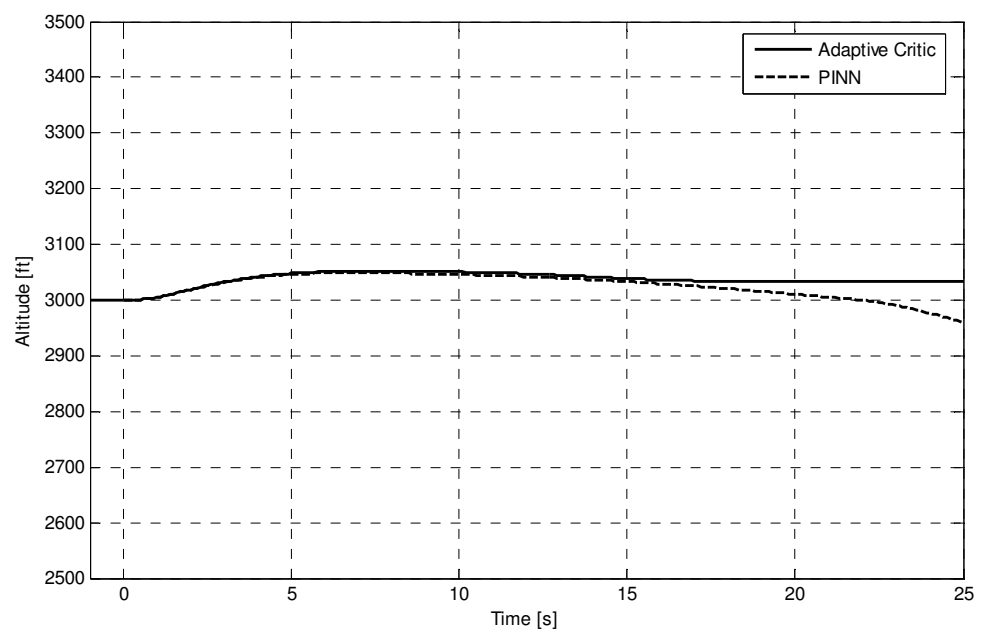

Figure 41: Altitude time history for a command of $-40 \mathrm{ft} / \mathrm{s}$ in velocity.

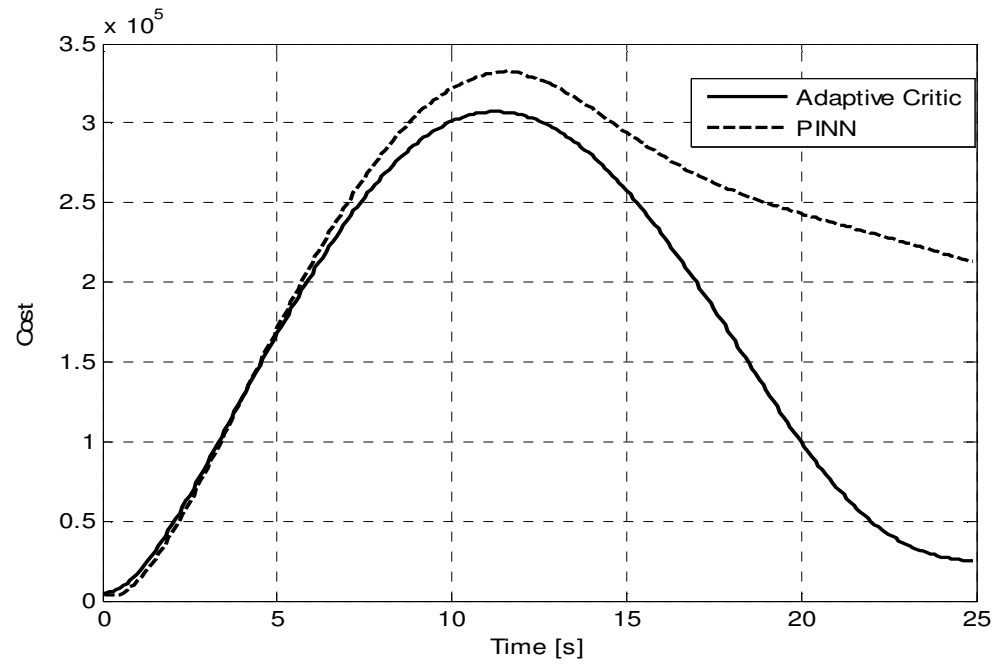

Figure 42: Incremental cost for a command of $-40 \mathrm{ft} / \mathrm{s}$ in velocity.

In this chapter the performance of the adaptive critic based controller is evaluated using simulations of the Bonanza. From the results it is seen that the adaptive critic controller results in a slight reduction in overshoot as compared to the initialized controller in the presence of unanticipated control failures and modeling errors. From the plots for modeling errors it is observed that the PINN controller works well in the linear region of the flight envelope. In order to test the PINN controller in the nonlinear region of the flight envelope a case near stall velocity of the aircraft is considered. For the case near stall, it is seen that the adaptive critic controller 
follows pilot input command of velocity where as the PINN controller is unable to maintain the commanded value. At velocity close to the stall velocity, it is observed that the PINN controller's response diverges with time. 


\section{CONCLUSION}

The objective of this thesis is to develop a neural network based adaptive controller for a general aviation aircraft. A systematic method is developed for designing the neural network based adaptive controller. Linear control theory is used to determine the architecture and the initial weights of the neural networks in what is called the initialization phase. The initialized neural networks meet performance specifications of the linear controller that they replace. In the next phase, referred to as online training phase, the neural networks are allowed to adapt online based on actual system dynamics as they arise. The online adaptation of the neural networks compensates for nonlinear dynamics as well as control failures and parameter variations. As demonstrated from the numerical results, the adaptive neural network based controller adapts to partial control failures as well as parameter variations while providing system response that is almost identical to the optimal system response. It is also apparent is that in the normal flight envelope for the Bonanza, the aircraft behavior is close to linear that the controller initialized to match the linear controller gains at the design points (PINN controller) is so robust, that even in the presence of modeling error and failures, it provides adequate handling qualities, and not much adaptation is required from the adaptive critic algorithm. In the non-linear regime of the Bonanza, at velocities close to stall, it is observed that the PINN controller becomes unstable whereas the adaptive critic algorithm adapts for the non-linearity and retains stability. Comparing the dynamic inverse method with the adaptive critic method of this thesis shows that the dynamic inverse method compensates faster for parameter variations and control failures than the adaptive critic methodology but the PINN controller of the adaptive critic method is much more robust in the presence of failures that the dynamic inverse method, both with adaptation turned off. This may impact which controller can be certified for general aviation aircraft. 
The main recommendation for future work is to evaluate the method proposed in this thesis to various other practical systems. Another important area of research could focus on using a different approach in the design of the linear controller. Since optimal estimation problem is seen as a dual of the optimal control problem, a logical step would be to expand the process proposed in this design to the development of a near optimal estimator. The initialization of the neural networks could be based on Kalman gains obtained at different operating points. Future work could also be focused on proofs for the stability and robustness of the neural network learning algorithm. 
REFERENCES 


\section{REFERENCES}

[1] Suresh, S, Omkar, S.N, Mani, V and Sundarajan, S, "Direct Adaptive Neural Flight Controller for F-8 Fighter Aircraft." Journal of guidance, control and dynamics Vol. 29, No. 2, pp. 454-464, March-April 2006.

[2] Kim, Byoung and Calise, Anthony, "Nonlinear Flight Control Using Neural Networks." Journal of guidance, control and dynamics, Vol. 20, No. 1, pp. 26-33, January-February 1997.

[3] Rysdyk, Rolf and Calise, Anthony, “Adaptive Model Inversion Flight Control for Tiltrotor Aircraft", American helicopter society annual forum, Washington D.C, May 1998.

[4] Perhinschi, M.G., Napolitano, M.R., Campa, G., Fravolird, M.L., Massotti, L., Lando, M. "Augmentation of a non linear dynamic inversion scheme within the NASA IFCS F-15 WVU simulator", American Control Conference, 2003. Proceedings of the 2003, Volume 2, Issue, June 4-6, 2003 Page(s): 1667 - 1672.

[5] Gundy-Burlet, Karen, Krishnakumar, K, Limes, Greg and Bryant, Don, "Augmentation of an Intelligent Flight Control System for a Simulated C-17 Aircraft.” Journal of Aerospace, Computing, Information and Communication, Vol. 1, pp. 526-542, December 2004.

[6] Steck, James E., Pesonen, Urpo J., Rokhsaz, Kamran, Bruner, Hugh Samuel and Duerksen, Noel, “Adaptive Neural Network Inverse Controller for General Aviation Safety.” Journal of guidance, control and dynamics, Vol. 27, No. 3, pp. 434-443, May-June 2004.

[7] Steck, James E., Pesonen, Urpo J., Rokhsaz, Kamran, Singh, Balbahadar and Chandramohan, Rajeev "Effect of Turbulence on an Adaptive Dynamic Inverse Flight Controller." Infotech@Aerospace,26 - 29 September 2005, AIAA-2005-7038. Arlington, Virginia

[8] Rokhsaz, Kamran, Steck, James E., Singh, Balbahadar and Chandramohan, Rajeev "Response of An Advanced Flight Control System to Microburst Encounters" 2005 Transactions Journal of Aerospace, SAE, pp.1432-1441.

[9] Kulkarni, Nilesh and Phan, Minh, "A Neural Network Based Design of Optimal Controllers for Nonlinear Systems." AIAA Guidance, Navigation, and Control Conference and Exhibit 5-8 AIAA-2002-46884, August 2002, Monterey, California.

[10] Balakrishnan, S.N and Biega, Victor, "Adaptive-Critic-Based Neural Networks for Aircraft Optimal Control." Journal of guidance, control and dynamics Vol. 19, NO. 4, pp. 893-898, JulyAugust 1996.

[11] Saini, G., Balakrishnan, S.N., Person, C., "Adaptive critic based neurocontroller for autolanding of aircraft with varying glideslopes." International conference on neural networks, Vol. 4, pp. 2288 - 2293, 1997. 
[12] Shin, Yoonghyun, Johnson, Matthew D., Calise, Anthony J, "Neural Network-Based Adaptive Control for Nonlinear Flight Regimes", AIAA Guidance, Navigation and Control Conference, AIAA-2003-5717, August 2003.

[13] Williams-Hayes, Peggy, "Flight Test Implementation of a Second Generation Intelligent Flight Control System”, infotech@Aerospace, AIAA-2005-6995, 26-29 September 2005, Arlington, Virginia.

[14] Ferrari, Silvia and Stengel, Robert “Online Adaptive Critic Flight Control.” Journal of guidance, control and dynamics, Vol. 27, No. 5, pp. 777-786, September-October 2004.

[15] Bellman, R. E., “Dynamic Programming”, Princeton University Press, Princeton, NJ, 1957.

[16] Ferrari, Silvia “Algebraic and Adaptive Learning in Neural Control Systems", Dissertation, Princeton, November 2002.

[17] Prokhorov, D. and Wunsch, D. “Adaptive Critic Designs," IEEE Trans. on Neural Networks, Vol. 8, No. 5, pp. 997-1007, 1997.

[18] Burl, Jeffrey B. "Linear Optimal Control $\mathrm{H}_{2}$ and $\mathrm{H} \infty$ Methods", Addison Wesley, 1999.

[19] Howard, R., "Dynamic Programming and Markov Processes”, MIT Press, Cambridge, MA, 1960.

[20] Ferrari, Silvia and Stengel, R.F. "Classical/Neural Synthesis of Nonlinear Control Systems", Journal of Guidance, Control, and Dynamics, Vol. 25, No. 3, May-June 2002.

[21] Ferrari, Silvia and Stengel, R.F. "Smooth Function Approximation using Neural Networks", IEEE Transactions on Neural Networks, Vol.16, No. 1, January 2005.

[22] Stengel, R. F., “Flight Dynamics”, Princeton University Press, 2004.

[23] Huang, C. and Stengel, R. F. "Restructurable Control Using Proportional-Integral Model Following," Journal of Guidance, Control, and Dynamics, Vol. 13, No. 2, pp. 303-309, 1990.

[24] Nelson, R. C., "Flight Stability and Automatic Control”, McGraw-Hill, Inc., New York, NY, 1989.

[25] Stengel, R. F., “A Unifying Framework for Longitudinal Flying Qualities Criteria,” Journal of Guidance, Control, and Dynamics, Vol. 6, No. 2, pp. 84-90, 1983.

[26] Roskam, Jan, “Airplane Flight Dynamics and Automatic Flight Controls.” DARcorporation, 2003. 